\title{
Flora of kurgans in the Pontic herb(-rich) grass steppe zone in Ukraine
}

\author{
IVAN IVANOVICH MOYSIYENKO \\ BARBARA SUDNIK-WÓJCIKOWSKA
}

МойсІєнКо І.І., СуднІк-ВойцИКОВСьКА Б., 2009: Флора курганів зони різнотравно- та багаторізнотравно-злакових степів в Україні. Чорноморськ. бот. ж., т. 5, N 3: 333 369.

Представлені результати дослідження флористичного багатства курганів в зоні різнотравно- та багаторізнотравно-злакових степів (згідно 3 MAP OF THE NATURAL VEGETATION OF EUROPE 2000: «the west and central Pontic herb-grass steppe» (M5) тa «west and central Pontic herb-rich grass steppe» (M1) зони) в Миколаївській та Кіровоградській областях. Вивчено 29 курганів понад 3 м. заввишки, що розташовані на території понад

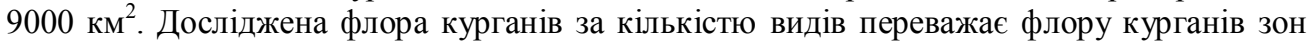
злакових та пустельних степів і налічує 439 видів. Кількість видів на одному кургані змінюється від 89 до 171 (середня 125,5). В зоні різнотравно- та багаторізнотравнозлакових степів переважають гемікриптофіти, на відміну від флор курганів зон, що розташовані південніше, де переважають терофіти. Також більш чисельними виявились тут фанерофіти (8,7 \%). Короткоживучі рослини (одно-, дво-, трирічні монокарпіки) складають 35,1 \% флори. Більшість 3 них є синантропними видами, переважно бур'яни 3 оточуючих полів. Загалом на курганах виявлено 113 видів адвентивних рослин. Археофіти та кенофіти складають 23,5 \% флори курганів.

В цілому на досліджених курганах було зафіксовано 19 синтаксонів вищого рангу. Види, що асоційовані з степовими синтаксонами: Festuco-Brometea, Festucetea vaginatae, Polygono-Artemisietea та Galietalia veri є найчисельнішими і складають 49,9 \% флори курганів. Як і у флорі курганів злакових та пустельних степів домінують види класів Festuco-Brometea та Stellarietea mediae, що підкреслює напівприродний характер флори курганів. Виявлено низку раритетних видів рослин: Adonis vernalis, Amygdalus nana, Anemone sylvestris, Astragalus dasyanthus, Crocus reticulatus, Elytrigia stipifolia, Galium volhynicum, Goniolimon tataricum, Hesperis tristis, Iris halophila, Limonium platyphyllum, Linaria biebersteinii, Ornithogalum kochii, Phlomis hybrida, Stipa capillata, S. lessingiana. Кургани зони різнотравно- та багаторізнотравно-злакових степів, як і інших зон виступають рефугіумами степової флори. Вони досить рівномірно розташовані в степовій зоні і відіграють важливу роль в локальному відновлені природної рослинності, тому повинні охоронятися не лише як археологічні, а і як природні пам'ятки.

Ключові слова: кургани, рефугіум степової флори, флористичне різноманіття, Понтичний різнотравно-злаковий та багаторізнотравно-злаковий степ, охорона курганів, південь Украӥни.

Moysiyenko I.I., SUDNiK-WÓJCIKOWSKA B., 2009: Flora of kurgans in the Pontic herb(-rich) grass steppe zone in Ukraine. Chornomorsk. bot. z., vol. 5, N 3: 333-369.

The results of study of floristic diversity of kurgans in the Pontic herb(-rich) grass steppe zone (according to MAP OF THE NATURAL VEGETATION OF EUROPE 2000: the west and central Pontic herb-grass steppe (M5) and west and central Pontic herb-rich grass steppe (M1) zones) in Mykolaiv and Kirovograd Regions, are presented. Twenty-nine kurgans higher than $3 \mathrm{~m}$, distributed over an area approx. $9000 \mathrm{~km}^{2}$, were surveyed. The investigated kurgan flora contains more species than the flora of the barrows in the west Pontic grass steppe and desert steppe zones, being estimated at 439 species. The number of species on a single kurgan varied from 89 to 171 with 125,5 on average. Hemicryptophytes dominated in the kurgan flora $(38,8 \%)$ of the Pontic herb(-rich) grass steppe zone, whereas therophytes prevailed in barrow flora in the steppe zones southwards. Phanerophytes were more numerous as well $(8,7 \%)$.

(C) I.I. Мойсієнко, Б. Суднік-Войциковська

Чорноморськ. бот. ж., 2009, т. 5, N3: 333-369. 
(Annuals and biennials constituted 35,1\% of the kurgan flora. Most of them are synanthropic species, e.g. weeds derived from the surrounding fields. The total number of alien species (anthropophytes) reached 113. Archaeophytes and kenophytes comprised 23,5\% of the kurgan flora.

A total of 19 syntaxa of higher rank were represented in all the kurgans studied. Species associated with steppe syntaxa such as Festuco-Brometea, Festucetea vaginatae, PolygonoArtemisietea and Galietalia veri comprised as much as 49,9\% of the kurgan flora. As in the case of the desert and grass steppes, species belonging to classes Festuco-Brometea and Stellarietea mediae were predominant in the herb rich grass steppe, which also confirmed the semi-natural character of the kurgan flora. The species of particularly high floristic value were: Adonis vernalis, Amygdalus nana, Anemone sylvestris, Astragalus dasyanthus, Crocus reticulatus, Elytrigia stipifolia, Galium volhynicum, Goniolimon tataricum, Hesperis tristis, Iris halophila, Limonium platyphyllum, Linaria biebersteinii, Ornithogalum kochii, Phlomis hybrida, Stipa capillata, S. lessingiana. The investigations carried out in the Pontic herb(-rich) grass steppe zone confirm that kurgans constitute refugia for the steppe flora. They are more or less uniformly distributed in the steppe zone, and could play an important role in the local restoration of the natural plant cover. Therefore, they should be put under protection as archeological and nature monuments.

Key words: barrows, refugia of steppe flora, floristic diversity, herb-grass steppe, herb-rich grass steppe, protection of kurgans, the South of Ukraine.

МоЙСИЕНКО И.И., СудНИК-ВОЙЦИКОВСКАЯ Б., 2009: Флора курганов зоны разнотравно- и богаторазнотравно-злаковых степей в Украине. Черноморск. бот. ж., т. 5, N 3:333-369.

Представлены результаты исследования флористического богатства курганов в зоне разнотравно- и богаторазнотравно-злаковых степей (согласно с MAP OF THE NATURAL VEGETATION OF EUROPE 2000: «the west and central Pontic herb-grass steppe» (M5) и «west and central Pontic herb-rich grass steppe» (M1) зоны) в Николаевской и Кировоградской областях. Изучено 29 курганов более 3 м. высотой, которые расположены на территории свыше 9000 км2. Исследованная флора курганов по количеству видов преобладает над флорой курганов зоны злаковых и пустынных степей и насчитывает 439 видов. Количество видов на одном кургане изменяется от 89 до 171 (в среднем 125,5). В зоне разнотравно- и богаторазнотравно-злаковых степей преобладают гемикриптофиты, в отличии от флор курганов зон расположенных южнее, где преобладают терофиты. Также более многочисленными оказались тут фанерофиты $(8,7 \%)$. Краткоживущие растения (одно-, дво-, многолетние монокарпики) составляют 35,1 \% флоры. Большинство из них являются синантропными видами, преймущественно сорняками с прилегающих полей. Всего на курганах выявлено 113 видов адвентивных растений. Археофиты и кенофиты составляють 23,5 \% флоры курганов.

В целом на исследованных курганах было зафиксировано растения 19 синтаксонов высшего ранга. Виды ассоциированые со степными синтаксонами: Festuco-Brometea, Festucetea vaginatae, Polygono-Artemisietea u Galietalia veri являются наиболее многочисленными и составляют 49,9 \% флоры курганов. Как и во флоре курганов злаковых и пустынных степей домінируют виды класов Festuco-Brometea и Stellarietea mediae, что подчеркивает полуестественный характер флоры курганов. Выявлено ряд созофитов: Adonis vernalis, Amygdalus nana, Anemone sylvestris, Astragalus dasyanthus, Crocus reticulatus, Elytrigia stipifolia, Galium volhynicum, Goniolimon tataricum, Hesperis tristis, Iris halophila, Limonium platyphyllum, Linaria biebersteinii, Ornithogalum kochii, Phlomis hybrida, Stipa capillata, S. lessingiana. Курганы зоны разнотравно- и богаторазнотравно-злаковых степей, как и других зон выступают рефугиумами степной флоры. Они достаточно равномерно расположены в степной зоне и играют важную роль в локальном востановлении природной растительности, поэтому, должны охранятся не только как археологические памятники, но и как природные.

Ключевые слова: курганы, рефугиум степной флоры, флористическое разнообразие, Понтическая разнотравно-злаковая и богаторазнотравно-злаковая степь, охрана курганов, юг Украины. 


\section{Introduction}

The present work continues the series of publications dealing with the biodiversity of flora of kurgans in the steppe zone of southern Ukraine. Earlier papers [MOYSIYENKO, SUDNIKWÓJCIKOWSKA 2006A, 2006B, 2006C, SUDNIK-WÓJCIKOWSKA, MOYSIYENKO 2006, 2008] were concerned with the flora of kurgans located southwards: desert (west and central Pontic) steppe zone and grass steppe zone - ,bednoye raznotravie” (west Pontic grass steppe zone).

The aim of this study was to assess the richness and specific character of the flora of kurgans within the steppe zone which is commonly referred to as the grass steppe zone „bogatoye razotravie”. Due to its high quality soils the above mentioned area has been utilized agriculturally for a long time. The steppe grasslands have survived only as small fragments, e.g. in balkas, river valleys and in nature reserves. Within the investigated area there are some valuable reserves, such as „Jelaniecki Step” and „Granitove Pobuzhzhya”. As in the case of the other zones, the kurgans in the above mentioned zone are an interesting habitat in terms of the steppe flora.

\section{Study area}

The area surveyed is located in the southern part of Dniepr Highland, in the Mykolaiv and Kirovograd Regions. The investigated kurgans are distributed over an area of about $9000 \mathrm{~km}^{2}$ in the northern part of the proper grass steppe zone. Traditionally, the true (proper) grass steppe zone is divided into two subzones: grass and herb-grass steppes (in ukr./russ.: підзони типчаково-ковилових/злаковых та різнотравно-типчаково-ковилових/разнотравно- и богаторазнотравнозлаковых степей [БІЛИК, 1973, ЛАВРЕНКО и др., 1991]). According to the nomenclature proposed by BOHN et al. [2000] (Map of the natural vegetation of Europe) the true grass steppe zone is divided into two parts: the southernmost west Pontic grass steppe zone (M12), and the northern "bogatoye raznotravie" which is divided into two subzones: west and central Pontic herb-grass steppe (zone M5) and west and central Pontic herb-rich grass steppe (zone M1). In the present work we adopted the traditional classification system of steppes. The above two subzones were treated as one zone which we referred to as the Pontic herb(-rich) grass steppe zone. In Europe it stretches as a narrow strip from south-west to north-east. The zone extends from Romania (Dobruja) and Moldova through Podolian Highland, up to Dnieper and Azovian Highland, Donez hilly country and lower courses of the Don river to the southern part of Ural. In Asia this steppe lies along the parallel of latitude extending from the southern part of West Siberia to Kazakhstan and Altai. It borders the forest-steppe zone on the north [ЛАВРЕНКО и др., 1991].

The landscape is dominated by a plateau (usually not higher than $200 \mathrm{~m}$ a.s.l.) which is crisscrossed by numerous valleys and ravines. The area is distinguished by low elevation topography, usually up to 300-500 m a.s.l. (e.g. Donetz Hilly Country, Central Russian Highlands, Volgian and Stavropol' Highlands). Eolian and fluvial sediments dominate with outcrops of crystalline rocks, sandstone or limestone (Cretaceous sediments in the basement). The soils are mainly moderately dry, meso- or mesoeutrophic, humic chernozem and chernozem variants with a thick humus layer. The area includes typical steppes located in watersheds with well-developed as well as their edaphic variants: petrophytic and psammophytic steppes. The petrophytic steppes occur along river banks and balkas, in the outcrops of crystalline rocks (e.g. granite, gneiss) or sedimentary rocks (e.g. limestone, chalk, marl, sandstone). The psammophytic steppe is associated with sandy river terraces. However, saline soils are less frequently encountered. Solonchak- and solonetz soils occur locally on river terraces [МАРИНич и др., 1985; ПРИРОДА УКРАИНСКОЙ ССР. ПОЧВЫ, 1986]. 
The zone is characterized by moderately continental and adequately dry climate, with eastern dry winds and frequent droughts. The climate becomes more continental in type from west to east. The average annual temperature ranges from $10-12^{\circ} \mathrm{C}$ in the west to $7-8^{0} \mathrm{C}$ in the east. The average temperature of the warmest month (July) is $20-23^{\circ} \mathrm{C}$ (absolute maximum: 40 ${ }^{0} \mathrm{C}$ ). The average temperature of the coolest month (January) is $-9--4^{0} \mathrm{C}$ (absolute minimum: -38 ${ }^{0} \mathrm{C}$ ). The average annual precipitation varies between 350 and $520 \mathrm{~mm}$, local rainfall is higher, e.g. in Donetz Hilly - $540 \mathrm{~mm}$, and in Stavropol' Highlands - even more than $800 \mathrm{~mm}$. Northeastern winds prevail. In summer the area is often characterized by periods of drought and „sukhoviey” winds prevail. Occasionally dust storms are noted [МАРИНИЧ и др., 1985. ПРИРОДА УКРАИНСКОЙ ССР. КЛИМАТ, 1984].

The herb(-rich) grass steppe zone is characterized by the presence of forests in balkas. The southern border of the zone is marked by forests of the above type. These types of forests are not found further south (in the southern part of grass steppe zone). In the zone located north of the herb(-rich) grass steppe zone, the forests occur not only in balkas but also in flat, open areas ("plakorie").

The Pontic herb(-rich) grass steppe zone can be distinguished from the other vegetation types occurring southwards by a smaller contribution of tuft grasses and, therefore, higher representation of perennial dicotyledonous plants and shrub communities. A higher number of species and increased biomass per unit area are observed. However, ephemerals and ephemeroids play a less significant role here. The moss and lichen layer is usually poorly developed.

A total of 29 kurgans (R1-R29) were investigated: 16 (R1-R15) were located in the west and central Pontic herb-grass steppe zone (M5) and 13 (R16-R18-29) in the west and central Pontic herb-rich grass steppe zone (M1); Fig. 1. Due to problems in establishing the location of the kurgans on archaeological and physical-geographical maps, GPS was used to locate the barrows (Table 1).

\section{Material and methods}

In the present work the same methods as those used in an earlier study [MOYSIYENKO, SUDNIK-WÓJCIKOWSKA 2006A, 2006B] were applied. The following criteria were used to select the 29 kurgans:

- kurgans more than $3 \mathrm{~m}$ high were chosen;

- good state of preservation of kurgans;

- the state of preservation of the plant cover; it was assumed that the presence of typical steppe species, such tuft grasses as Festuca valesiaca, Koeleria cristata and Stipa capillata, was indicative of a relatively good condition of plant cover.

The flora of 5 microhabitats within 29 kurgans was investigated. The data were compiled in a table (Appendix 1) which contained the following additional information about each taxon: its occurrence and abundance in particular microhabitats within the kurgans investigated, species life form, its status in the historical-geographical classification, and origin in the case of alien species. Floristic analysis was conducted and the specific character of the kurgan flora within the Pontic herb(-rich) grass steppe zone was determined. A five-grade scale was used to assess the frequency category of the species (see Fig. 3). Special attention was paid to the proportion of short living plants and alien species in the kurgan flora.

The species nomenclature follows S. MOSYAKIN, M. FEDORONCHUK [1999], Latin names of syntaxa are given according to В. СоЛОМАХА [1996], Б. МІРКІн, Л. НАУмОВА [1998], and W. MATUSZKIEWICZ [2001]. 


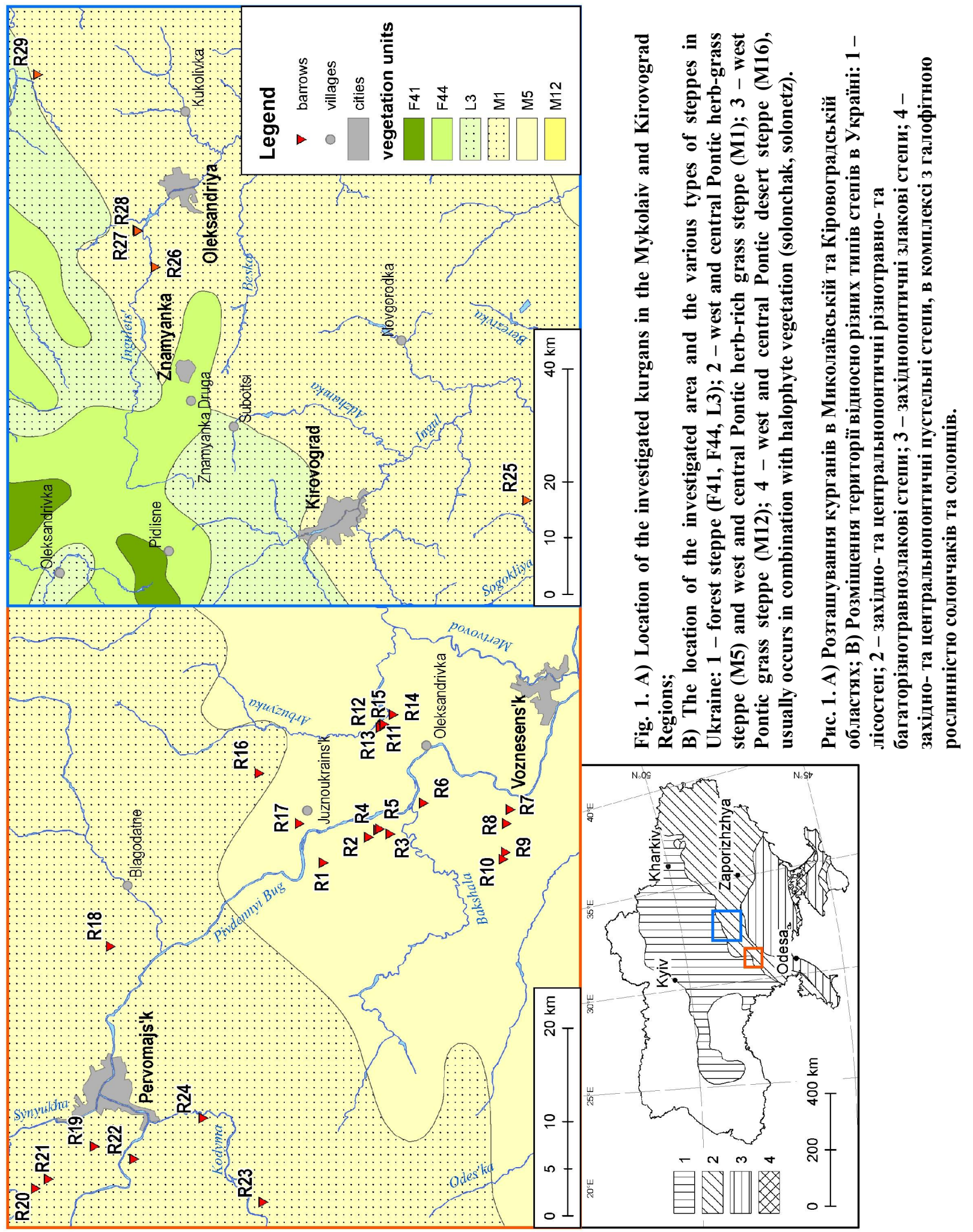


Moysiyenko I.I., Sudnik-Wójcikowska B.

Table 1

The location and size of the investigated kurgans in the Pontic herb(-rich) grass steppe zone in Mykolaiv and Kirovograd Region

Таблиця 1

Локалізація та розміри досліджених курганів в зоні понтичного різнотравно- та багаторізнотравнозлакового степу на території Миколаївської та Кіровоградської областей

\begin{tabular}{|c|c|c|c|c|c|}
\hline $\begin{array}{c}\text { Code of } \\
\text { the kurgan }\end{array}$ & $\begin{array}{c}\text { Location } \\
\text { (nearest village) }\end{array}$ & $\begin{array}{l}\text { Longitude } \\
\text { (E) }\end{array}$ & $\begin{array}{l}\text { Latitude } \\
\text { (N) }\end{array}$ & $\begin{array}{l}\text { Height of } \\
\text { kurgan } \\
\text { (m) }\end{array}$ & $\begin{array}{c}\text { Diameter } \\
\text { of kurgan } \\
\text { (m) }\end{array}$ \\
\hline \multicolumn{6}{|c|}{ Region Mykolaiv } \\
\hline \multicolumn{6}{|c|}{ District Domanivka } \\
\hline R1 & Bogdanivka & $31^{0} 07^{\prime} 51.9^{\prime \prime}$ & $47^{0} 48^{\prime} 15.1^{\prime \prime}$ & 7 & 80 \\
\hline $\mathrm{R} 2$ & Bogdanivka -Wynogradnyi Sad & $31^{0} 09^{\prime} 27.0^{\prime \prime}$ & $47^{0} 45^{\prime} 27.0^{\prime \prime}$ & 6,5 & 80 \\
\hline R3 & Wynogradnyi Sad & $31^{0} 09 ' 26.9^{\prime \prime}$ & $47^{0} 44^{\prime} 12.0^{\prime \prime}$ & 4,5 & 50 \\
\hline $\mathrm{R} 4$ & Wynogradnyi Sad & $31^{0} 09^{\prime} 55.5^{\prime \prime}$ & $47^{0} 44^{\prime} 51.5^{\prime \prime}$ & 4 & 50 \\
\hline R5 & Wynogradnyi Sad & $31^{0} 10^{\prime} 01.1^{\prime \prime}$ & $47^{0} 44^{\prime} 44.9^{\prime \prime}$ & 3 & 40 \\
\hline R6 & Prybuzhzhya & $31^{0} 11^{\prime} 37.4^{\prime \prime}$ & $47^{0} 42^{\prime} 02.2^{\prime \prime}$ & 5 & 65 \\
\hline R7 & Zhovtneve - Zabara & $31^{\circ} 09^{\prime} 59.4^{\prime \prime}$ & $47^{0} 37^{\prime} 08.8^{\prime \prime}$ & 6 & 80 \\
\hline $\mathrm{R} 8$ & Zhovtneve - Zabara & $31^{\circ} 08^{\prime} 51.5^{\prime \prime}$ & $47^{0} 37^{\prime} 28.5^{\prime \prime}$ & 6 & 70 \\
\hline R9 & Zhovtneve - Zabara & $31^{\circ} 06^{\prime} 29.5^{\prime \prime}$ & $47^{0} 37^{\prime} 47.4^{\prime \prime}$ & 7,5 & 80 \\
\hline $\mathrm{R} 10$ & Zhovtneve - Zabara & $31^{0} 05^{\prime} 55.3^{\prime \prime}$ & $47^{0} 37^{\prime} 58.8^{\prime \prime}$ & 6,5 & 80 \\
\hline \multicolumn{6}{|c|}{ District Voznesens'k } \\
\hline R11 & Trykraty & $31^{0} 18 ’ 38.3^{\prime \prime}$ & $47^{0} 43^{\prime} 39.7^{\prime \prime}$ & 5 & 70 \\
\hline $\mathrm{R} 12$ & Trykraty & $31^{0} 18^{\prime} 36.7^{\prime \prime}$ & $47^{0} 43^{\prime} 43.7^{\prime \prime}$ & 7 & 80 \\
\hline R13 & Trykraty & $31^{0} 18^{\prime} 30.3^{\prime \prime}$ & $47^{0} 43^{\prime} 49.3^{\prime \prime}$ & 7 & 50 \\
\hline R14 & Trykraty & $31^{0} 19^{\prime} 25.6^{\prime \prime}$ & $47^{0} 42^{\prime} 59.0^{\prime \prime}$ & 7 & 80 \\
\hline R15 & Trykraty & $31^{0} 18^{\prime} 43.2^{\prime \prime}$ & $47^{0} 43^{\prime} 36.0^{\prime \prime}$ & 4 & 50 \\
\hline \multicolumn{6}{|c|}{ District Arbuzynka } \\
\hline R16 & Arbuzynka & $31^{0} 16^{\prime} 11.5^{\prime \prime}$ & $47^{0} 51^{\prime} 06.9^{\prime \prime}$ & 5 & 70 \\
\hline R17 & Yuzhnoukrains`k & $31^{0} 11^{\prime} 27.1^{\prime \prime}$ & $47^{0} 49^{\prime} 15.6^{\prime \prime}$ & 5 & 70 \\
\hline \multicolumn{6}{|c|}{ District Pervomays'k } \\
\hline $\mathrm{R} 18$ & Blagodatne - Mygiya & $31^{\circ} 03^{\prime} 24.3^{\prime \prime}$ & $48^{0} 01$ '04.9' & 8 & 80 \\
\hline R19 & Chausove & $30^{\circ} 46^{\prime} 35.7^{\prime \prime}$ & $48^{0} 03^{\prime} 42.2^{\prime \prime}$ & 6 & 100 \\
\hline R20 & Lukashivka & $30^{\circ} 43^{\prime} 46.9 \prime$ & $48^{\circ} 07^{\prime} 23.9^{\prime \prime}$ & 8 & 90 \\
\hline $\mathrm{R} 21$ & Lukashivka & $30^{\circ} 44^{\prime} 23.6^{\prime \prime}$ & $48^{0} 06^{\prime} 35.6^{\prime \prime}$ & 5 & 40 \\
\hline R22 & Konets'pol' & $30^{0} 45^{\prime} 04.2^{\prime \prime}$ & $48^{0} 01^{\prime} 32.8^{\prime \prime}$ & 4,5 & 45 \\
\hline R23 & Kumari & $30^{0} 39^{\prime} 52.7^{\prime \prime}$ & $47^{0} 54^{\prime} 33.0^{\prime \prime}$ & 8 & 90 \\
\hline R24 & Kam'yanyi mist & $30^{0} 47^{\prime} 41.8^{\prime \prime}$ & $47^{0} 57^{\prime} 15.7^{\prime \prime}$ & 4,5 & 50 \\
\hline \multicolumn{6}{|c|}{ Region Kirovograd } \\
\hline \multicolumn{6}{|c|}{ District Kompaniivka } \\
\hline R25 & Zhyvanivka & $32^{0} 13^{\prime} 49.3$ & $48^{0} 12^{\prime} 33.1$ & 5 & 70 \\
\hline \multicolumn{6}{|c|}{ District Oleksandriya } \\
\hline R26 & Bandurivka & $32^{0} 55^{\prime} 52.7^{\prime \prime}$ & $48^{0} 43^{\prime} 53.0^{\prime \prime}$ & 3 & 40 \\
\hline R27 & Protopopivka & $33^{0} 01^{\prime} 36.8^{\prime \prime}$ & $48^{0} 44^{\prime} 57.7^{\prime \prime}$ & 4,5 & 60 \\
\hline R28 & Protopopivka & $33^{0} 0126.0^{\prime \prime}$ & $48^{0} 44^{\prime} 55.9^{\prime \prime}$ & 5 & 65 \\
\hline \multicolumn{6}{|c|}{ District Onufriivka } \\
\hline R29 & Onufriivka & $33^{0} 26^{\prime} 25.1^{\prime \prime}$ & $48^{0} 51^{\prime} 41.4^{\prime \prime}$ & 6,5 & 70 \\
\hline
\end{tabular}




\section{Biodiversity of the kurgan flora}

\section{Results}

A total of 439 species of vascular plants were reported from 29 kurgans in the Pontic herb(-rich) grass steppe zone (Appendix 1; the 3 additional species at the bottom of the table were found on the kurgans in this zone which were not investigated in the present study). The number of species on particular kurgans ranged from 89 to $171,125,5$ on average. The kurgan flora in the grass steppe was richer in species than the flora of the barrows in the desert steppe zone (305 species, MOYSIYENKO, SUDNIK-WÓJCIKOWSKA 2006C) and in the west Pontic grass steppe zone (355, SUDNIK-WÓJCIKOWSKA, MOYSIYENKO 2006). The species belonged to 248 genera and 53 families (in the grass steppe: 209 and 51, respectively and in the desert steppe: 192 and 48, respectively). The following families were represented by the greatest number of taxa: Asteraceae, Poaceae, Fabaceae, Lamiaceae, Brassicaceae, Rosaceae, Scrophulariaceae, Caryophyllaceae, Apiaceae, Ranunculaceae, Liliaceae, Polygonaceae, Boraginaceae, Chenopodiaceae and Rubiaceae (Fig. 2). When comparing with the flora of kurgans located in the other zones, the role of the following plant families increased: Lamiaceae, Rosaceae, Apiaceae. Genera represented by the highest number of taxa were, as follows: Veronica (10), Artemisia (7), Astragalus (7), Achillea (6), Euphorbia (6), Galium (6), Medicago (6), Verbascum (6), Allium (5), Gagea (5), Potentilla (5) and Vicia (5).

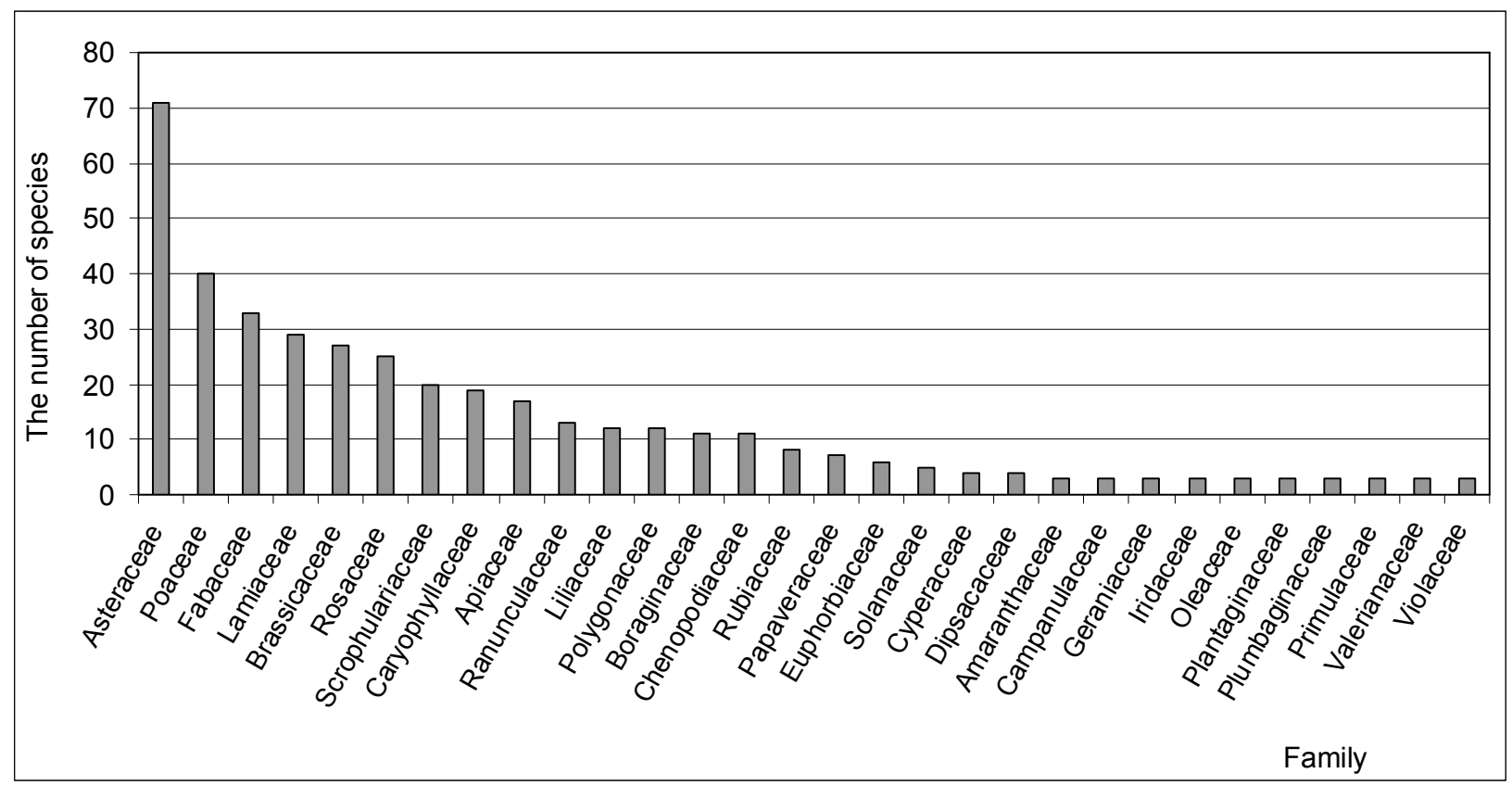

Fig. 2. The most important families (in terms of species number) in the total flora of kurgans in the Pontic herb(-rich) grass steppe zone.

Рис. 2. Найбільш представлені (за кількістю видів) у флорі курганів зони понтичного різнотравнота багаторізнотравно-злакового степу родини.

About 156 species (36\% of the total kurgan flora) with the first (I) frequency class (Fig. 3) were considered sporadic or accidental (on 1-2 kurgans only). The group of common species recorded on 23-29 of the studied kurgans (frequency class V) made up about 10\% of the flora: Artemisia absinthium (29), Artemisia austriaca (29), Convolvulus arvensis (29), Elytrigia repens (29), Linaria biebersteinii (29), Melandrium album (29), Poa angustifolia (29), Salvia nemorosa (29), Festuca valesiaca (29), Lactuca serriola (29), Berteroa incana (28), Carduus acanthoides (28), Coronilla varia (28), Euphorbia virgata (28), Falcaria vulgaris (28), Achillea setacea (27), Consolida paniculata (27), Koeleria cristata (27), Lamium amplexicaule (27), Sisymbrium loeselii (27), Stipa capillata (27), Hyoscyamus niger (26), Lathyrus tuberosus (26), Medicago falcata (26), Verbascum lychnitis (26), Agropyron 
pectinatum (25), Ballota nigra (25), Chenopodium album (25), Centaurea adpressa (25), Euphorbia agraria (25), Galium aparine (25), Potentilla argentea (25), Senecio erucifolius (25), Achillea stepposa (24), Euphorbia stepposa (24), Onopordum acanthium (24), Stachys recta (24), Buglossoides arvensis (23), Cirsium arvense (23), Hypericum elegans (23), Melilotus officinalis (23), Seseli tortuosum (23), Viola kitaibeliana (23).

The total abundance of every species within the kurgans theoretically ranged from 0 up to 435 (a 3-grade scale was used to estimate the abundance of species, and 5 microhabitats within the 29 kurgans studied were taken into account: $3 \times 5 \times 29=435)$. The abundance of only a few species exceeded 200, i.e.: Poa angustifolia (289), Elytrigia repens (258), Salvia nemorosa (256), Convolvulus arvensis (249), Falcaria vulgaris (241), Festuca valesiaca (233), Coronilla varia (215), Agropyron pectinatum (209) and Artemisia austriaca (204). At the same time, the above taxa were the most frequently encountered species.

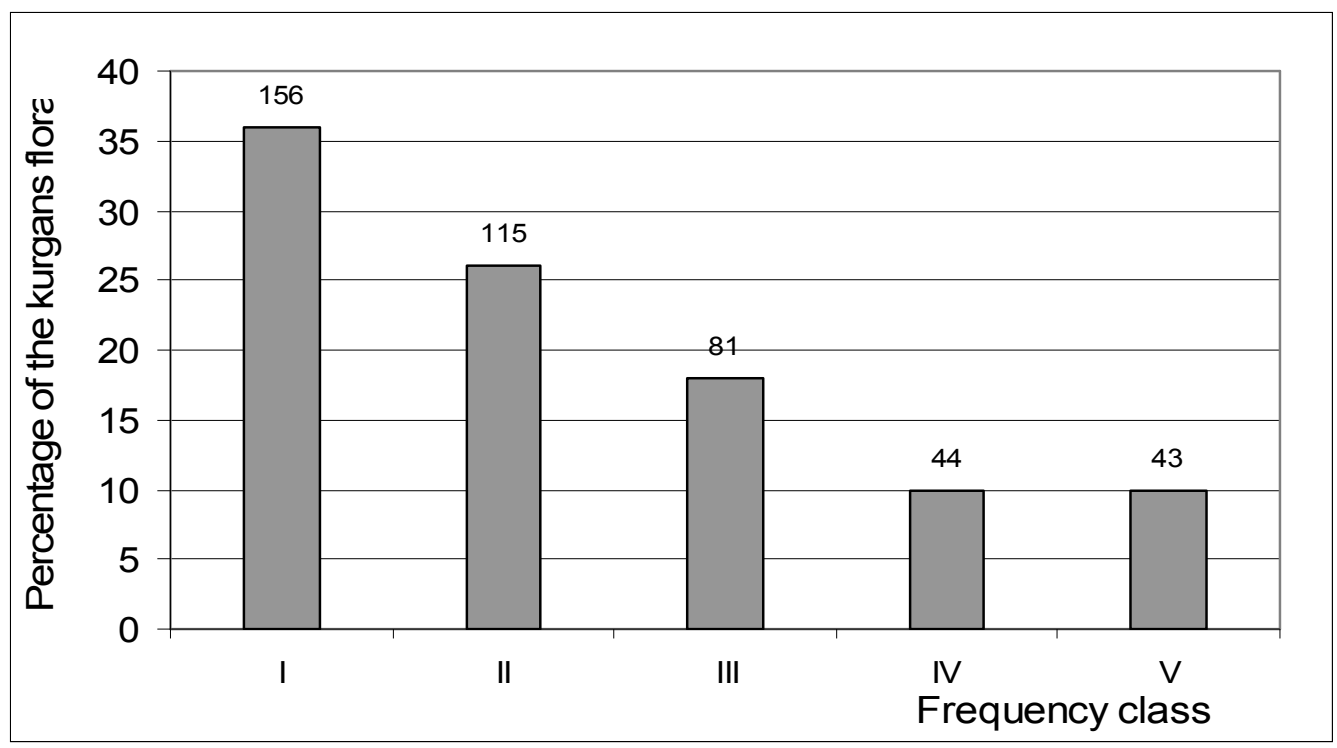

Fig. 3. Subdivision of the flora of kurgans in the Pontic herb(-rich) grass steppe zone by frequency class (the total number of species in each category is indicated at the top of the bar). Frequency classes: see Material and Methods.

Рис. 3. Розподіл флори курганів зони понтичного різнотравно- та багаторізнотравно-злакового степу за класами частоти трапляння (на верхівці стовпчика вказана абсолютна кількість видів у кожній категорії). Класи частоти трапляння: дивись Матеріали та методи.

\section{Spectrum of life forms}

The spectrum of life forms in the flora of kurgans in the Pontic herb(-rich) grass steppe zone corresponded to that of the flora of the proper steppe zone. However, hemicryptofites (no therophytes) are dominating group of species (38,8\%). Perennial herbaceous caudex plants with a somewhat woody lower part of the stem seem better adapted to the conditions prevailing in the steppe, such as water deficit stress. Sod grass plants, which dominate in the steppe phytocoenoses, were qualified as hemicryptophytes: Agropyron pectinatum, Festuca rupicola, Festuca valesiaca, Koeleria cristata, Stipa capillata, Stipa lessingiana as well as a number of herbaceous dicotyledonous plants: Asperula montana, Astragalus asper, Astragalus austriacus, Campanula sibirica, Centaurea stereophylla, Cephalaria uralensis, Dianthus guttatus, Eremogone longifolia, Euphorbia leptocaula, Ferulago galbanifera, Galatella villosa, Goniolimon besserianum, Inula oculus-christi, Jurinea multiflora, Limonium tomentellum subsp. alutaceum, Nepeta parviflora, Pastinaca clusii, Potentilla astracanica, Salvia austriaca. The second largest group belonged to therophytes $(35,1 \%)$, which usually dominated in the west Pontic grass steppe zone $(43,2 \%)$, 
as well as in the desert steppe zone (46,5\%). Their high proportion in the flora of kurgans was determined by various anthropogenic factors. Unlike in the case of perennial species, anthropophytes dominated among therophytes $(55 \%)$. Other life forms played a smaller role in the flora of the barrows: geophytes comprised $14 \%$ of the kurgan flora, and chamaephytes $-3,4 \%$.

In the desert steppe zone phanerophytes comprised only $4 \%$ of the flora of kurgans (in the west Pontic grass steppe zone: $4,8 \%$ ). Their role in the flora of the barrows increased towards the north $(8,7 \%)$. In the Pontic herb(-rich) grass steppe zone the following species of native phanerophytes were recorded (being absent on the kurgans investigated in the zones southwards): Acer tataricum, Caragana frutex, Chamaecytisus austriacus, Euonymus europaea, Ligustrum vulgare, Rosa villosa, Rubus caesius, Solanum dulcamara, Swida sanguinea, Tilia europaea, Ulmus minor. In the case of the latter zone alien phanerophytes were: Acer negundo, Amelanchier lamarckii, Cerasus vulgaris, Colutea arborescens, Fraxinus pennsylvanica, Lonicera tatarica, Prunus divaricata, P. domestica, P. cerasifera.

The life form spectra differed in the particular microhabitats. Hemicryptophytes, which dominate in the flora of the steppe, attained their optimum development on the slopes, particularly on the southern side of the kurgans. As stated earlier, the slopes provided more favourable conditions for a number of steppe species. Compared with the top, they were less disturbed and were generally characterized by drier conditions than the foot of the kurgans. Species which were less tolerant of dry conditions were mainly encountered on the north slopes. Therophytes, among which were numerous anthropophytes, were mostly represented on the top of the barrows. Phanerophytes, which have a higher demand for moisture were more often found at the foot. The role of chamaephytes increased towards the top of the kurgans.

Considerable differences between the life form spectra of the particular microhabitats were detected. The top of the kurgans supported a higher number of therophytes and chamaephytes. Phanerophytes were associated mainly with the foot of the barrows. Geophytes were most abundant at the foot as well. The distribution of particular life forms on the kurgans was also determined by the exposition of the slope. Hemicryptophytes and therophytes were more often found on the southern side of the kurgans whereas the representation of phanerophytes and chamaephytes was higher on the northern side.

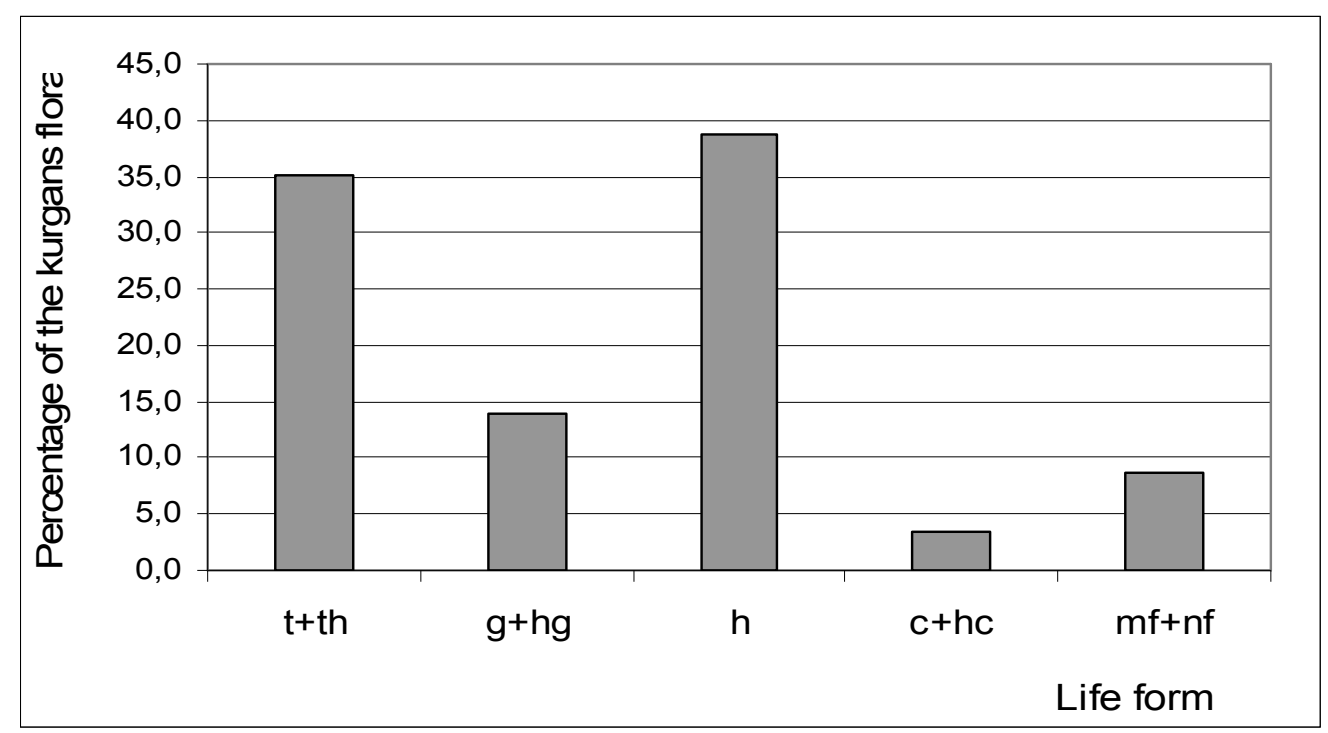

Fig. 4. Spectrum of life forms in the flora of kurgans in the Pontic herb(-rich) grass steppe zone (for abbreviations see Appendix 1).

Рис. 4. Спектр життєвих форм флори курганів зони понтичного різнотравно- та багаторізнотравнозлакового степу (прийняті скорочення дивись в Додатку 1). 


\section{Spectrum of socio-ecological groups}

The flora of kurgans was distinguished by a wide sociological range. It included the representatives of at least 19 syntaxa of higher ranks (Fig. 5). As in the case of kurgans in the desert and grass steppe zones, species representing communities of the Festuco-Brometea and Stellarietea mediae classes had the biggest share concerning complex groups of steppe grasslands and synanthropic communities, 57,2 \%, (i.e. 37,6\%, and 19,6\% respectively). Such domination reflects the character of the kurgan flora.

Species associated with steppe syntaxa: Festuco-Brometea, Festucetea vaginatae, Polygono-Artemisietea and Galietalia veri comprised as much as 49,9\% of the kurgan flora $(37,6 \% ; 4,6 \% ; 1,8 \% ; 5,9 \%$ respectively). Species representing synanthropic syntaxa (with the exception of Stellarietea mediae), such as classes: Artemisietea vulgaris and Agropyretea intermedio-repentis as well as the Plantaginetalia majoris order $(8,7 \%, 1,8 \%, 0,7 \%$ respectively) constituted $30,8 \%$ of the kurgan flora.

Under more favourable soil moisture conditions, species belonging to the classes Molinio-Arrhenatheretea (except Galietalia veri) and Bidentetea were found growing at the foot of the kurgans, mostly on their northern side. However, their proportion in the flora of kurgans was estimated at $4,8 \%$ and $0,005 \%$ respectively.

Species associated with forest and scrub communities were much better represented in the flora of kurgans in the Pontic herb(-rich) grass steppe zone than in the desert steppe and grass steppe zones. A higher number of syntaxa which were represented by a big number of species were recorded. A total of 5 classes of tree and shrub communities were represented on the kurgans. Species associated with the above mentioned syntaxa constituted $8,9 \%$ of the flora of kurgans. The plant communites from the above classes were usually found at the foot of the kurgans. Other syntaxa of higher ranks, including halophyte communities (Asteretea tripolii, Festuco-Puccinellietea) were poorly represented on the kurgans.

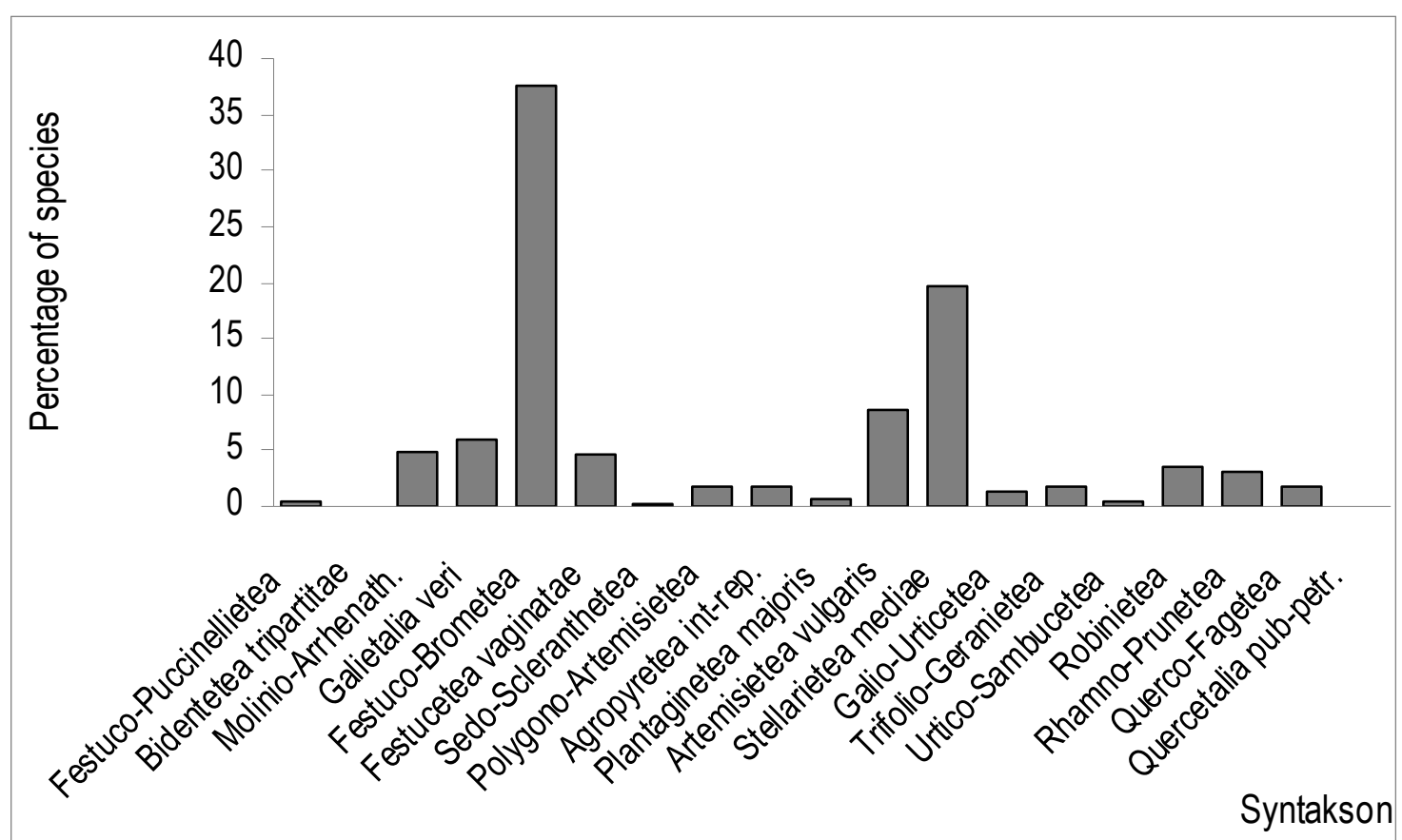

Fig. 5. The number of species of the syntaxa represented in the kurgan flora in the Pontic herb(-rich) grass steppe zone (the order of syntaxa is not random - syntaxa given in a gradient from natural to synanthropic).

Рис. 5. Кількість видів в синтаксонах представлених на курганах в зоні понтичного різнотравно- та багаторізнотравно-злакового степу (порядок синтаксонів не випадковий - в градіснті від натуральних до синантропних). 
4. Spectrum of species groups in the historical-geographical classification of plants

The spectrum of synanthropic species groups in the flora of kurgans in the Pontic herb(-rich) grass steppe zone (Tab. 2, Fig. 6) corresponded basically with the spectra for the desert and grass steppe zones.

Native species accounted for $74,2 \%$ of the flora of kurgans in investigated zone whereas non-synanthropic plants comprised $41,4 \%$ of the kurgan flora, the most frequently occurring species were: Achillea stepposa, Centaurea adpressa, Euphorbia stepposa, Festuca valesiaca, Hypericum elegans, Koeleria cristata, Salvia nemorosa, Seseli tortuosum, Stachys recta, Stipa capillata. The percentage of native and non-synanthropic species in the flora of kurgans was similar in the case of the desert steppe zone: $77,2 \%$ and $40,9 \%$, respectively and the west Pontic grass steppe zone: 70,8 \% and 39,5\%, respectively.

Depending on the level of transformation of the microhabitats into which they penetrate, apophytes can be subdivided into 2 groups: hemiapophytes $(21,4 \%$ of the total flora) and euapophytes $(11,4 \%$ ) (Fig. 6). With respect to frequency, hemiapophytes distinctly predominated over euapophytes, e.g. 14 hemiapophytes belonged to frequency class $\mathrm{V}$ (Achillea setacea, Agropyron pectinatum, Artemisia austriaca, Coronilla varia, Euphorbia agraria, Euphorbia virgata, Falcaria vulgaris, Linaria biebersteinii, Medicago falcata, Poa angustifolia, Potentilla argentea, Senecio erucifolius, Verbascum lychnitis, Viola kitaibeliana), but only 8 euapophytes represented the above mentioned frequency class (Berteroa incana, Chenopodium album, Cirsium arvense, Consolida paniculata, Convolvulus arvensis, Elytrigia repens, Galium aparine, Melandrium album, Melilotus officinalis). In addition, hemiapophytes were the second most numerous group (95). They were the most abundant group (abundancy 4156, Table 2) of native synanthropic species, as well. Native synanthropes (146 species) were less numerous than non-synanthropes (180). This difference was smaller when the total abundance of species was taken into account (5866 and 5362, respectively, Table 2).

The total number of species of alien origin (anthropophytes) amounted to 113 . They comprised $25,8 \%$ of the flora of kurgans in the Pontic herb(-rich) grass steppe zone and represented 29 families, mainly Asteraceae (17 species), Brassicaceae (17 species), Fabaceae (10), Poaceae (9), Rosaceae (8) and Chenopodiaceae (7). Archaeophytes dominated among alien species $(13,9 \%$ of the total flora of kurgans; the abundance of 61 species of archaeophytes was estimated at 2059). The most frequently occurring archaeophytes (frequency class V) were: Ballota nigra, Buglossoides arvense, Carduus acanthoides, Hyoscyamus niger, Lactuca serriola, Lamium amplexicaule, Lathyrus tuberosus, Onopordum acanthium, Sisymbrium loeselii. Kenophytes were less numerous (42 species; 9,6\%) and their total abundance was estimated to be 711 . No species of kenophytes represented frequency class V; the following species were, however, included in frequency class IV: Ambrosia artemsiifolia, Cardaria draba, Conyza canadensis, Iva xanthiifolia, Rapistrum perenne, Reseda lutea, Rumex patientia, Thlaspi perfoliatum, Tragopogon major. Ergasiophygophytes are a group of species which escaped from the cultivated fields surrounding the kurgans and became temporarily established on the barrows. They, however, made up only $2,3 \%$ of the total flora of kurgans and were practically absent in the other steppe zones.

The synanthropization level of the flora of kurgans estimated from the proportion of native and alien synanthropic species on the kurgans was more or less the same in the three steppe zones studied (in the desert steppe zone: $59,1 \%$, in the grass steppe zone: $60,5 \%$, and in the Pontic herb(-rich) grass steppe zone: 58,6\%). It is interesting to note that the percentage of the particular groups of synanthropic species was comparable in the three steppe zones. However, some differences appeared when the geographical origin of the species was analyzed. Asian species dominated (29\%) in the flora of the Pontic herb(-rich) grass steppe zone. Mediterranean-Eurasian and Mediterranean-European species comprised 25\% and 24\% 
of the flora, respectively. It should be noted that the number of anthropophytes of various origin changed in the south-north direction: in the desert steppe zone, the contribution of Mediterranean-(Eur)asian species was much higher than that of Asian species. In the case of the grass steppe, the differences in the proportion of the above two species groups were less pronounced, whereas in the Pontic herb(-rich) grass steppe zone Asian species were predominant.

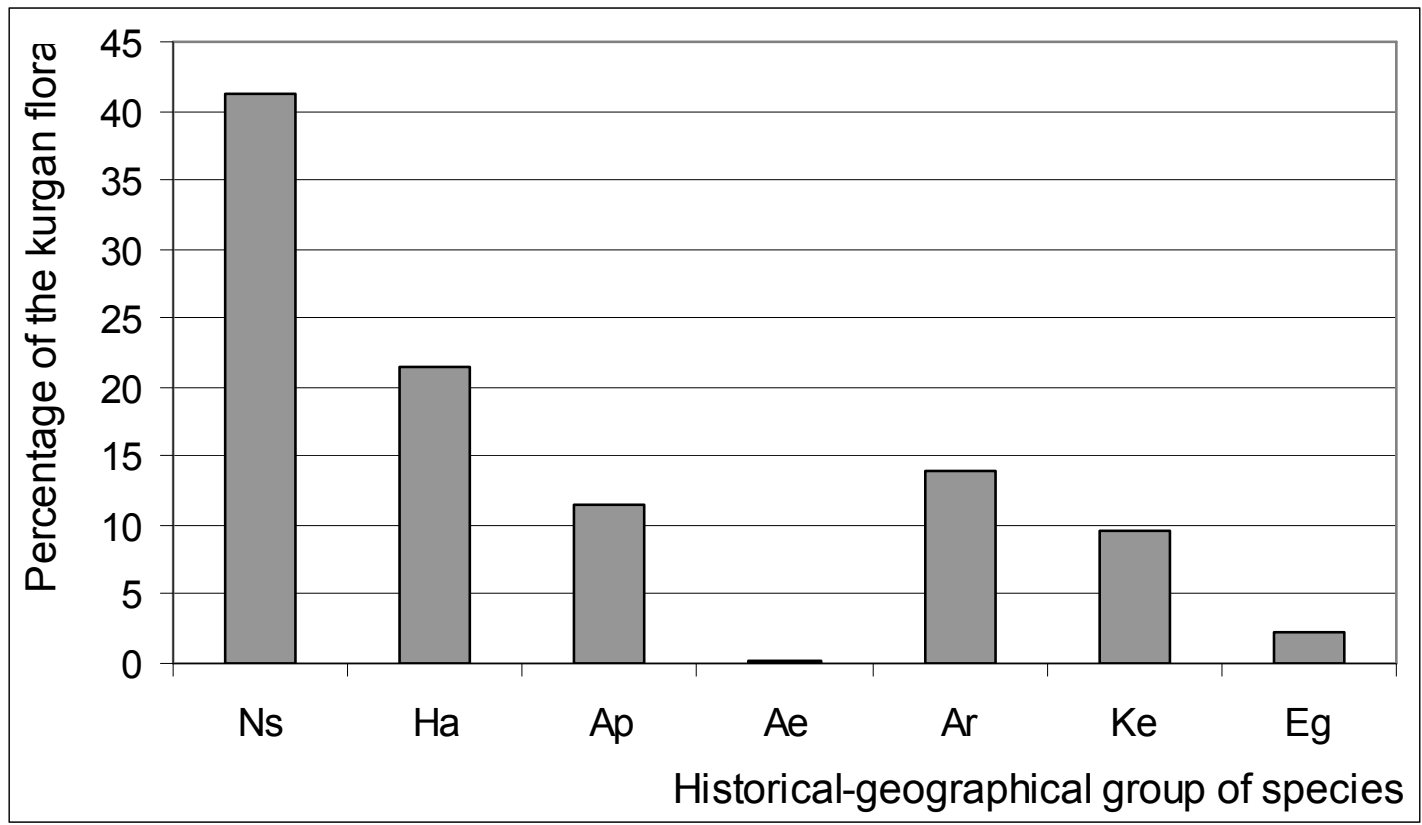

Fig. 6. Historical-geographical-classification of the flora of kurgans in the Pontic herb(-rich) grass steppe zone (for abbreviations see Appendix 1 and Table 2).

Рис. 6. Географічно-історична класифікація флори курганів зони понтичного різнотравно- та багаторізнотравно-злакового степу (прийняті скорочення дивись в Додатку 1 та Таблиці 2).

Table 2

The number and abundance of species in groups of historical-geographical classification of the flora of kurgans in the Pontic herb(-rich) grass steppe zone

Таблиця 2

Кількість і рясність видів в групах географічно-історичної класифікації флори курганів зони понтичного різнотравно- та багаторізнотравно-злакового степу

\begin{tabular}{|c|c|c|c|c|}
\hline \multirow[t]{2}{*}{$\begin{array}{l}\text { Historical-geographical- group in } \\
\text { the kurgan flora }\end{array}$} & \multicolumn{2}{|c|}{$\begin{array}{l}\text { Species in historical- } \\
\text { geographical group }\end{array}$} & \multicolumn{2}{|c|}{$\begin{array}{l}\text { Total abundance of species in } \\
\text { historical-geographical group }\end{array}$} \\
\hline & Number & $\%$ & Number & $\%$ \\
\hline Indigenous species: & 326 & 74,3 & 11228 & 80,1 \\
\hline Non-synanthropes (Ns) & 180 & 41,0 & 5362 & 38,2 \\
\hline Apophytes: & 146 & 33,3 & 5866 & 41,8 \\
\hline - Hemiapophytes (Ha) & 95 & 21,6 & 4156 & 29,6 \\
\hline - Euapophytes (Ap) & 50 & 11,4 & 1704 & 12,2 \\
\hline - Oekiophytes (Ae) & 1 & 0,2 & 6 & 0,04 \\
\hline Anthropophytes: & 113 & 25,7 & 2795 & 19,9 \\
\hline Archaeophytes (Ar) & 61 & 13,9 & 2059 & 14,7 \\
\hline Kenophytes (Ke) & 42 & 9,6 & 711 & 5,0 \\
\hline Ergasiophygophytes (Eg) & 10 & 2,3 & 25 & 0,2 \\
\hline Total flora & 439 & 100 & 14023 & 100 \\
\hline
\end{tabular}




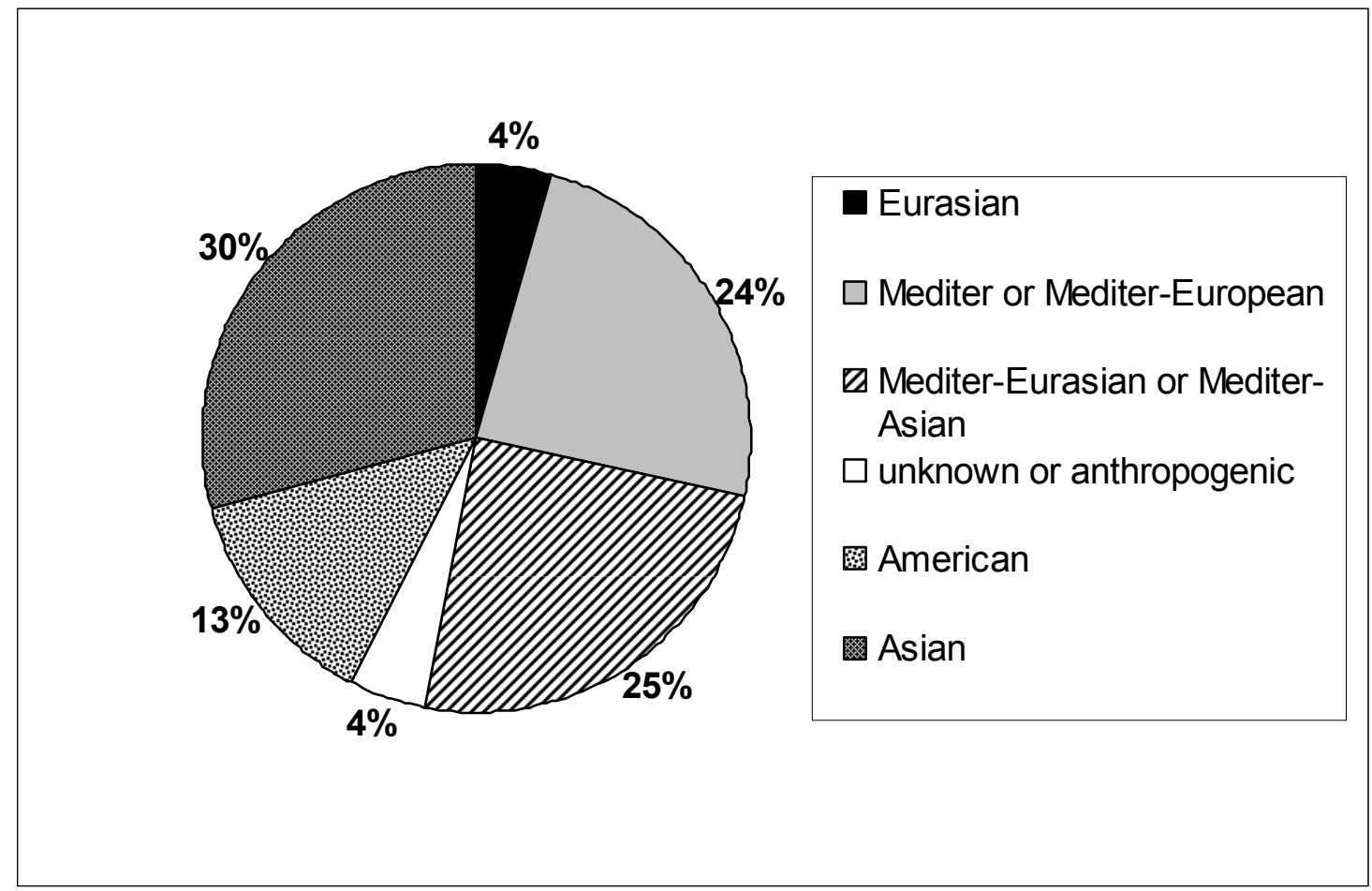

Fig. 7. Origin of the alien flora of kurgans in the Pontic herb(-rich) grass steppe zone.

Рис. 7. Походження адвентивного елементу флори курганів зони понтичного різнотравно- та багаторізнотравно-злакового степу.

Floristic value of the kurgans and conservation of the plant cover

Problems related to the conservation of the flora of kurgans were more widely discussed in our earlier paper [SUDNIK-WÓJCIKOWSKA, MOYSIYENKO, 2006] devoted to the flora of the grass steppe zone. Therefore the present work gives only a short description of the sozophytes (species of special concern) that occurred on the kurgans in the Pontic herb(-rich) grass steppe zone.

Among the species listed (Appendix 1), the majority - over $71 \%$ - were native plants, of which at least 17 should be considered as particularly interesting: Astragalus dasyanthus, Elytrigia stipifolia, Linaria biebersteinii listed in 1997 IUCN - Red List of Threatened Plants" [Мосякін, 1999], Galium volhynicum, Phlomis hybrida and also Astragalus dasyanthus, Elytrigia stipifolia from the "Europeaen Red List" [Червона книга..., 1996], Crocus reticulatus, Stipa capillata, Stipa lessingiana and also Astragalus dasyanthus, Elytrigia stipifolia - from the "Red Data Book of Ukraine" [ЧЕРВОНА КНИГА..., 1996], Adonis vernalis, Amygdalus nana, Anemone sylvestris, Iris halophila, Limonium platyphyllum - from the "Red List of Mykolaiv Region [РЕГІОНАЛЬНИЙ «ЧЕРВОНИЙ» СПИСОК МИКОЛАїВСьКОї ОБЛАСТІ, 1990]" and Goniolimon tataricum, Hesperis tristis, Ornithogalum kochii - from the "Red List of Kirovograd Region" [ЗАПОВДНІ КУТОчКИ КІРОВОГРАДСЬКОї ЗЕМЛІ, 1999].

Although in the zone studied the flora of kurgans was richer in species than that in the grass steppe zone, the number of sozophytes on the barrows was slightly lower (17 and 18 species, respectively) in the Pontic herb(-rich) grass steppe zone. The flora of kurgans in the latter zone was more similar to that of the barrows in the desert steppe zone with respect to the percentage of sozophytes $(3,9 \%$ and $3,3 \%$, respectively). In the case of the grass steppe zone sozophytes comprised $5,1 \%$ of the total flora of kurgans. A certain tendency can, therefore, be observed [МОЙСИЕНКО, СУДНИК-ВОЙЦИКОВСКАЯ 2008]. Among large number of natural communities and those resembling natural ones occurring on the kurgans the steppe communities have been preserved to great extent. The high percentage of sozophytes in the 
flora of kurgans was consistent with the high percentage of steppe species. Their proportion in the flora of kurgans was as follows (from south to north): in the desert steppe zone; $41 \%$ steppe species, 3,3\% sozophytes, in the grass steppe zone: $65,7 \% / 5,1 \%$, and in the Pontic herb(-rich) grass steppe zone: $49,9 \% / 3,9 \%$. The steppe species as well as sozophytes were most numerous in the grass steppe zone, which is located between the other two steppe zones, and practically does not border directly any other zones than the steppe zones. The other two zones have, to some extent, a transitional character. In the south the desert steppe zone merges into the desert (there is no true desert in Ukraine yet; there are, however, intrazonal, halophilous communities characteristic of the seaside zone). Thus the flora is richer in halophyte species, and the role of steppe species decreases. In the north, the Pontic herb(-rich) grass steppe zone gives way to the forest steppe zone. The number of steppe species declines in the above zone as well, whereas the number of forest and meadow species increases. The number of sozophytes recorded on particular kurgans varied from 2 to 9 species (4,5 on average). The most valuable kurgans were R2 and R14, which supported the highest number of sozophytes ( 9 species on each of the kurgans) of all the kurgans investigated in the steppe zone. It should be noted, however, that not only do the kurgans rich in sozophytes need to be protected, but also all the barrows containing fragments of steppe communities that have survived up to the present.

\section{Acknowledgements}

The study was supported by the Ministry of Science and Higher Education in Poland: grant 2 P04G 04627 (2004-2007) and grant NN 304081835 (2008-2011).

\section{References}

БІлик Г.І. Принципи класифікації та класифікаційна схема степів України // Рослинність УРСР. Степи кам'янисті відслонення, піски. - Київ: Наукова думка, 1973. - С. 29-33.

ЗАПОВІДнг КУтОчки КІРОВОГРАДСьКОї ЗЕмЛІ / Андрієнко Т.Л., Терещенко П.С., Клєстов М.Л. та ін. (під заг. ред. д.б.н. Т.Л. Андрієнко). - Київ: Арктур-А, 1999. - 240 с.

ЛАВРЕНКО Е.Н., КАРАМЫШЕВА З.В., НИКУЛИНА Р.И. Степи Евразии. - Ленинград: Из-во «Наука», 1991. $146 \mathrm{c}$.

МАРИнич А.М., ПАщЕНКО В.М., ШищЕНКО П.Г. Природа Украинской ССР. Ландшафты и физикогеографическое районирование. - Київ: Наук. думка, 1985. - 222 с.

МиРКИН Б.М., НАУмОВА Л.Г. Наука о растительности. - Уфа: Гилем, 1998. - 412 с.

Мосякін С.Л. Рослини України у Світовому Червоному списку // Укр. ботан. журн. - 1999. - Т. 56, № 1. C. $79-88$.

Мойсієнко І., Суднгк-ВойциковсьКА Б. Адвентивні рослини на курганах в пустельних степах півдня України // Синантропізація рослинного покриву України (м. Переяслав-Хмельницький, 27-28 квітня 2006). - Тези наукових доповідей. - Київ, Переяслав-Хмельницький, 2006а. - С. 142-144.

МойсиЕнко И., Судник-ВойцыковскА Б. Особенности флоры курганов пустынно-степной зоны Украины // Степи Северной Евразии. Материалы IV Международного симпозиума. Под научной редакцией члена корреспондента РАН А.А. Чибилева. - Оренбург: ИПК «Газпромпечать» ООО «Оренбурггазпромсервис», 2006 б. - С. 481-483.

Мойсієнко І.І., Суднік-ВойциковСьКА Б. Созофіти у флорі курганів - рефугіумів степової флори на півдні України // Заповідна справа в Україні. - Т. 14, Вип. 1. - 2008. - С.16-24.

ПРИРОДА УКРАИНСКОЙ ССР. КЛИМАТ / Бабиченко В.Н., Барабаш М.Б., ЛоГвинов К.Т. и др. (Отв. ред. Логинов К.Т., Щербань М.Г.). - К.: Наукова думка, 1984. - 232 с.

ПриРоДА УКРАИНСКОЙ ССР. Почвы / Вернандер Н.Б., Гоголев И.Н., Ковалишин Д.И. и др. (Отв. ред. Н.Б.Вернандер, Д.А.Тютюнник). - К.: Наукова думка, 1986. - 214 с.

РЕГІОНАЛЬНИЙ «ЧЕРВОНИЙ» СПИСОК МИКОЛАЇВСьКОї ОБЛАСТІ // Рішення III сесії 16 скликання Миколаївської обласної ради депутатів трудящих від 16 жовтня 1990 р. - Миколаїв, 1990. - 15 с.

СоломАХА В.А. Синтаксономія рослинності України // Укр. фітоцен. зб. - Фітосоціоцентр. - Київ, 1996. - Сер. А, вип. 4 (5). -120 c.

ЧЕРвонА кНиГА України / Ю.Р.Шеляг-Сосонко (відп. ред.) та ін. - Київ: Вид-во Укр. енцикл., 1996. $608 \mathrm{c}$.

MAP OF THE NATURAL VEGETATION OF EUROPE. Scale 1: 2500000 [Karte der natürlichen Vegetation Europas, 1:2 500 000] / Bohn U., Gollub G., Hettwer C. \& al. - Bonn: Bundesamt für Natürschutz, 2000. Maps: 9 sheets. - Legend: 153 p. 
MATUSZKIEWICZ W. Przewodnik do oznaczania zbiorowisk roślinnych Polski. - Warszawa: Wydawnictwo Naukowe PWN, 2001. - 537 p.

Mosyakin S. L., FedoronchuK M. M. Vascular plants of Ukraine. A nomenclatural checklist. - Kiev: M. G. Kholodny Institute of Botany, National Academy of Sciences of Ukraine, 1999. - $346 \mathrm{p}$.

Moysiyenko I.I., SudniK-Wójcikowska B. The Flora of Kurgans in the Desert Steppe Zone of Southern Ukraine. Chornomors'k. bot. z. - 2006. - Vol. 2, N 1. - P. 5-35.

SudNiK-WóJCIKOWska B., MoysiYenko I.I. The Flora of Kurgans in the West Pontic Grass Steppe Zone of Southern Ukraine. Chornomors'k. bot. z. - 2006. - Vol. 2, N 2. - P. 14-44.

SUDNIK-WóJCIKOWSKA B., MoYSIYENKO I.I. The floristic differentiation of microhabitats within kurgans in the desert steppe zone of southern Ukraine // Acta Soc. Bot. Pol. - 2008. - Vol. 77, N 2. - P. 139-147.

Рекомендує до друку

Отримано 21.08.2009 p.

$$
\text { A.В. Сна }
$$

Aдpeca автора:

I.I. Мойсієнко

Кафедра ботаніки,

Херсонського державного університету,

Вул. 40 років Жовтня, 27, 73000 Херсон,

Україна

e-mail: Vanvan@ksu.ks.ua

\section{Adpeca aвmopa:}

Barbara Sudnik-Wójcikowska,

Zakład Ekologii Roślin i Ochrony Środowiska,

Uniwersytet Warszawski,

Al. Ujazdowskie 4,

00-478 Warsaw, Poland;

e-mail: barbara.sudnik@uw.edu.pl
Author's address:

Ivan Moysiyenko,

Department of Botany,

Kherson State University,

Str. 40 let Oktriabrya 27, 73000 Kherson,

Ukraine

e-mail:Vanvan@ksu.ks.ua

Author's address:

Barbara Sudnik-Wójcikowska,

Department of Plant Ecology and Environmental Conservation,

University of Warsaw, Al. Ujazdowskie 4,

00-478 Warsaw, Poland;

e-mail: barbara.sudnik@uw.edu.pl 


\section{APPENDIX 1. Flora of the kurgans in desert steppe zone and their microhabitats}

Abbreviations applied in Table:

Microhabitats:

$\mathrm{T}$ - the top of the barrow;

Ss - the southern slopes;

$\mathrm{Sn}$ - the northern slopes;

Bs - the southern foot;

$\mathrm{Bn}-$ the northern foot.

Data regarding the occurrence of species in particular microhabitats are presented in the following order:

\begin{tabular}{|l|l|}
\hline \multicolumn{2}{|c|}{ T } \\
Ss & Sn \\
Bs & Bn \\
\hline
\end{tabular}

Life forms:

$\mathrm{t}$ - therophytes;

th - short-living perennials (2,3,4 years old);

g-geophytes;

w - hydrophytes;

h - hemicryptophytes;

hg - geophytes-hemicryptophytes - perennials, some of whose perennating buds (shoot system) remain on the soil surface and underground;

hc - hemicryptophytes-chamaephytes - perennials whose perennating buds remain on or above (within 0.25 m) the soil surface;

c - chamaephytes;

$\mathrm{mf}$ - megaphanerophytes;

$\mathrm{nf}$ - nanophanerophytes;

\section{Syntaxa:}

Agro int-rep - Agropyretea intermedio-repentis (Oberd. et al. 1967) Müller et Görs 1969)

Alne glut-Alnetea glutinosae Br-Bl. et R. Tx. 1943

Alth offi-Althaetalia officinalis V. Golub et Mirkin in V. Golub 1995 \{Molinio-Arrhenatheretea T. Tx 1937 \}

Ammoph-Ammophiletea Br.-Bl. et R.Tx. 1943

Artemi-Artemisietea vulgaris Lohm., Prsg et R. Tx. in R. Tx. 1950

Aste trip - Asteretea trifolium Westh. et Beeft. ap. Beeft. 1962

Bident-Bidentetea tripartiti R.Tx., Lohm. et Prsg. 1950

Caki mari-Cakiletea maritimae R. Tx. et Prsg. 1950

Crit-Limo - Crithmo-Limonietea Br.-Bl. 1947

Crit-Stat-Crithmo-Staticetea Br.-Bl. 1947

Fest vagi-Festucetea vaginatae Soy 1968 em. Vicherek 1972 or Festucetalia vaginatae Soy\{FestucoBrometea Br.-Bl. et R. Tx. 1943 \}

Fest-Brom - Festuco-Brometea Br.-Bl. et R. Tx. 1943

Fest-Pucc-Festuco-Puccinellietea Soy (incl. Festuco-Limonietea Karpov et Mirkin 1985)

Gali veri-Galietalia veri Mirkin et Naumova 1986 \{Molinio-Arrhenatheretea T. Tx. 1937\}

Gali-Urti-Galio-Urticetea Passarge 1967 or Galio-Urticenea (Passarge 1967) \{Artemisietea Lohm., Prsg. et R. Tx. in R. Tx. 1950\}

Glecho-Glechometalia hederaceae R. Tx. in R. Tx.

Moli-Arrh-Molinio-Arrhenatheretea R. Tx. 1937

Phragmi-Phragmitetalia Koch 1926

Plan majo - Plantaginetea majoris T. Tx. et Prsg. 1950 or Plantaginetalia majoris R. Tx. (1943) 1950 \{Molinio-Arrhenatheretea $\mathrm{R}$. Tx. 1937\}

Poly-Arte - Polygono-Artemisietea austriacae Mirkin, Sakhapov et Solomeshch in Mirkin et al. 1986

Quer rob-pe-Quercetea robori-petraeae Br.-Bl. et R. Tx. 1943

Quer pub-pe-Quercetalia pubescenti-petraeae Klika 1933 corr. Moravec in Beg. et Theurill 1984

Quer-Fage-Querco-Fagetea Br.-Bl. et Vlieg. 1937

Rham-Prun - Rhamno-Prunetea Rivas, Goday et Garb. 1961\}

Robin - Robinietea Jurko ex Hadac et Sofron 1980

Sali purp - Salicetea purpureae Moor 1958

Sedo-Scle-Sedo-Scleranthetea Br.-Bl. 1955

Stel medi-Stellarietea mediae T. Tx. , Lohm., et Prsg. 1950 (incl. Chenopodietea Br.-Bl. 1952 em. Lohm., J. et R. Tx. 1961 ex Matuszk.1962 \& Secalietea Br.-Bl. 1951) 
Trif-Gera - Trifolio-Geranietea sanguinnei Th. Müller 1962

Urti-Samb - Urtico-Sambucetea Doing1962 em. Pass.1968

Vacc-Pice - Vaccinio-Piceetea Br-Bl. 1939

[ ] - indicates that the introduced species were established in the particular plant community types (see Table);

\{\} - indicates that the syntaxon belongs to a given class (see above list of syntaxa abbreviations).

Historical-geographical classification of species:

Native species:

Ns - native species, not established in anthropogenic habitats;

$\mathrm{Ap}-$ proper apophytes $=$ euapophytes, natives established in anthropogenic habitats;

Ha- hemiapophytes, natives established only in semi-natural habitats;

$\mathrm{Ae}$ - oekiophytes, natives grown (e.g. in plantations or in windbreaks) and recorded in anthropogenic habitats.

Aliens:

$\mathrm{Ar}-$ archaeophytes, aliens that immigrated before the year 1500;

$\mathrm{Ke}$ - kenophytes, aliens introduced after the year 1500 ;

Eg - ergasiophygophytes, cultivated plants not established in the new territory, appearing only temporarily.

Origin of alien species - groups and abbreviations:

1 Mediterr (= Mediterranean), sub-Mediterr (= sub-Mediterranean);

2 European, Atlantic, sub-Atlantic;

3 Eurasian, Eurosiberian, boreal-Eurasian, continent. (= continental), subcontinent (= subcontinental);

4 W-Asian (=Western-Asian), Middle-Asian, C-Asian (= Central Asian), Irano-Turanian, Indian, Malay;

5 African,

6 North American, Central American, South American.

Status of the protected species:

* - World Red List

** - European Red List

*** - Red Data Book of Ukraine

**** - Red Lists of Mykolaiv Region and Kirovograd Region

At the bottom of the Table the flora of each kurgan is described taking into account:

1 the number of species;

2 the number of species in all of its microhabitats. 


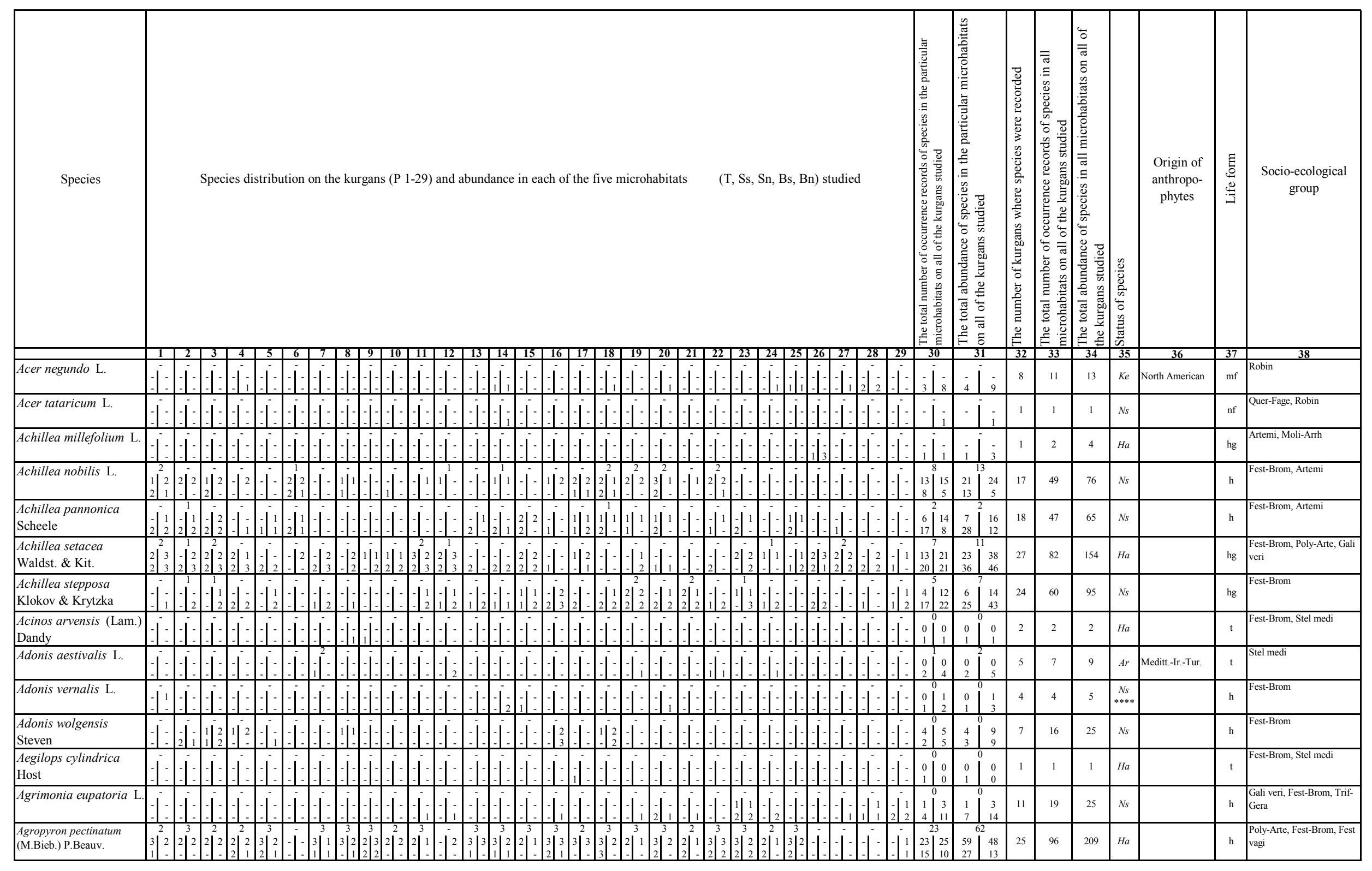




\begin{tabular}{|c|c|c|c|c|c|c|c|c|c|c|c|c|c|c|c|c|c|c|c|c|c|c|c|c|c|c|}
\hline Igrostemma githago $\mathrm{L}$ & 11 & & & & & & & & $2913+14$ & & $\begin{array}{ll}-1 \\
-i & -18 \\
\end{array}$ & 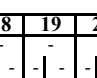 & & & 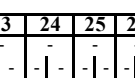 & & & $\frac{30}{010}$ & & \begin{tabular}{|c|c|}
32 \\
0 & 1 \\
\end{tabular} & $\frac{33}{2}$ & & & & & SStel medif 38 \\
\hline & $1:$ & & & - & & & : : & - & & & & & & & & & $:$ & {$\left[\begin{array}{lll}0 & 0 \\
1 & 1 & 1 \\
1\end{array}\right.$} & & \begin{tabular}{l|l|l}
0 & 1 \\
2 & & \\
2
\end{tabular} & ${ }^{2}$ & & ${ }^{A r}$ & & & \\
\hline fjugg chia Schreb. & : & & & 1 & & & $\vdots$ & & & & & -1 & & & $:=$ & & 1 & $\begin{array}{l}81 \\
8\end{array}$ & \begin{tabular}{c|c}
3 & 8 \\
9 & 8 \\
9
\end{tabular} & \begin{tabular}{|l|l|}
8 & 13 \\
3 & 13 \\
\end{tabular} & 25 & 26 & Ha & & he. & st:Brom, S \\
\hline Ajuga genevensis L. & 11 & & & & & & . & & & & & & & & -1 & & & & & \begin{tabular}{l|l}
1 & 13 \\
\end{tabular} & 20 & 24 & $N_{s s}$ & & $\mathrm{~h}$ & \\
\hline Alcea rosea $\mathrm{L}$. & : & & & 1 & & & : & . & & & & : & & & $::$ & & 1 & 00 & \begin{tabular}{l|l|l|}
0 & 0 & 0 \\
0 & 0 & 0 \\
\end{tabular} & $\begin{array}{ll}0 & 1 \\
0 & \end{array}$ & 1 & 1 & $E_{g}$ & funknown origin & $\mathrm{h}$ & Aremi \\
\hline \begin{tabular}{|l} 
Allium flavescens \\
Besser
\end{tabular} & 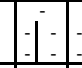 & i: il & & 1 & & & $1^{2}$ & 1 & & & & $=2_{2}^{2}=1$ & & & $:$ & & . & 73 & \begin{tabular}{c|c|c}
5 & 9 \\
& 9 & 9 \\
1
\end{tabular} & $\begin{array}{lll}9 & 8 \\
\end{array}$ & 20 & 28 & $N_{s}$ & & $\mathrm{~g}$ & Fat-Brom \\
\hline \begin{tabular}{|l|l|l} 
Allium paczoskianum \\
Tuzs.
\end{tabular} & 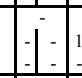 & : & - & : & & & 1 & 1 & & & 1 & 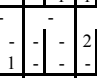 & & & : & & : & $\begin{array}{lll}6 & 0 \\
6 & 4 \\
0 & 4 \\
1\end{array}$ & 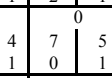 & ${ }^{10}$ & "1 & 13 & $N_{s}$ & & $\mathrm{~g}$ & Fest-Brom \\
\hline Allium paniculatum L. & $1:$ & : & & : & & & is & $:$ & & & & & & & $1: 1:$ & & it & $\begin{array}{l}2 \\
0\end{array}$ & \begin{tabular}{l|l|l}
7 & 3 \\
2 & 3 & 12 \\
0
\end{tabular} & 8 & 16 & 23 & $N_{s s}$ & & $\mathrm{~g}$ & \\
\hline $\begin{array}{l}\text { Alliuiur rotundium L. } \\
\text { aggr. }\end{array}$ & i & $i_{1}^{1}$ & 1 & 1 & 1 & & : & $: 1$ & & & & : & & & i & 1 & : & $\begin{array}{ll}3 \\
3 \\
6\end{array}$ & \begin{tabular}{l|l|l}
3 & 3 \\
6 & 3 & 6 \\
9 & 10 \\
\end{tabular} & $\begin{array}{ll}6 \\
6 \\
0\end{array}$ & 23 & ${ }^{31}$ & ${ }_{N s}$ & & $\mathrm{~g}$ & Fest-Brom, Gali veri \\
\hline Mlopecurus pratensis & $i_{2}$ & il: & & : 1 & & & i: & $: 1$ & $\therefore$ & & & $1:$ & & & $: 1$ & $=1$ & E: & $\begin{array}{lll}0 \\
0 \\
0\end{array}$ & \begin{tabular}{c|c|c}
0 \\
0 \\
3
\end{tabular} & \begin{tabular}{l|l}
0 \\
\\
5
\end{tabular} & 4 & ${ }^{6}$ & $N_{s s}$ & & $\mathrm{~g}$ & \\
\hline 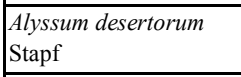 & $1:$ & & 1 & $: 1$ & & & : & - & & & $\begin{array}{lll}2 & \\
2 & 2\end{array}$ & 2 & & & in & 1 & : & $\begin{array}{ll}5 \\
5 \\
3 \\
3\end{array}$ & 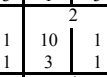 & $\begin{array}{ll}9 \\
1\end{array}$ & 12 & 17 & $H a$ & & & 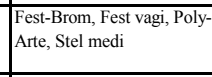 \\
\hline $\mid \begin{array}{l}\text { Alyssum hirsutum } \\
\text { M..iieb. }\end{array}$ & $1:$ & $2=2$ & : & : & & & : & $\therefore$ & & & & $: 1$ & & & & & $\therefore$ & $\left.\begin{array}{l}2 \\
2 \\
0\end{array}\right]_{0}^{0}$ & \begin{tabular}{l|l|l|l} 
& 4 & 0 \\
0 & 0 & 0 \\
0 & 0 & 0
\end{tabular} & \begin{tabular}{l|l}
0 & 2 \\
0 &
\end{tabular} & ${ }^{4}$ & ${ }^{8}$ & Ha & & & 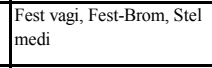 \\
\hline Amaranthus albus $\mathrm{L}$. & 1 & i: & & 1 & . & & i & :1: & & & & & & & & & $\therefore$ & $\begin{array}{l}0 \\
1 \\
1\end{array}$ & \begin{tabular}{ll|l}
0 & 0 \\
0 & 0 & 0 \\
1 & 1 & 1
\end{tabular} & \begin{tabular}{l|l}
0 & 1 \\
&
\end{tabular} & 2 & ${ }^{2}$ & ке & $\begin{array}{ll}-2 & \text { North American } \\
\end{array}$ & & Stel matit \\
\hline $\begin{array}{l}\text { timaranchus } \\
\text { s. Watson }\end{array}$ & $1:$ & i: & & -1 & & & : & : & & & & $1:$ & & & -1 & -1 & : & $\because 0$ & $\begin{array}{llll}0 & 0 \\
0 & 0 & 0 \\
0 & 0 & 1 \\
1\end{array}$ & $\begin{array}{ll}0 \\
1 \\
1\end{array}$ & 1 & 1 & ке & South American & $\mathrm{t}$ & Stell medi \\
\hline $\begin{array}{l}\text { Amaranthus retroflexus } \\
\text { L. }\end{array}$ & $:$ & i i & 1 & : & - & & i & $: 1$ & & & $=1$ & 1 & il! & : & i: & ii & 1 & $\frac{2}{2}$ & $2 \frac{12}{2}+\frac{2}{7}$ & \begin{tabular}{|l|l|}
2 & 13 \\
\end{tabular} & 17 & 18 & Ke & 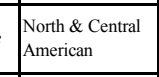 & $\mathrm{t}$ & Stelimedit \\
\hline 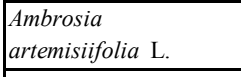 & $1:$ & it: & & -1 & 1 & & itii & $:$ & & & in & $1:$ & 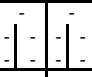 & : & $i=1$ & it & 1 & {$\left[\begin{array}{l}0 \\
7 \\
7\end{array}\right.$} & $\begin{array}{llll}2 & & 1 \\
11 & 0 & 2 \\
7 & 12\end{array}$ & \begin{tabular}{|l|l|}
2 & 17 \\
\end{tabular} & ${ }^{21}$ & 22 & Ke & North American & & Stellmmati \\
\hline 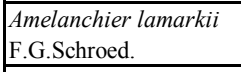 & -1 & & & : & & & : & : & & & & : : & 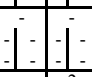 & & & & : & 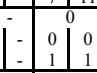 & $\begin{array}{llll}0 & 0 & 0 \\
1 & 0 & 0 \\
1 & 1 & 1\end{array}$ & $\begin{array}{ll} \\
\vdots\end{array}$ & ${ }^{2}$ & ${ }^{2}$ & $E_{8}$ & North American & nf & Robin \\
\hline Amygdalus nana $\mathrm{L}$. & $1:$ & i & & il & & & $\therefore$ & $\therefore$ & & & it & 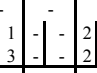 & $22^{2}$ & & : & $: 1$ & : & \begin{tabular}{|l|l|l} 
\\
3 \\
9 & 8 \\
10 & \\
\end{tabular} & 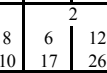 & \begin{tabular}{|l|l|}
12 & 10 \\
2 &
\end{tabular} & 31 & ${ }_{63}$ & 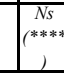 & & nf & Fest-Brom \\
\hline 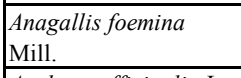 & 1 & : & & -1 & & & 1 & 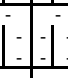 & : & & : & -1 & 1 & & : & & 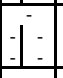 & 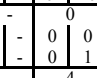 & \begin{tabular}{|l|lll}
0 & 0 & 0 \\
0 & 0 & 0 \\
\end{tabular} & \begin{tabular}{|l|l|} 
& 1 \\
\end{tabular} & 1 & 1 & Ar & Medititer & & Stel menti \\
\hline Anchusa officinalis $\mathrm{L}$. & $2 \begin{array}{ll}2 \\
2 & 1 \\
1 & 1\end{array}$ & $\begin{array}{lll}2 & 2 \\
2 & 1 \\
2 & 1\end{array}$ & & & & & 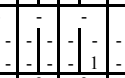 & : & $i_{i}^{1}$ & & $1-1$ & i: & 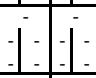 & & & & 1 & {$\left[\begin{array}{l}3 \\
8\end{array}\right]_{6}^{5}$} & $\begin{array}{l}5 \\
6 \\
62 \\
12 \\
6 \\
6 \\
6\end{array}$ & $\begin{array}{c}5 \\
6\end{array}$ & 26 & ${ }^{34}$ & ${ }_{A r}$ & Mediter & & 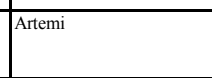 \\
\hline $\operatorname{ata} \mathrm{L}$. & $2 \begin{array}{c}2 \\
2\end{array} \mid 1$ & & & & & & 2 & 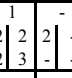 & & & & & & & & & -1 & 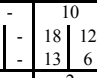 & $\begin{array}{l}39 \\
20 \\
210 \\
10\end{array}$ & $\begin{array}{ll}21 & 21 \\
10 & \end{array}$ & 59 & 113 & ${ }^{N s}$ & & & 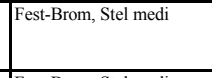 \\
\hline 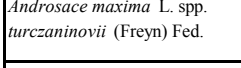 & : & 1 & & t & & & i: & - & & & 3 & 1 & it & & $:$ & & it & $\left.\begin{array}{l}4 \\
2\end{array}\right]_{2}^{2}$ & $\left.\begin{array}{ll}0 \\
0\end{array} \frac{2}{3}\right]_{3}^{3}$ & \begin{tabular}{|l|l|}
3 & 7 \\
3 &
\end{tabular} & 12 & 17 & $\nu_{s}$ & & $\mathrm{t}$ & \\
\hline inemone sylvestris $\mathrm{L}$. & : & & & & & & E: & & & & & & & & & & & $\begin{array}{lll}0 & 0 \\
0 & 0 \\
0 & 1 & \\
1\end{array}$ & $\begin{array}{llll} & 0 \\
0 & 0 & 0 \\
0 & 2 & 2 \\
\end{array}$ & 1 & 1 & 2 & $\cdots$ & & & 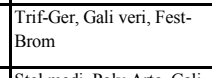 \\
\hline $\begin{array}{l}\text { Anisantha tectorum } \\
\text { L.) Nesski }\end{array}$ & iil: & H. & & & & & 期: : & & & & & & & & & & & & & \begin{tabular}{l|l|l} 
& 4 \\
\end{tabular} & ${ }^{6}$ & 7 & & $\begin{array}{l}\text { Meditier-Irano- } \\
\text { Turunian }\end{array}$ & & 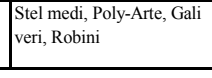 \\
\hline
\end{tabular}




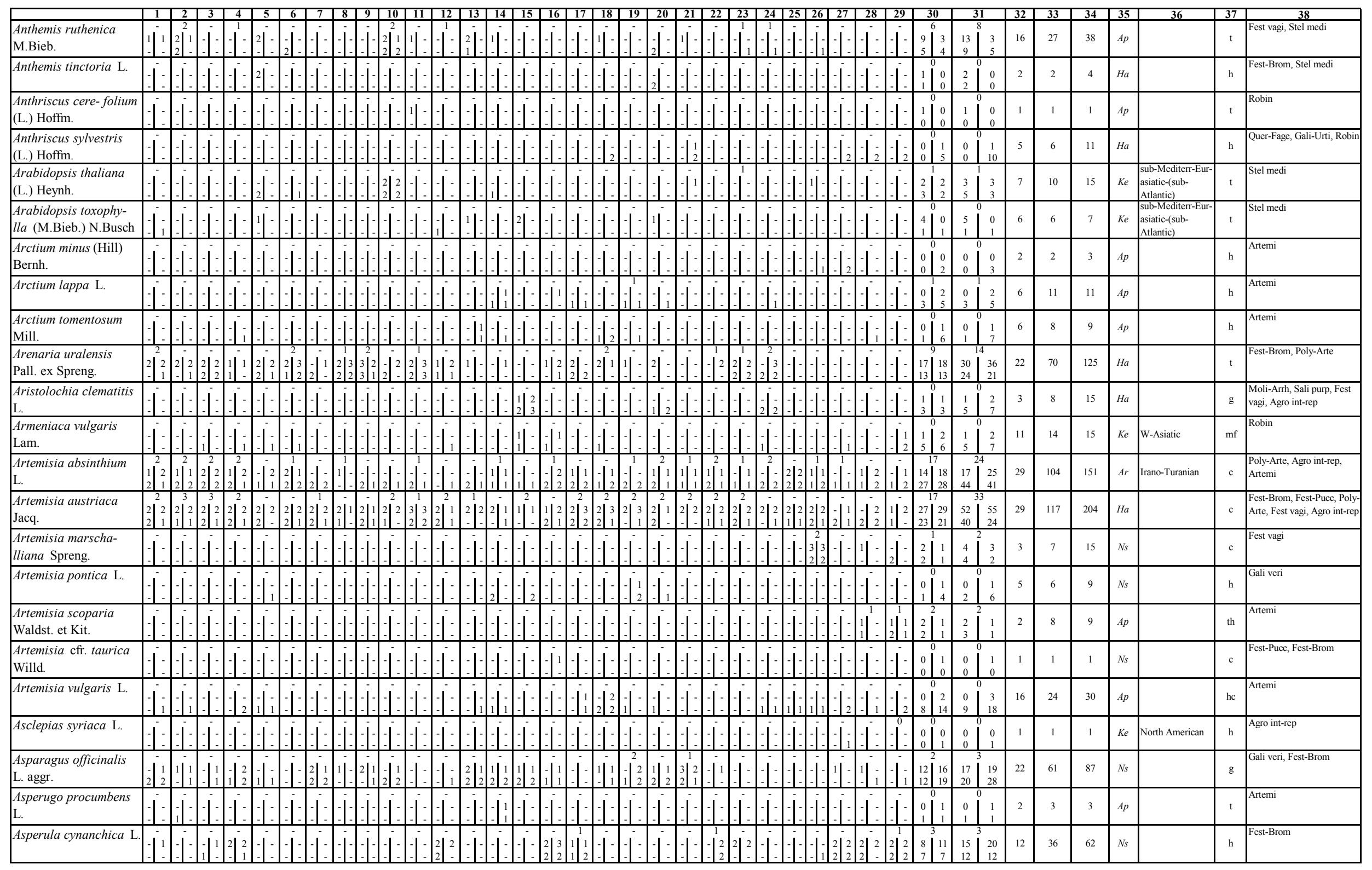




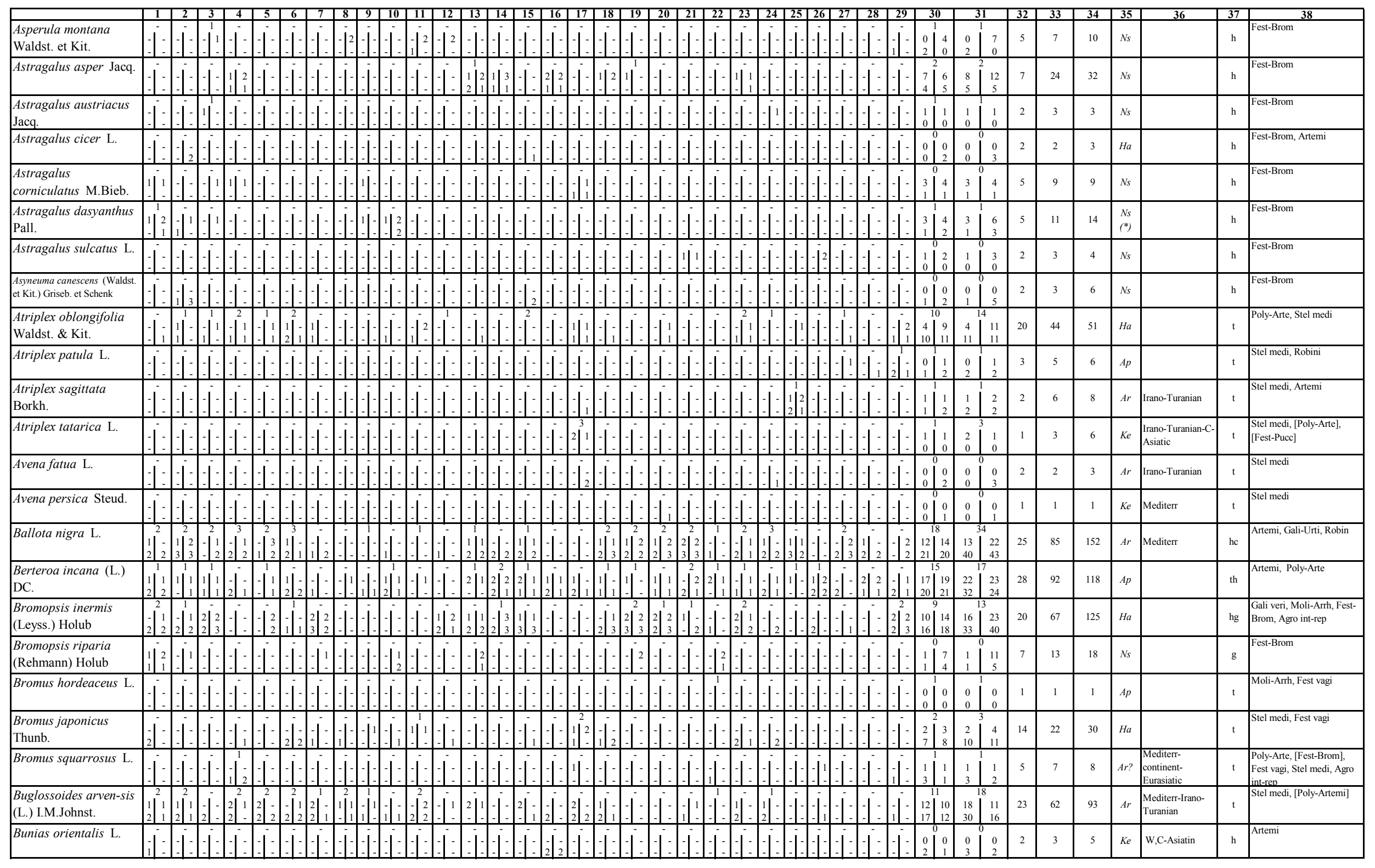




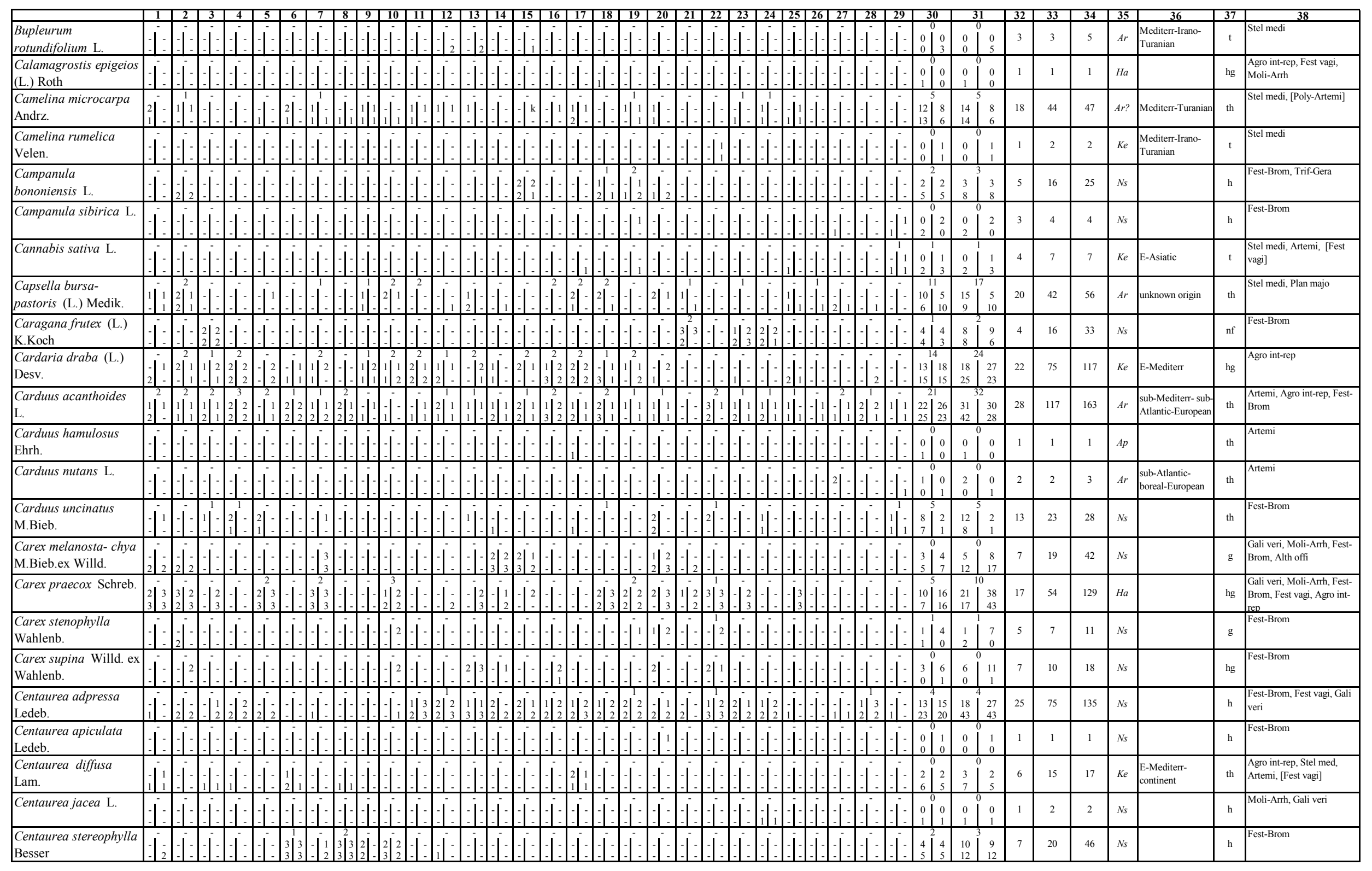




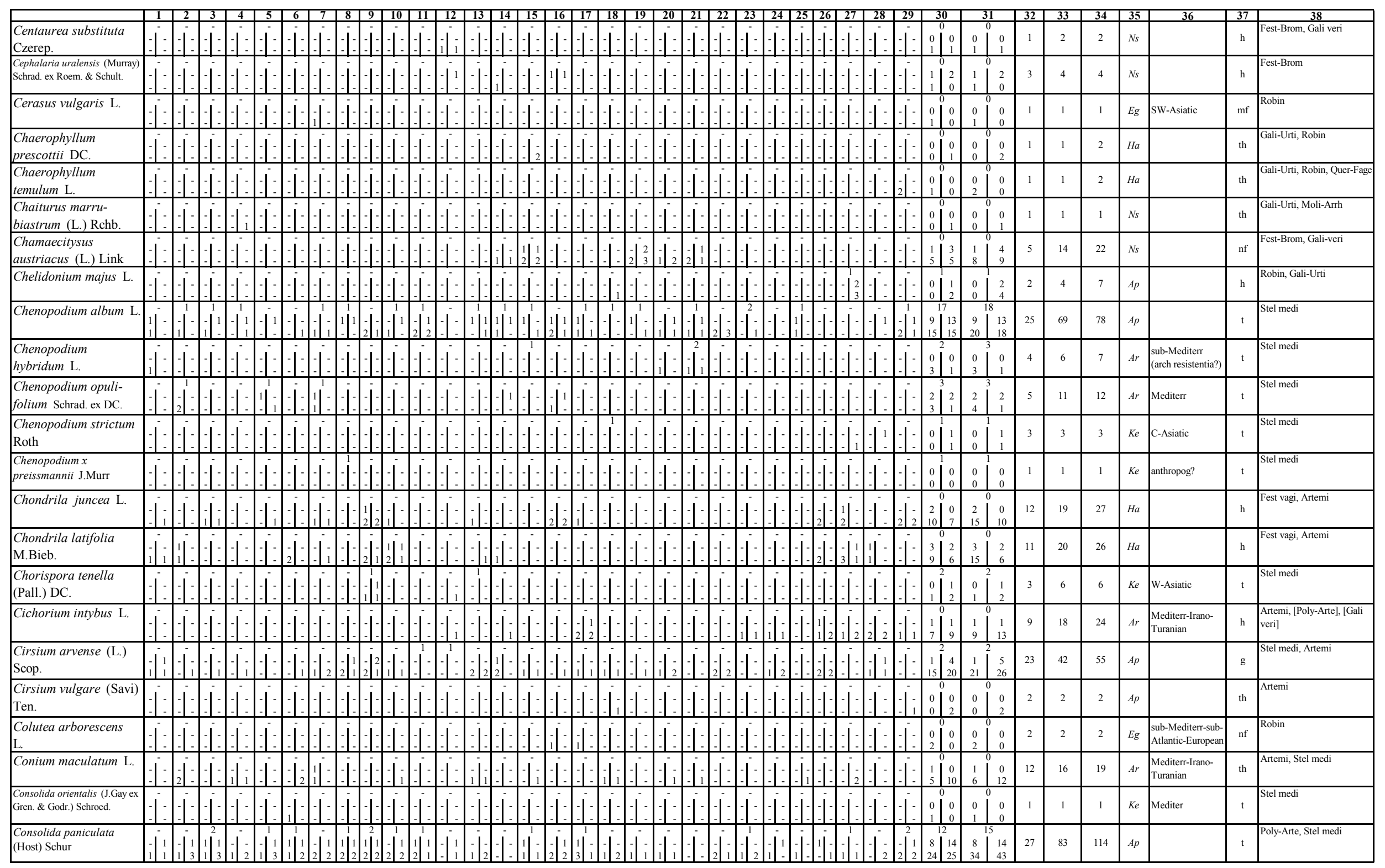




\begin{tabular}{|c|c|c|c|c|c|c|c|c|c|c|c|c|c|c|c|c|c|c|c|c|c|c|c|c|c|c|c|c|c|}
\hline Convolvulus arvensis & & & & & & & & & & & & & & & & & & & & & & & & & & & 36 & & 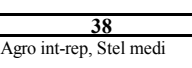 \\
\hline & & & & & & & & & & & & & $\mid \begin{array}{ll}1 \\
2\end{array}$ & & & & & & & $\begin{array}{l}122 \\
2628\end{array}$ & 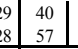 & $\begin{array}{c}50 \\
59 \\
59\end{array}$ & ${ }^{29}$ & 127 & 249 & ${ }^{A p}$ & & hg & \\
\hline $\begin{array}{l}\text { Conzza candensis (L.) } \\
\text { Cronq. }\end{array}$ & & : & t. & & i & & 2] & & : & & $\mathrm{i}$ & $1:$ & il & & & & & & & {$\left[\begin{array}{cc}6 \\
12\end{array}\right]^{5}$} & \begin{tabular}{|c|c|}
6 \\
17 \\
17
\end{tabular} & $\begin{array}{l}5 \\
7 \\
\end{array}$ & 21 & 33 & 40 & ${ }_{\mathrm{Ke}}$ & Norh American & th & \\
\hline Coronilla varia $\mathrm{L}$. & & & & & & & & & & & & 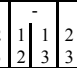 & 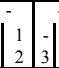 & 2 & & & & : & & 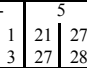 & $\begin{array}{ll}78 \\
78\end{array}$ & $\begin{array}{c}51 \\
64 \\
64\end{array}$ & 28 & 108 & 215 & Ha & & & \\
\hline $\begin{array}{l}\text { Cratacegus monogyna } \\
\text { Jacq. }\end{array}$ & 1 & 1 & | & & & & -1 & & : & & I: & $1:$ & $: 1$ & . & $I^{-}$ & & & & |. & \begin{tabular}{|l|l|l}
0 & 0 \\
2 & 0 & 1 \\
1
\end{tabular} & 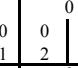 & i & 2 & 3 & 3 & Ns & & nf & hamprerun \\
\hline Crataegus sp. & & : & L & & & & : : & & : & & $1:$ & ii: & 1 & i & : & if & & 1: & $=1$ & $\begin{array}{lll}0 & 0 \\
2 & 1 \\
2 & 1 \\
\end{array}$ & 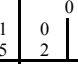 & $\frac{1}{s}$ & 6 & 8 & 8 & $N_{s}$ & & $\mathrm{nf}$ & \\
\hline 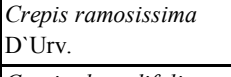 & & i & - & $\therefore$ & L: & & -1 & & $=1$ & & i: & $1:$ & $: 1$ & 1 & 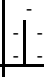 & & & & $|-i| . i$ & $\left.\frac{1}{2}\right|_{0} ^{2}$ & \begin{tabular}{|c|c|}
1 \\
2
\end{tabular} & $0_{0}^{2}$ & 5 & 5 & ${ }^{5}$ & Ha & & $\mathrm{t}$ & \\
\hline \begin{tabular}{|l}
$\begin{array}{l}\text { Crepis rhoeadifolia } \\
\text { M.Bieb. }\end{array}$ \\
\end{tabular} & & & i i & & !' & & & & : & & i: & Vi: & $=1$ & 1 & ]. & & & : : & $: 1$ & $\left.\mid \begin{array}{l}4 \\
6\end{array}\right]^{3}$ & \begin{tabular}{|l|l|}
4 \\
8
\end{tabular} & $\begin{array}{l}3 \\
7 \\
\end{array}$ & 14 & ${ }^{21}$ & ${ }^{23}$ & ${ }^{A p}$ & & ' & Fest vag, A, Atemii \\
\hline \begin{tabular}{|l} 
Crocus reticulatus \\
Steven ex Adams \\
\end{tabular} & & : & 1 & & : & & : & & $=2$ & & 1 & $1:$ & $: 1$ & 1 & - & & & 1:1: & 1 & $\begin{cases}1 \\
0.12 \\
0 \\
0\end{cases}$ & $\begin{array}{ll}20 \\
0 \\
0\end{array}$ & $\begin{array}{l}3 \\
0 \\
0 \\
\end{array}$ & 2 & ${ }^{3}$ & ${ }^{5}$ & $\stackrel{N s}{\cdots}$ & & 8 & Fest-Brom \\
\hline $\begin{array}{l}\text { Cuscuta a } \\
\text { Bab. }\end{array}$ & 1 & : & : & $:$ & : & & 1 & & : & & : & $1:$ & $: 1$ & 1 & : & I & & : & $=1:=1$ & $\begin{array}{lll}1 & 1 \\
1 & 1 \\
0\end{array}$ & \begin{tabular}{l|l}
1 & 2 \\
1 & 1 \\
\end{tabular} & $\begin{array}{c}1 \\
0 \\
0\end{array}$ & 1 & ${ }^{3}$ & ${ }^{4}$ & $N_{s}$ & & t & \\
\hline $\begin{array}{l}\text { Cynoglossum officinale } \\
\end{array}$ & & i & $\frac{1}{2}$ & & i & & & & i: & & ii & i: & 1 & 1 & il & & & H & & 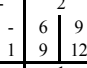 & $\mid$\begin{tabular}{|l}
6 \\
62 \\
12 \\
\end{tabular} & $\stackrel{9}{14}$ & 17 & 38 & ${ }^{43}$ & Ar & $\begin{array}{l}\text { Elarasiacici } \\
\text { continent }\end{array}$ & h & \\
\hline Dactylis glomerata L. & & 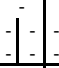 & 1 & & L & & & & & & & : & 1 & 1 & & & & & & $\left(\begin{array}{lll}0 & 1 \\
1 & 3 & 3 \\
\end{array}\right.$ & \begin{tabular}{|l}
0 \\
1 \\
1
\end{tabular} & $\frac{1}{5}$ & 4 & ${ }^{6}$ & 8 & ${ }_{1 p}$ & & hg & Moli-Arnh \\
\hline Datura stramonium L. & & i & 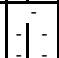 & -1 & i. & & 1 & & i i i & & 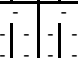 & $\%$ & 1 & 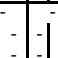 & & 1 & & 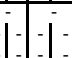 & $\%$ & 00 & 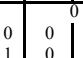 & 0 & 1 & 1 & 1 & ${ }_{A r}$ & SE-Asiatic & i & Stel Inedi \\
\hline Daucus carotata L. & & 1 & i & & 1: & & & & & & & & -1 & 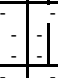 & & & & iil: & & $\left.\begin{array}{|ccc}0 \\
0 \\
5\end{array}\right]^{3}$ & \begin{tabular}{|l|l|}
3 & 0 \\
0 & 0 \\
5 & 2 \\
\end{tabular} & 11 & 12 & 19 & 21 & Ap & & $\mathrm{h}$ & Moli-Arnh, Artemi \\
\hline $\begin{array}{l}\text { (L.) Webb ex Prantl } \\
\end{array}$ & -1 & : & 1 & $\therefore$ & $:$ & & il & & $:=1$ & & $1:$ & 1 & $: 1$ & 1 & $1-1$ & & & $|:|: \mid$ & $1:=1$ & {$\left[\begin{array}{ll}0 \\
6 \\
3\end{array}\right]^{4}$} & \begin{tabular}{l|l|}
4 & 6 \\
2 & 12 \\
\end{tabular} & ${ }_{2}^{4}$ & 12 & ${ }^{23}$ & 28 & ${ }_{A r}$ & trano-Turanian & : & Stel medit \\
\hline $\begin{array}{l}\begin{array}{l}\text { lianthus campestris } \\
\text { M.B.Beb. }\end{array} \\
\end{array}$ & j & & i & & L & & & & : & & i: & i: & : & 1 & & ] & & $a_{1}^{2} 2_{1}^{2}$ & $1: 1$ & $:\left|\frac{3}{2}\right|_{2}^{3}$ & \begin{tabular}{|l|l|}
3 & 6 \\
2 & 1 \\
2
\end{tabular} & ${ }_{2}^{4}$ & ${ }^{3}$ & 11 & 15 & ${ }^{N s}$ & & ${ }^{\mathrm{h}}$ & Fentrion \\
\hline 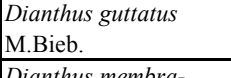 & & ii & 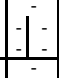 & & : & & $\mid \begin{array}{l}2 \\
2\end{array}$ & & $=\frac{2}{2}$ & & : & $1 !$ & $:=1$ & $\vdots$ & $l_{1}^{2}$ & 1 & & 汭: & $\therefore:=1$ & $1: 56{ }_{6}^{6} ! 6$ & 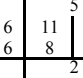 & $\frac{10}{7}$ & 9 & ${ }_{28}$ & ${ }^{41}$ & $N_{s}$ & & h & (1) \\
\hline 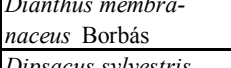 & -1 & 1 & : & $:$ & !: & & i & - & i: & & : & 11 & 1 & i: & $\left.\right|^{2} \mid$ & 1 & 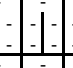 & $1:$ & $1:$ & $: \frac{3}{2} \sqrt[3]{\frac{3}{2}}$ & 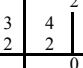 & ${ }_{2}^{4}$ & ${ }^{4}$ & ${ }^{12}$ & ${ }_{14}$ & $N_{s}$ & & ${ }^{\mathrm{h}}$ & (1) \\
\hline 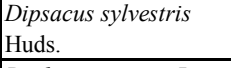 & $=1$ & 1 & : & & L: & & i & & : & & : & : : & i: 1 & $:=1$ & : & 1 & & : & : & 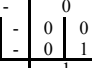 & $\begin{array}{llll} & 0 \\
\end{array}$ & i & 1 & 1 & 1 & $H a$ & & th & \\
\hline Draba nemorosa L. & & : & $1:$ & & :: : : & . & $\therefore$ & $: 1$ & : & & : & $\left|\frac{2}{2}\right|:=$ & 1: $: 1$ & $D=1$ & I & : & 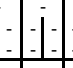 & 涑: & $1:$ il & $\left|:=\frac{2}{2}\right|_{0}^{1}$ & $\begin{array}{lll}1 & 1 \\
0 & 3 \\
3 & 3 \\
\end{array}$ & $\begin{array}{c}1 \\
0 \\
0\end{array}$ & 5 & 6 & 8 & $N_{s}$ & & $\mathrm{i}$ & \\
\hline chinops & & ili & $\because$ & & $i: \frac{1}{2} \quad$ & it & j. & - & : & & i: & iili: & : & & & & 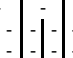 & : : : & $\therefore:=1$ & $|:| \begin{array}{ll}0 \\
\left.{ }_{3}^{0}\right|_{4} ^{2}\end{array}$ & \begin{tabular}{l|l|l|l|l|l}
2 & 0 \\
0 & 0
\end{tabular} & ${ }_{6}^{2}$ & ${ }^{4}$ & 10 & ${ }_{14}$ & $\mathrm{Ha}$ & & ${ }^{\mathrm{h}}$ & Artemi \\
\hline Echium vulgare $\mathrm{L}$. & & & i. & & ii i: & & & & : & & & ini & i & & & & i: & & & {$\left[\begin{array}{cc}0 \\
0 \\
13\end{array}\right]_{14}^{3}$} & $\begin{array}{lll}3 & 0 \\
14 & 0 \\
16\end{array}$ & $\begin{array}{l}3 \\
16 \\
\end{array}$ & 19 & ${ }^{30}$ & ${ }_{35}$ & Ap & & $\begin{array}{l}\mathrm{t} \\
\mathrm{h} \\
\end{array}$ & Artemii \\
\hline \begin{tabular}{|l} 
Echinochloa crus-gall \\
(L.). Beeauv.
\end{tabular} & & is & & & $1:$ & & -1 & & : & & : & 1 & D: & & & & & : & $:=1$ & $\begin{array}{lll}0 & 0 \\
0 & 1 & \\
0 & \end{array}$ & \begin{tabular}{c|c|c|}
0 & 0 \\
1 & 0 & 0 \\
\end{tabular} & $\begin{array}{c}0 \\
1 \\
\end{array}$ & 1 & 1 & 1 & $A r$ & 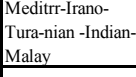 & t & SSel nedi \\
\hline $\begin{array}{l}\text { Elisanthe viscosa (L.) } \\
\text { Rupr. }\end{array}$ & 1 & 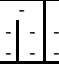 & $\therefore$ & : & : & : & i: & & i & & : & ii: & : & $=1$ & 121- & & : i: & i: & \begin{tabular}{|l|l}
2 & 2 \\
2 & 2 \\
2 & 2
\end{tabular}$=1$ & {$\left[\begin{array}{lll}2 \\
5\end{array}\right]_{1}^{3}$} & \begin{tabular}{|l|l|l|}
3 & 3 \\
1 & 1 \\
\end{tabular} & $\begin{array}{ll}4 \\
1\end{array}$ & 8 & 12 & 17 & $N_{s}$ & & h & \\
\hline
\end{tabular}




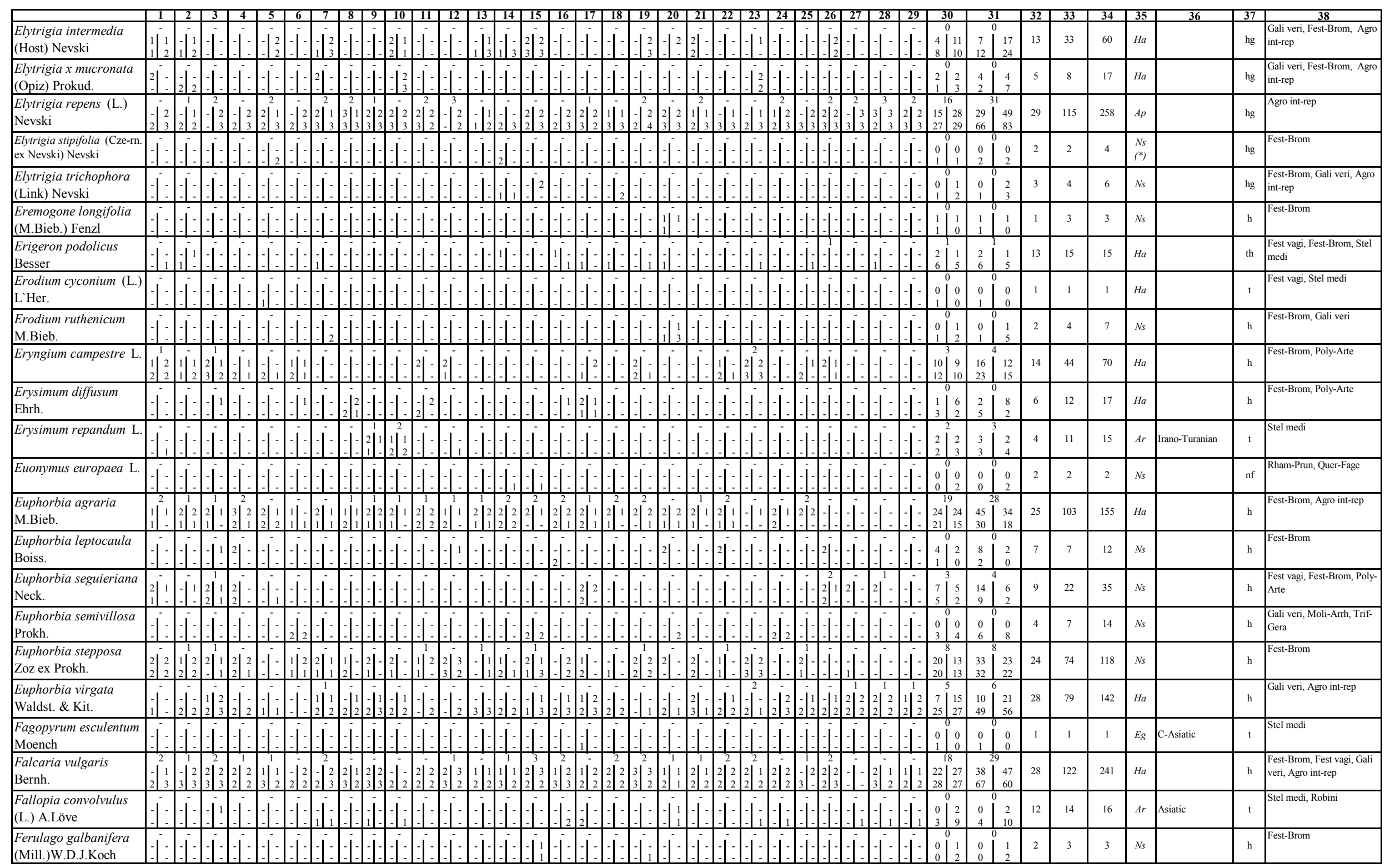




\begin{tabular}{|c|c|c|c|c|c|c|c|c|c|c|c|c|c|c|c|c|c|c|c|c|c|c|c|c|c|c|c|c|}
\hline & & & & & & & 10 & & & & & & & & & & & & & 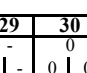 & 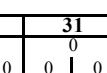 & \begin{tabular}{|l|l|} 
& 32 \\
& 32 \\
\end{tabular} & \begin{tabular}{|l|l|}
33 \\
3
\end{tabular} & & & 36 & & 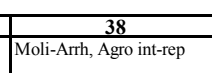 \\
\hline Huds. & : & & $1-1$ & 1 & & & : & & & & & & 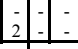 & & & & & & & $\left|:=\begin{array}{ll}0 \\
0 \\
0\end{array}\right|$ & & \begin{tabular}{l|l}
0 & 3 \\
& \\
\end{tabular} & ${ }^{3}$ & & ${ }^{\mathrm{Ha}}$ & & & \\
\hline 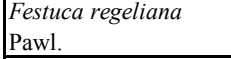 & & & & -1 & & & -1 & & & & & & 1 & & & 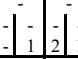 & & & : & 青 & \begin{tabular}{|l|l|l|l|l|l|l|l|l}
0 & 0 \\
2 & 3 & 3
\end{tabular} & \begin{tabular}{l|l|}
0 & 3 \\
\end{tabular} & ${ }^{4}$ & 5 & ${ }^{s s}$ & & & Aste trip, Alth offi \\
\hline $\begin{array}{l}\text { Festura rupicola } \\
\text { Hentf. }\end{array}$ & 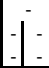 & & & & & & : & & & & & & : & & & 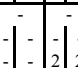 & & & is & & $\begin{array}{l}2 \\
9\end{array}$ & \begin{tabular}{|l|l|}
3 & 10 \\
14 & \\
\end{tabular} & 16 & 28 & $N_{s}$ & & $\mathrm{~h}$ & Fest-Brom, Gail ven \\
\hline $\begin{array}{l}\begin{array}{l}\text { Festuca valesiaca } \\
\text { Gaudin }\end{array} \\
\text { a }\end{array}$ & | & & 型 & 年 & & 3 & 3 & & & & & & $3^{3} 3^{3} 3^{3}$ & & & & & & $\begin{array}{lll}2 & 2 \\
3 & 3 \\
2 & 2 \\
2 & 2\end{array}$ & {$\left[\begin{array}{cc}280 \\
28 \\
18\end{array}\right.$} & 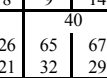 & \begin{tabular}{|l|l|}
67 & 29 \\
29 &
\end{tabular} & ${ }_{113}$ & 233 & ${ }^{N s}$ & & & \\
\hline $\begin{array}{l}\text { Ficaria calthifolia } \\
\text { Rchb. }\end{array}$ & 1 & & 1 & 1: & & & 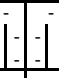 & & & & & & 1 & & & $\therefore$ & & & 1 & 10 & \begin{tabular}{l|l|l|l}
2 & 2 & 2 \\
1 & 1 & 3 \\
1
\end{tabular} & \begin{tabular}{|l|l}
2 & 2 \\
3 &
\end{tabular} & $s$ & 8 & ${ }_{\text {ss }}$ & & gh & \\
\hline $\begin{array}{l}\text { Filipendula vulgaris } \\
\text { Moench }\end{array}$ & il & & L: & -1 & & & 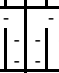 & & & & & & 1 & & & $=1$ & & 1 & $\therefore$ & 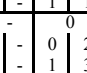 & $\begin{array}{lll}1 & 3 \\
3 & 0 & 2 \\
1 & 2 & 6\end{array}$ & \begin{tabular}{l|l}
3 & 3 \\
\end{tabular} & 6 & 9 & $N_{s}$ & & hg & Moli-Arht, Gail veri \\
\hline 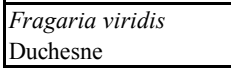 & 2 & 这| & -1 & 1 & : & $: 1:$ & 1 & & $: 1$ & & & : & $i_{2}=i_{2}$ & 12 & : & $1: 1$ & & & $1:=$ & $\left|\begin{array}{l}1 \\
2\end{array}\right|$ & 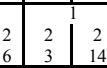 & \begin{tabular}{c|c}
2 & 6 \\
14
\end{tabular} & 12 & 22 & $N_{s s}$ & & & 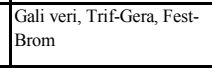 \\
\hline Fraxinus excelsior L. & : & & 1 & i & & & 1 & & & & & & : : & $-i$ & $j$ & : & & & $\therefore$ & $\mid \begin{array}{lll}0 \\
0 \\
1\end{array}$ & \begin{tabular}{l|ll}
0 & 0 \\
0 & 0 & 0 \\
1 & 0
\end{tabular} & \begin{tabular}{l|l}
0 \\
0 & 1 \\
\end{tabular} & 1 & 1 & $N_{s}$ & & $\mathrm{mt}^{\mathrm{m}}$ & Salipur, Robin \\
\hline $\begin{array}{l}\begin{array}{l}\text { Fraxinus pennsylva- } \\
\text { nica Marschall }\end{array} \\
\end{array}$ & 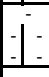 & & 1 & 1 & - & & 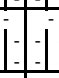 & & & & & & i: & -1 & 1 & i: & & i & $=$ & $\begin{array}{ll}0 \\
0 \\
3 & 0 \\
3 & 0 \\
\end{array}$ & \begin{tabular}{l|l|l}
0 & 0 \\
0 & 0 & 0 \\
3 & 3 & 3 \\
3
\end{tabular} & \begin{tabular}{l|l}
0 \\
\\
\end{tabular} & 6 & 6 & Ke & North American & & Resoin \\
\hline $\begin{array}{l}\begin{array}{l}\text { Fumaria schleicheri } \\
\text { Soy.-Willem. }\end{array} \\
\end{array}$ & 1 & & 1 & : & & & 1 & & & & & 1 & $1:$ & 1 & $1:$ & $=$ & & 1 & 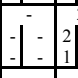 & $\begin{array}{lll}2 & 1 \\
2 & 1 \\
\end{array}$ & $\begin{array}{llll}1 & 2 & 2 \\
1 & 2 & 2 \\
1 & 4\end{array}$ & \begin{tabular}{|l|l|}
2 & 3 \\
\end{tabular} & 7 & ${ }^{11}$ & ${ }_{A r}$ & trano-Tunamin & & Sterleneit \\
\hline \begin{tabular}{|l|} 
Fimmaria vaillantii \\
Loisel.
\end{tabular} & : & & $:=1$ & $:$ & & & $\vdots$ & & & & & & : & $\mathrm{i} i \mathrm{z}_{2}$ & : & 1 & & I: & $i:=1$ & \begin{tabular}{lll}
4 \\
\hdashline \\
3 \\
3
\end{tabular} & $\begin{array}{ll}2 \\
2 \\
\frac{5}{3} \sqrt[3]{2}\end{array}$ & ${ }^{10}$ & 16 & 18 & ${ }_{A r} r$ & trano-Turar-ian & $\mathrm{t}$ & Situl madi \\
\hline Fumaria sp. & 1 & & $:=1$ & 1 & 1 & & $=$ & & & & & & 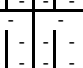 & 1 & : & j: & & $\mathrm{H} \mathrm{I}_{2}$ & 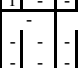 & 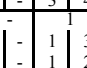 & $\begin{array}{lll}3 & 1 & 3 \\
3 & 1 & 3 \\
3\end{array}$ & \begin{tabular}{l|l}
5 \\
3 \\
3 & 4
\end{tabular} & 8 & 9 & ${ }_{A r}$ & & t & Stelinnait \\
\hline $\begin{array}{l}\text { Gagea er erbescens (Bes- } \\
\text { ser) Schult. \& Schult.f. }\end{array}$ & 1 & & 1 & 1 & -1 & & $\vdots$ & & & & & & il: & -1 & i & $=$ & & 1 & : & 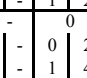 & 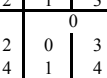 & s & 7 & 8 & ${ }_{N s}$ & & $\mathrm{~g}$ & \\
\hline 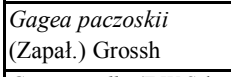 & 1 & & 1 & 1 & : & & 1 & & & & & & i: & 1.1.1. & i & 1 & & : & $=$ & $\begin{array}{l}5 \\
2\end{array}$ & \begin{tabular}{ll|l}
2 & 6 \\
7 & 6 & 2 \\
3 & 7
\end{tabular} & \begin{tabular}{|l|l|}
2 & 10 \\
& 10
\end{tabular} & 17 & 19 & ${ }_{N s}$ & & $\mathrm{~g}$ & \\
\hline 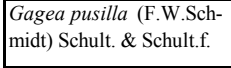 & 1 & & $D_{1}^{1}=12$ & $:$ & 11 & & $i$ & & : & & & & Di: : : & : & & $:=$ & & & 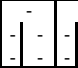 & {$\left[\begin{array}{lll}0 & = & 0 \\
0 & 1 \\
0 & 1\end{array}\right.$} & 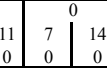 & $\begin{array}{ll}14 & 12 \\
0 & 12\end{array}$ & 15 & 21 & ${ }^{s s}$ & & $\mathrm{~g}$ & Ste-Brom, \\
\hline 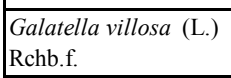 & 1 & & 2 & | & -1 & & 1 & & & & & & $: 1$ & 11 & $1:$ & $=1$ & 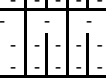 & & : & $\left.\mid \begin{array}{lll}3 \\
2\end{array}\right]$ & \begin{tabular}{l|l|l|l}
4 & 5 & 6 \\
3 & 3 & 6
\end{tabular} & \begin{tabular}{l|l|}
6 & 4 \\
\end{tabular} & 12 & 18 & $\mathrm{Ns}_{\mathrm{s}}$ & & $\mathrm{h}$ & Fest-Brom \\
\hline \begin{tabular}{|l} 
Galium aparine $\mathrm{L}$. \\
\end{tabular} & $2^{2}$ & & is & a & & & i: & & & & & & & & & & & & 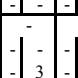 & $\begin{array}{lll}2 & 1 \\
1 & 14 \\
2 & 2 & 2 \\
7 & 2\end{array}$ & $\begin{array}{ll}0 \\
6 \\
62 & 19 \\
13 & 9 \\
39\end{array}$ & 25 & 49 & 82 & $A p$ & & $t$ & Gailutur, R \\
\hline \begin{tabular}{|l|} 
Galium humifusum \\
n.Bieb.
\end{tabular} & 1 & & 1 & | & & & $\therefore$ & & & & & & & 1 & & & & & 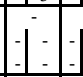 & $\begin{array}{lll}0 & 0 \\
0 & 0\end{array}$ & \begin{tabular}{l|l}
0 \\
0 \\
0 & 1 \\
0 & 1 \\
0
\end{tabular} & 1 & 1 & 1 & Ha & & $\mathrm{h}$ & $\begin{array}{l}\substack{\text { calin veri, } \\
\text { Bomm }} \\
\text { tom }\end{array}$ \\
\hline $\begin{array}{l}\text { Galium ruthenicum } \\
\text { will. }\end{array}$ & : & & 1 & 1 & -1 & & : & & & & & & | & 1 & & $: 1$ & & & i: & $|:| \begin{array}{l}2 \\
5\end{array} \mid$ & \begin{tabular}{c|c}
3 & 2 \\
10 & 4
\end{tabular} & \begin{tabular}{|l|l|}
2 & 6 \\
\end{tabular} & 11 & 20 & $N_{s}$ & & $\mathrm{~h}$ & Fest-Brom \\
\hline Galium spurium L. & ii & & & 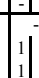 & & & -1 & & & & & & & & & & & & $\therefore$ & $\frac{1}{2}$ & $\begin{array}{l}111 \\
38 \\
8\end{array}$ & 9 & 18 & 29 & ${ }_{A r}$ & 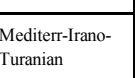 & $t$ & Stel medi \\
\hline Galium verum L. & 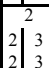 & & $\mid \begin{array}{l}2 \\
3\end{array}$ & $\mid \begin{array}{l}2 \\
2\end{array}$ & 1 & & $2^{2}$ & & & 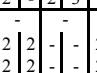 & $\frac{1}{2}$ & ia: & $a^{3} \frac{2}{3} 2_{3}^{3}$ & $-3^{2}$ & $i:$ & : & 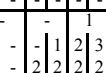 & 1 & $\therefore$ & $\mid \begin{array}{ll}1 & 1 \\
14 & 14 \\
18 & 1\end{array}$ & 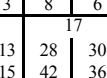 & $\begin{array}{ll}60 \\
30 \\
30\end{array}$ & ${ }_{71}$ & 153 & ${ }_{N s}$ & & $\mathrm{~h}$ & Gali veri \\
\hline $\mid \begin{array}{l}\text { Galium vollynicum } \\
\text { Pooded. }\end{array}$ & 1 & & is r a & . & 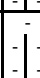 & $i$ & $\mid \frac{2}{2}$ & & & & & 1 & $a^{1}$ & i: & 年 1 & $=1$ & & & $\therefore$ & 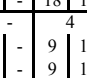 & 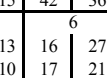 & \begin{tabular}{l|l|}
27 & 15 \\
21 & \\
2
\end{tabular} & 45 & 87 & \begin{tabular}{|l}
$s s$ \\
(ns)
\end{tabular} & & hg & ext-Brom \\
\hline eranium sanguineum & -1 & & 1 & -1 & & & j. & & & & & & & & & $j$ & j & & $\therefore$ & $=0$. & 010 & \begin{tabular}{|l|l|}
0 & 1 \\
0
\end{tabular} & 2 & 2 & $N_{s}$ & & h & Trif-Gera \\
\hline
\end{tabular}




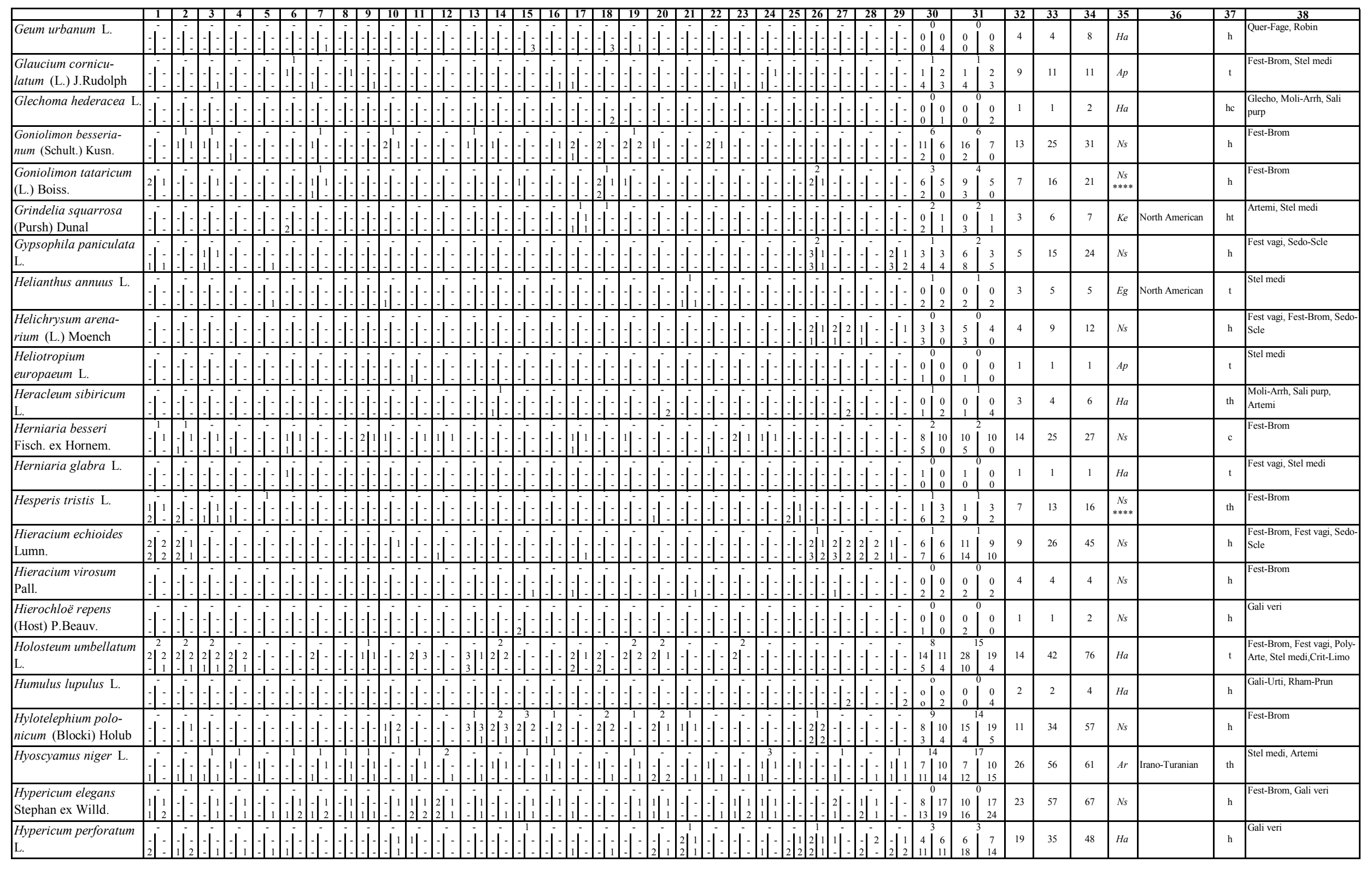




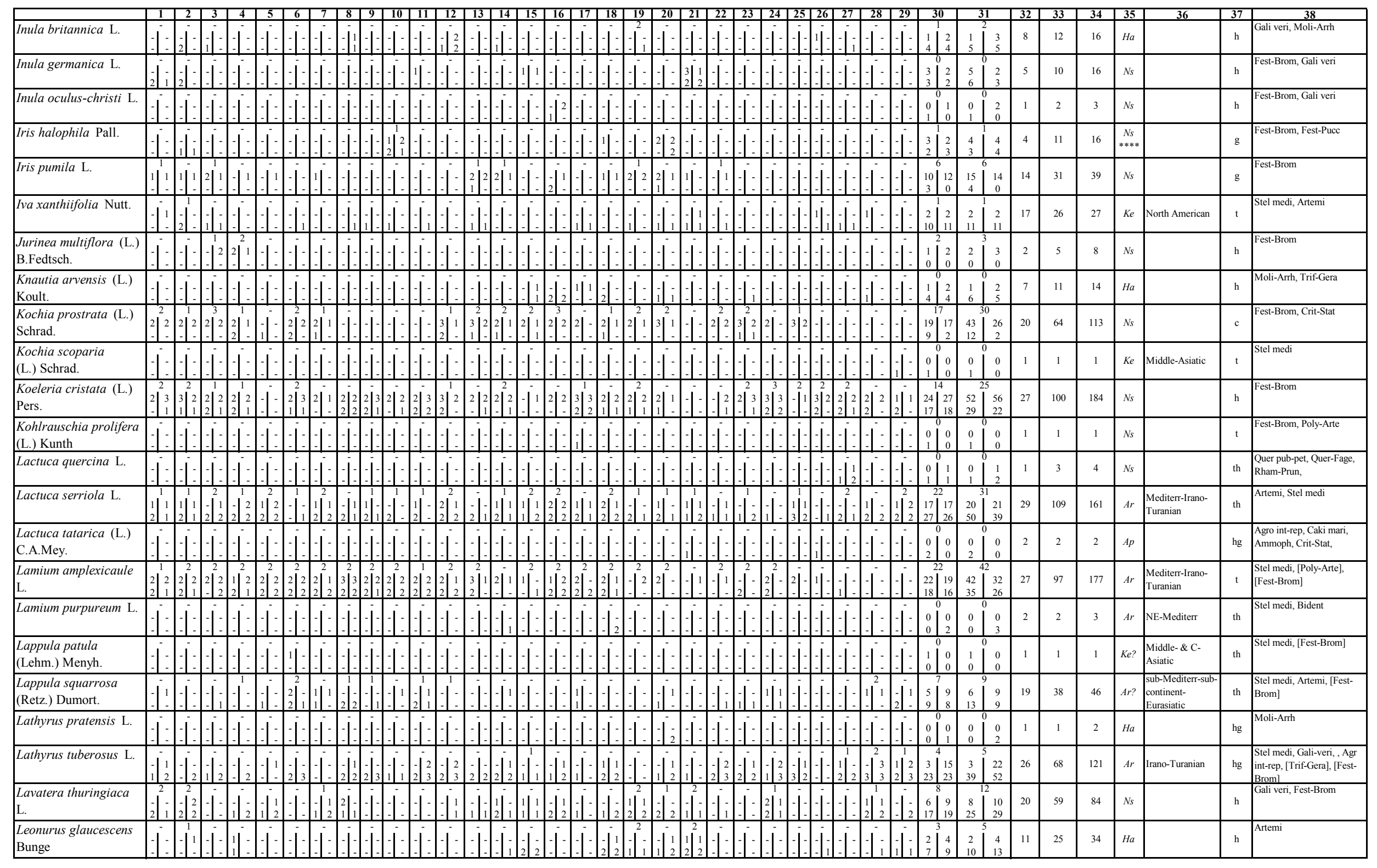




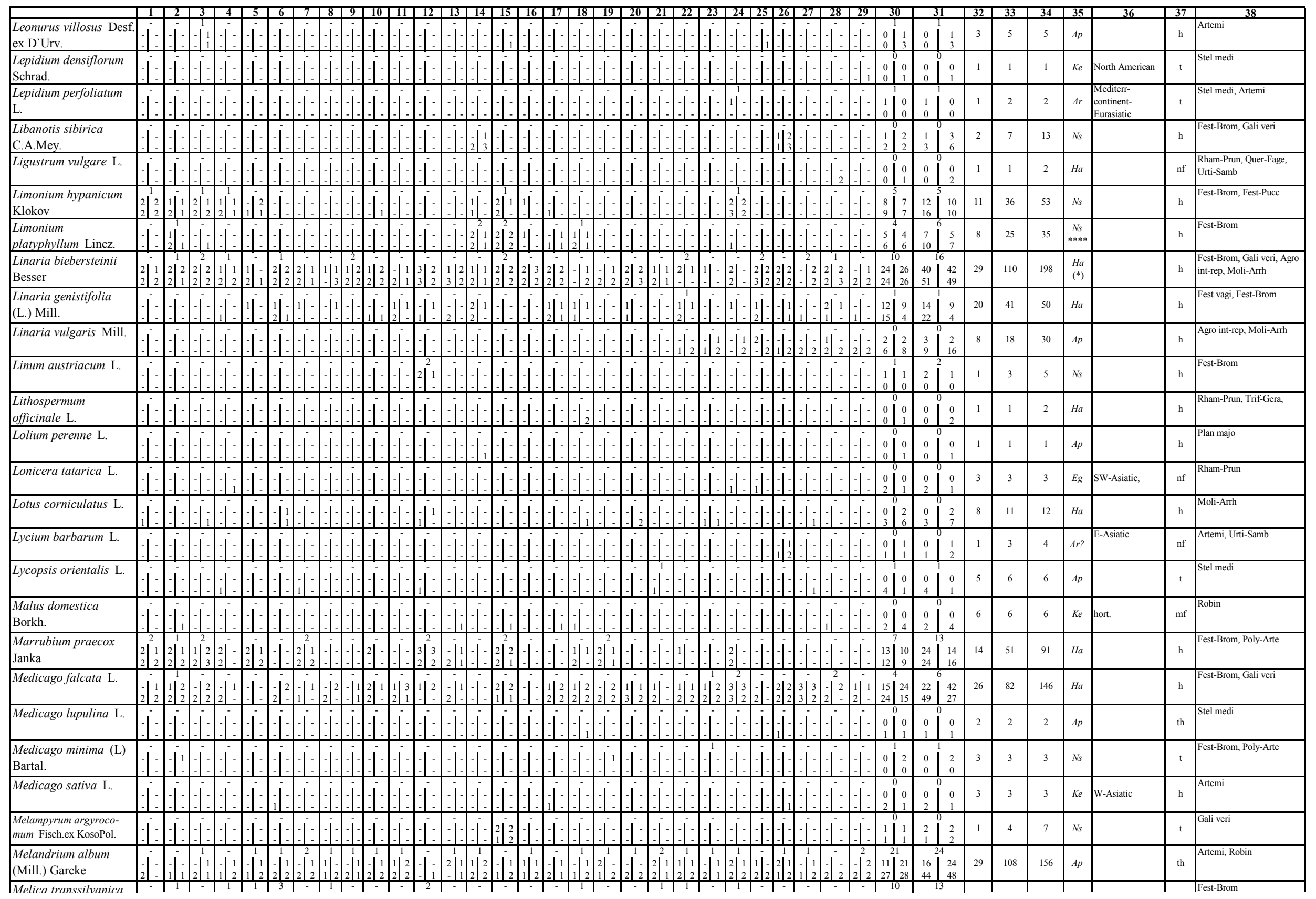




\begin{tabular}{|c|c|c|c|c|c|c|c|c|c|c|c|c|c|c|c|c|c|c|c|c|c|c|c|c|c|c|c|c|c|c|c|c|c|c|c|c|}
\hline & & & & & 0 & & 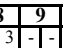 & 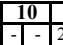 & $\frac{12}{-1}$ & \begin{tabular}{|l|l|}
2 & 13 \\
12 & -1
\end{tabular} & & $|2| 1$ & & 11 & & & $\left|\frac{20}{20}\right|$ & 4 & 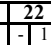 & 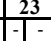 & -4 & & 21 & 0 & 429 & & & \begin{tabular}{l|l|l|l|l|}
7 & 22 \\
7
\end{tabular} & \begin{tabular}{l|l}
32 \\
2 & 21
\end{tabular} & & \begin{tabular}{l|l}
33 & \\
70 &
\end{tabular} & \begin{tabular}{|l|}
34 \\
113
\end{tabular} & \begin{tabular}{|l|}
$\mathbf{3 5}$ \\
$N s$ \\
\end{tabular} & 36 & $\frac{37}{\mathrm{hg}}$ & 38 \\
\hline Schur & & & 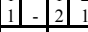 & $\begin{array}{ll}2 & 1\end{array}$ & \begin{tabular}{|c|c|c|c|}
2 & 1 & -1 \\
\end{tabular} & $\begin{array}{lll}1 & 2 & 3 \\
\end{array}$ & 3. & & 1] & \begin{tabular}{|l|l|} 
& 1 \\
\end{tabular} & 2 & $\begin{array}{lll}2 & 1 \\
\end{array}$ & $|1|^{-}$ & $1 \mid-$ & & & \begin{tabular}{|l|l|l|}
2 & 2 \\
\end{tabular} & 2 & 2 & & & & -1. & & & \begin{tabular}{l|l}
11 & 14 \\
19 & 16 \\
\end{tabular} & \begin{tabular}{l|l}
16 & 11 \\
16 & 33 \\
\end{tabular} & \begin{tabular}{l|l}
3 & 28 \\
\end{tabular} & & & & & & & & \\
\hline Melilotus albus Medik. & & & & & & & & & & 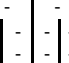 & & & & & & & $1-1$ & & & & & & -1 & & & \begin{tabular}{l|l}
0 & 0 \\
0 & 1 \\
1 & 3
\end{tabular} & $\begin{array}{l}0 \\
1\end{array}$ & $\begin{array}{l}1 \\
5\end{array}$ & 4 & & 5 & 7 & ${ }^{A p}$ & & th & Artemi \\
\hline $\begin{array}{l}\text { Melilotus officinalis } \\
\text { (L.) Pall. }\end{array}$ & & & & & & & & & & \begin{tabular}{|l|l|}
1 & -1 \\
- & -1 \\
\end{tabular} & & & & & & & & & i & & & & $\left|\begin{array}{lll}1 & 2 \\
1 & 1 \\
1\end{array}\right|^{2}$ & $\begin{array}{l}1 \\
1 \\
\end{array}$ & $-1=$ & \begin{tabular}{|l|l|l|}
2 & 11 & 13 \\
16 & 17
\end{tabular} & \begin{tabular}{l|l}
13 & 12 \\
17 & 19
\end{tabular} & $\begin{array}{l}14 \\
20\end{array}$ & 23 & & 62 & 71 & $A p$ & & th & Artemi \\
\hline $\begin{array}{l}\text { Meniocus linifolius } \\
\text { (Stephan ex Willd.) DC. }\end{array}$ & & & & & & & & & & & & -1 & & 1 & & & -1 & 1 & 10 & & & & $1:$ & & -1 & \begin{tabular}{|l|l|l|} 
& 0 \\
1 & 0 & 0 \\
1 & 0
\end{tabular} & $\begin{array}{l}1 \\
2\end{array}$ & $\begin{array}{l}0 \\
0\end{array}$ & 1 & & 2 & 3 & Ns & & $\mathrm{t}$ & Fest-Brom \\
\hline Morus alba $\mathrm{L}$. & & & & & & & & & & $:-1$ & & $1:$ & & 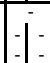 & & & $-1:$ & $1:$ & 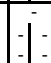 & 1 & $\therefore i$ & & $\frac{-i}{2}$ & & $-1:$ & \begin{tabular}{|l|l|} 
& 0 \\
0 & 0 \\
1 & 2
\end{tabular} & $\begin{array}{l}0 \\
2\end{array}$ & $\begin{array}{l}0 \\
2\end{array}$ & 2 & & 3 & 4 & $\mathrm{Ke}$ & E-Asiatic & $\mathrm{mf}$ & Robin \\
\hline $\begin{array}{l}\text { Muscari neglectum } \\
\text { Guss. ex Ten. } \\
\end{array}$ & L: & & & & $\therefore$ & & & 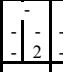 & & 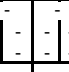 & & $\therefore$ & $\therefore:$ & $1:$ & $1:$ & & 1 & $\begin{array}{ll}2 & 1 \\
2 & 1 \\
\end{array}$ & 1 & $-1:$ & $1:$ & & 1 & & -1 & \begin{tabular}{|l|l|} 
& 0 \\
1 & 1 \\
0 & 2 \\
\end{tabular} & $\begin{array}{l}2 \\
0 \\
\end{array}$ & $\begin{array}{l}1 \\
4 \\
\end{array}$ & 2 & & 4 & 7 & Ns & & $\mathrm{g}$ & $\begin{array}{l}\text { Fest-Pucc, Gali veri, Fest- } \\
\text { Brom }\end{array}$ \\
\hline $\begin{array}{l}\text { Myosotis micrantha } \\
\text { Pall. ex Lehm. }\end{array}$ & 1 & & & $-1:-$ & & & & -2 & & -1 & & 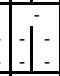 & -1 & $i$ & & $i$ & -1 & -1 & 1 & -1 & -1 & & 1 & 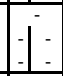 & $-1-$ & \begin{tabular}{|l|l|} 
& 0 \\
0 & 2 \\
1 & 0 \\
\end{tabular} & $\begin{array}{l}0 \\
2 \\
\end{array}$ & $\begin{array}{l}3 \\
0 \\
\end{array}$ & 3 & & 3 & 5 & $\mathrm{Ha}$ & & $\mathrm{t}$ & $\begin{array}{l}\text { Fest vagi, Fest-Brom, Poly- } \\
\text { Arte }\end{array}$ \\
\hline $\begin{array}{l}\text { Myosoton aquaticum } \\
\text { (L.) Moench }\end{array}$ & & & & & & & & & & 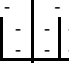 & & :- & -1. & $1-$ & & & $1:-1$ & $1:$ & 1 & & :- & & $1:$ & & $\therefore-$ & \begin{tabular}{|l|l|} 
& 0 \\
0 & 0 \\
0 & 1 \\
\end{tabular} & & $\begin{array}{l}0 \\
1 \\
\end{array}$ & 1 & & 1 & 1 & $\mathrm{Ha}$ & & gh & Bident, Sali purp, Alne glut \\
\hline Nepeta cataria $\mathrm{L}$. & & & & & & & & & & $-i$ & & $1:$ & & $\therefore$ & & & $1:$ & $-1-$ & 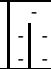 & & $\therefore$ & & 1 & & -1 & \begin{tabular}{|l|l|l|} 
& 0 \\
0 & 0 \\
0 & 2 \\
\end{tabular} & $\begin{array}{l}0 \\
0\end{array}$ & $\begin{array}{l}0 \\
2\end{array}$ & 2 & & 2 & 2 & $A r$ & E-Mediterr & ch & \begin{tabular}{|l} 
Artemi, Urti-Samb, Rham- \\
Prun
\end{tabular} \\
\hline Nepeta pannonica $\mathrm{L}$. & & & & \begin{tabular}{|l|l|} 
& 1 \\
2 & 2 \\
\end{tabular} & & & & & & -1 & & \begin{tabular}{|l|l|l|} 
& 1 \\
& 3 \\
3 & 3 \\
\end{tabular} & -1 & $1:$ & $1:$ & 1 & $\because=$ & $1:$ & 1 & 1 & $\therefore$ & & 1 & $1:$ & $\therefore$ & \begin{tabular}{|l|l|l|}
1 & 4 \\
5 & 5 \\
\end{tabular} & $\begin{array}{l}2 \\
10 \\
\end{array}$ & $\begin{array}{c}6 \\
12 \\
\end{array}$ & 6 & & 16 & 31 & Ns & & ch & Fest-Brom, Gali-veri \\
\hline $\begin{array}{l}\text { Nepeta parviflora } \\
\text { M.Bieb. }\end{array}$ & & -1 & & 1 & & & & & & -1 & & 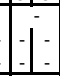 & -1 & -1 & -1 & & $-1-1$ & -1 & -1 & -1 & $-1-1$ & & 1 & & $-1-1$ & \begin{tabular}{|l|l|} 
& 1 \\
3 & 2 \\
2 & 0 \\
\end{tabular} & $\begin{array}{l}5 \\
3 \\
\end{array}$ & $\begin{array}{l}2 \\
0 \\
\end{array}$ & 6 & & 8 & 11 & Ns & & $\mathrm{h}$ & Fest-Brom \\
\hline Nigella arvensis L. & & & & & & & & & & \begin{tabular}{|l|l|} 
& $-j$ \\
1 & -1 \\
\end{tabular} & & $-1:$ & $1:$ & 年 & & & $\begin{array}{ll} \\
2\end{array}$ & $1:$ & il: & -1 & \begin{tabular}{|l|l|}
1 & 1 \\
3 & 2 \\
\end{tabular} & & $1-1$ & & $-1=$ & \begin{tabular}{|c|c|} 
& 5 \\
8 & 5 \\
12 & 7 \\
\end{tabular} & $\begin{array}{r}10 \\
21 \\
\end{array}$ & $\begin{array}{r}6 \\
9 \\
\end{array}$ & 16 & 37 & 37 & 53 & $A r$ & Balkan-W-Asiatic & $\mathrm{t}$ & Stel medi, [Fest-Brom] \\
\hline Nonea rossica Steven & & & \begin{tabular}{|l|l|l} 
& 1 & 1 \\
2 & 1 & 2 \\
1 & 1 & 1 \\
\end{tabular} & $\begin{array}{l}i \\
i \\
1\end{array}$ & & & - & & & \begin{tabular}{|l|l|} 
& $-j$ \\
1 & -1 \\
\end{tabular} & & & $\left.\begin{array}{l}1 \\
2\end{array}\right]$ & \begin{tabular}{|l|l|}
2 & - \\
2 & 2 \\
\end{tabular} & & \begin{tabular}{|l|l|} 
& 1 \\
& 1 \\
1 & 1 \\
\end{tabular} & i. & 1 & il & 1 & \begin{tabular}{|l|l|}
1 & - \\
1 & - \\
\end{tabular} & & \begin{tabular}{|l|l|l|} 
& 1 \\
1 & 1 \\
1
\end{tabular} & \begin{tabular}{|l|l|} 
& 1 \\
1 & 1 \\
\end{tabular} & $\begin{array}{r}-1 \\
1 \\
\end{array}$ & \begin{tabular}{|c|c|c|} 
& 3 & 6 \\
1 & 9 & 6 \\
13 & 10 \\
\end{tabular} & $\begin{array}{l}11 \\
17 \\
\end{array}$ & $\begin{array}{r}6 \\
11 \\
\end{array}$ & 15 & 41 & 41 & 48 & $\mathrm{Ha}$ & & $\mathrm{h}$ & Fest-Brom, Artemi \\
\hline $\begin{array}{l}\begin{array}{l}\text { Oberna cserei } \\
\text { (Baumg.) Ikonn. }\end{array} \\
\end{array}$ & 1 & & & $1:-1$ & & \begin{tabular}{|l|l|} 
& 1 \\
\end{tabular} & 资 & & 1 & $:-1$ & & $-1:$ & $\begin{array}{ll}1 \\
2 \\
2 & \text { i } \\
\end{array}$ & 1 & : & $1:$ & $-1:$ & $1:$ & $1=$ & : & $-1:$ & -1 & $1:=$ & $1:$ & -1 & \begin{tabular}{|l|l|} 
& 1 \\
2 & 1 \\
& 2 \\
\end{tabular} & $\begin{array}{l}2 \\
4 \\
\end{array}$ & $\begin{array}{l}1 \\
2 \\
\end{array}$ & 6 & & 9 & 10 & $\mathrm{Ha}$ & & $\mathrm{h}$ & Fest-Brom, Artemi \\
\hline $\begin{array}{l}\text { Onobrychis viciifolia } \\
\text { Scop. }\end{array}$ & -1 & $\therefore$ & $\therefore:$ & $::$ & $:$ & $\therefore:$ & : & $1:$ & 1 & $:: 1$ & & $\therefore:$ & $:-1$ & i & $:-$ & : & 1 & 1 & $:=$ & $1:$ & $:=$ & & $1:$ & $1:$ & $:-:$ & \begin{tabular}{|l|l|}
0 & 0 \\
1 & 1 \\
\end{tabular} & $\begin{array}{l}0 \\
2 \\
\end{array}$ & $\begin{array}{l}0 \\
1 \\
\end{array}$ & 1 & & 2 & 3 & $\mathrm{Ke}$ & \begin{tabular}{|l|}
$\begin{array}{l}\text { Mediterr-Irano- } \\
\text { Turanian }\end{array}$ \\
\end{tabular} & $\mathrm{h}$ & \begin{tabular}{|l} 
Trif-Gera, Moli-Arrh, Stel \\
medi
\end{tabular} \\
\hline $\begin{array}{l}\text { Onopordum acanthium } \\
\text { L. } \\
\end{array}$ & 1 & il & & $\begin{array}{ll} & 1 \\
1 & 1 \\
\end{array}$ & & & il & & & $\left|\begin{array}{ll}i & - \\
1\end{array}\right|$ & & 11. & -1 & 2 & -1 & i. & i & -1 & il: & i & $\begin{array}{ll}-1 \\
1\end{array}$ & & $1=$ & i & $-1:$ & \begin{tabular}{|c|c|}
4 & 6 \\
19 & 11 \\
\end{tabular} & \begin{tabular}{c|c}
6 & 5 \\
11 & 20 \\
11
\end{tabular} & $\begin{array}{r}6 \\
11 \\
\end{array}$ & 24 & 48 & 48 & 50 & ${ }_{A r}$ & \begin{tabular}{|l} 
Mediterr-Irano- \\
Turanian
\end{tabular} & $\mathrm{h}$ & \begin{tabular}{|l|l} 
Artemi \\
\end{tabular} \\
\hline $\begin{array}{l}\text { Ornithogalum kochii } \\
\text { Parl. } \\
\end{array}$ & $i$ & & & & $1:$ & & & & & $\mid$\begin{tabular}{l|l}
1 & 1 \\
1 & 1
\end{tabular} & & & $-1-1$ & -1 & -1 & & & & -11 & & -1 & & 1 & & -1 & \begin{tabular}{|l|l|} 
& 0 \\
2 & 4 \\
0 & 2 \\
\end{tabular} & $\begin{array}{l}2 \\
0 \\
\end{array}$ & $\begin{array}{r}5 \\
2 \\
\end{array}$ & 6 & & 8 & 9 & \begin{tabular}{|c|}
$N s$ \\
$* * * * *$ \\
\end{tabular} & & $\mathrm{~g}$ & Fest-Brom \\
\hline $\begin{array}{l}\text { Otites densiflorum } \\
\text { (D'Urv.) Grossh. aggr. }\end{array}$ & \begin{tabular}{|l|l|} 
& 2 \\
3 & 2 \\
1 & 1 \\
\end{tabular} & & & & & & -1 & 1.1 & 1 & 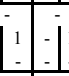 & & -1 & \begin{tabular}{|l|l|}
1 & 1 \\
2 & - \\
\end{tabular} & i 1. & $\begin{array}{l}1 \\
2\end{array}$ & & -1 & -1 & 1 & -1 & $\begin{array}{l}1 \\
1\end{array} \mid$ & & it: & & \begin{tabular}{ll|l}
1 & - \\
1 & - \\
\end{tabular} & \begin{tabular}{|l|l|}
4 & \\
12 & 10 \\
11 & 3 \\
11
\end{tabular} & $\begin{array}{l}16 \\
16 \\
\end{array}$ & $\begin{array}{r}12 \\
3 \\
\end{array}$ & 16 & 4 & 40 & 54 & $\mathrm{Ns}_{\mathrm{s}}$ & & $\mathrm{h}$ & $\begin{array}{l}\text { Fest vagi, Fest-Brom, Poly- } \\
\text { Arte }\end{array}$ \\
\hline \begin{tabular}{|l}
$\begin{array}{l}\text { Otites hellmannii } \\
\text { (Claus) Klokov }\end{array}$ \\
\end{tabular} & $-1-$ & $\therefore$ & $\begin{array}{lll} & 1 \\
\end{array}$ & $1:$ & $1:$ & & : 1 & & -1 & $1:-1$ & & 1 & F-1. & $1:$ & 1 & -1 & 1 & 1 & 1 & $1:$ & 1 & & $11: 1$ & $1:$ & $:=$ & \begin{tabular}{|l|l|l|l|l}
1 & 1 \\
0 & 0 \\
\end{tabular} & $\begin{array}{l}1 \\
0 \\
\end{array}$ & $\begin{array}{l}1 \\
0 \\
\end{array}$ & 1 & & 2 & 2 & Ns & & $\mathrm{h}$ & Fest-Brom \\
\hline $\begin{array}{l}\text { Oxytropis pilosa (L.) } \\
\text { DC. }\end{array}$ & & & & -1 & $\begin{aligned} 2 & -1 \\
-1 & -1\end{aligned}$ & & $\begin{array}{llll} & 1 \\
& 1 & 1\end{array}$ & & 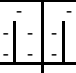 & \begin{tabular}{|l|l|}
1 & -1 \\
- & -1
\end{tabular} & & -1 & -1 & $\begin{array}{ll}1 & 2 \\
1 & 2 \\
1\end{array}$ & $1:$ & & \begin{tabular}{|l|l|}
2 & 3 \\
2 & -1 \\
\end{tabular} & 1 & 1 & 1 & $\begin{array}{ll}1 & 2 \\
-1 & 2\end{array}$ & & $\therefore$ & $i$ & 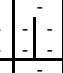 & \begin{tabular}{l|l}
4 & 8 \\
2 & 2 \\
\end{tabular} & $\begin{array}{l}4 \\
3 \\
\end{array}$ & $\begin{array}{r}13 \\
3 \\
\end{array}$ & 10 & 18 & 18 & 25 & Ns & & $\mathrm{h}$ & Fest-Brom \\
\hline Panicum miliaceum $\mathrm{L}$. & & & 10 & & $-1:-1$ & & $-1-1-1$ & & $-1-1$ & $:-1$ & & -1 & -1 & 1] & & & $\begin{array}{lll} & 1 \\
1 & \\
\end{array}$ & 1 & -1 & . & - & & -1. & $1=$ & $-1-$ & \begin{tabular}{|l|l|} 
& 0 \\
0 & 1 \\
2 & 1 \\
\end{tabular} & $\begin{array}{l}0 \\
2 \\
\end{array}$ & $\begin{array}{l}1 \\
1 \\
\end{array}$ & 3 & 4 & 4 & 4 & $\mathrm{Ke}$ & C-Asiatic & $\mathrm{t}$ & Stel medi \\
\hline Papaver dubium $\mathrm{L}$. & & & & & $1-1-1$ & & & & & & & & & & & & & & & & & & $1-1-1-$ & $i-$ & 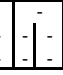 & \begin{tabular}{|l|l|} 
& 0 \\
0 & 0 \\
3 & 4 \\
\end{tabular} & & \begin{tabular}{|l|l|}
0 \\
3
\end{tabular} & 6 & & 7 & 7 & $A r$ & \begin{tabular}{|l}
$\begin{array}{l}\text { Mediterr-Irano- } \\
\text { Turanian }\end{array}$ \\
\end{tabular} & $\mathrm{t}$ & Stel medi \\
\hline
\end{tabular}




\begin{tabular}{|c|c|c|c|c|c|c|c|c|c|c|c|c|c|c|c|c|c|c|c|c|c|c|c|c|c|c|c|c|}
\hline Papaver rhoeas L. & & & & & & 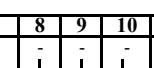 & & & & & ${ }_{18}^{18}$ & & & & & & & & & & & & & & & $\frac{36}{\text { Neditirarlano- }}$ & & Siel medi 38 \\
\hline & 1 & & & & & & & : & : : & & & & | & $1:$ & : & & 法 & & & & $\begin{array}{l}1 \\
4 \\
4\end{array}$ & 8 & 14 & 14 & & & & \\
\hline $\begin{array}{l}\begin{array}{l}\text { asstinaca c clusii } \\
\text { (Ledb.) M.Pimen. }\end{array} \\
\end{array}$ & $2^{1}$ & & & & & : & & 1 & : & 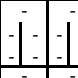 & & & & 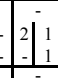 & 1 & & i: & : : & & $\left.\right|_{3} ^{8}$ & $\begin{array}{ll}189 \\
58 \\
5\end{array}$ & 9 & 30 & 49 & ${ }_{N s}$ & & ${ }^{\mathrm{h}}$ & \\
\hline 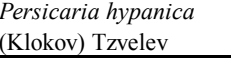 & : & & & & & & & $:$ & $: 1$ & -1 & & & $: 1$ & $1:$ & 1 & & -1 & $:$ & $: 1$ & L & 象 & 1 & 1 & 1 & Ha & & $t$ & \\
\hline Pencedanum alsaticum & : : & 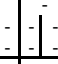 & 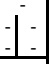 & & & : & & 1 & $21 \frac{1}{2}$ & 1 & $:$ & 尘 & 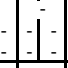 & i: & : & & : & $\therefore$ & -1 & $\perp \frac{2}{2}+x-a+2$ & 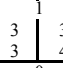 & 2 & 9 & 14 & $N_{s}$ & & $\mathrm{~h}$ & 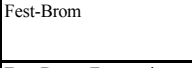 \\
\hline 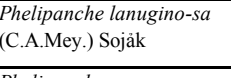 & : & 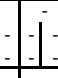 & 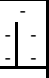 & & i & $\therefore$ & $i$ & 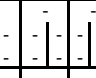 & $\therefore$ & it & $1:$ & ! & 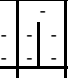 & 1 & 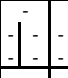 & & $i$ & $\therefore$ & 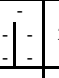 & $\left.\right|_{1} ^{2}$ & $\begin{array}{ll}2 \\
2\end{array}$ & 2 & ${ }^{6}$ & 7 & $\mathrm{ss}^{\mathrm{s}}$ & & ${ }^{8}$ & \\
\hline 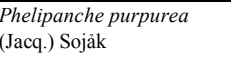 & : & 1 & 1 & & & E & & i & $\therefore$ & i & & 1 & 1 & $i$ & $V_{12}^{2}$ & & : & $\therefore$ & : & $j_{2}^{4}$ & 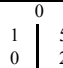 & ${ }^{4}$ & 7 & 8 & $N_{s}$ & & $\mathrm{~s}$ & Fest-Brom, Artemi \\
\hline $\begin{array}{l}\text { Phleum phleoides (L.) } \\
\text { H.Harst. }\end{array}$ & : : & : & : & & & 1: & & : & $\therefore$ & & & & : & : & : & & : & $i:$ & : & 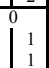 & 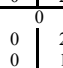 & 1 & ${ }^{2}$ & ${ }^{3}$ & ${ }^{s s}$ & & ng & ali veri, fess \\
\hline Phleum pratense $\mathrm{L}$. & : & & 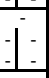 & & : & : & T. & : : & : & 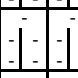 & 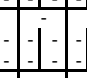 & : & : & 1 & : & & i & $\therefore$ & : & i: & $\begin{array}{lll}0 & 1 \\
0 & 1 \\
0\end{array}$ & 1 & 1 & 1 & Ha & & $\mathrm{gh}^{\mathrm{h}}$ & loli-Armh \\
\hline hlomis hybrida Zelen. & : & & I & 1 & & & & 1 & : & 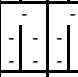 & : & 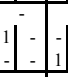 & $i: 1:$ & : & il & & $1:$ & $1:$ & a & $\left.\right|_{1} ^{2}$ & 党 & 7 & 9 & ${ }^{10}$ & 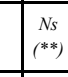 & & ${ }^{\mathrm{h}}$ & Fast-Brom \\
\hline 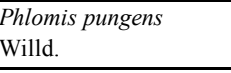 & 2 & 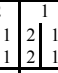 & ii & & 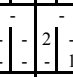 & 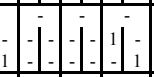 & & $\begin{array}{l}1 \\
1 \\
2\end{array}$ & $2 !$ & & & $22_{1}^{2}$ & $i_{1} \mid !$ & $1:$ & 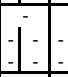 & & : & : & : & 29 & $\frac{18}{18}$ & ${ }_{14}$ & ${ }^{50}$ & 68 & ${ }_{s s}$ & & $\mathrm{~h}$ & 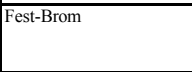 \\
\hline Phlomis tuberosa L. & 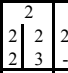 & 1 & $d_{3}^{2}$ & $\frac{1}{3}$ & & $\mid \frac{1}{2} 2^{2}$ & & $y_{2}^{1}$ & $\frac{1}{2}$ & & i|: & 迹 & $\begin{array}{ll}2 \\
2 \\
2\end{array}$ & $\left|\frac{2}{2}\right|^{2}$. & $\mathrm{i} i$ & & i: & : & 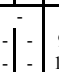 & $a_{16}^{17}$ & $\begin{array}{l}\left.15^{4}\right|^{2} \\
13\end{array}$ & 20 & ${ }_{57}$ & 105 & ${ }_{s s}$ & & hg & \\
\hline $\begin{array}{l}\text { Phraggmites australis } \\
\text { (Cav.) Trin. ex Steud. }\end{array}$ & $1:$ & 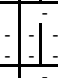 & 1 & & & 1 & & : & : & 1 & : & : & $1:$ & 1 & 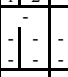 & & 1 & i & 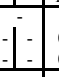 & $\prod_{1}^{0}$ & 年 & 1 & 1 & 1 & ${ }_{N s}$ & & $\mathrm{gv}$ & Phragmi \\
\hline & 1 & 1 & ii & & & & & i & : & & : & & : & i i & : & & i i & $\left|\begin{array}{ll}2 \\
2\end{array}\right| \begin{array}{l}2 \\
2\end{array}$ & $\frac{1}{1}$ & $\left.\right|_{1} ^{12}$ & $\begin{array}{ccc}5 & 0 \\
1 & 1 & 1 \\
1 & & \end{array}$ & ${ }_{17}$ & ${ }^{41}$ & 52 & Ha & & h & estraga, $A$. \\
\hline $\begin{array}{l}\text { Picrisis irida Ledeb. ex } \\
\text { Spreng. }\end{array}$ & 1 & 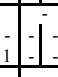 & : & & : & : & & : & : & 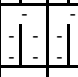 & 1 & i & 1 & : & 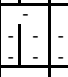 & & 1 & 1 & : & $\bigsqcup_{1}^{0}$ & $\begin{array}{ll}01 \\
01 \\
\end{array}$ & 1 & 2 & 2 & $N_{s}$ & & $\mathrm{~h}$ & Fest vagit \\
\hline & $2 \mathrm{i}$ & 1 & i: & & & E & & $1: 1$ & $: 1:$ & & : & & : & ii & 1 & & i: i: & ii & 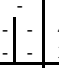 & $\varliminf_{11}^{3}$ & 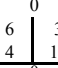 & 13 & ${ }^{20}$ & 23 & Ha & & h & \\
\hline Plantago major $\mathrm{L}$. & : & i & : & & : & $:$ & & : : : & $:$ & 1 & : & 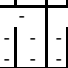 & : & : & : & & i: & : & : & $\left.\right|_{3} ^{0}$ & 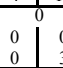 & 3 & 3 & 3 & Ap & & $\mathrm{h}$ & Paran mio \\
\hline Plantago urvillei Opiz & $21 \frac{1}{2}$ & : & 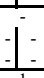 & & 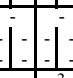 & : & & 1 & : & 1 & 1 & i. & : & : & : & & is & $122_{2}^{2}$ & 12 & $\left.\right|_{6} ^{5}$ & $\begin{array}{llll}4 & 1 \\
8 & 1 & \\
8\end{array}$ & 6 & ${ }^{21}$ & 33 & $N_{s s}$ & & h & Fest-Brom, Gali veri \\
\hline Poo angustifilia L. & 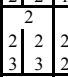 & $\frac{2}{3}-20$ & 年 & & 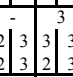 & 型 & & $2\left|\begin{array}{ll}1 \\
3 \\
3\end{array}\right| \begin{array}{ll}1 \\
2\end{array}$ & i & & 2 & 造 & 2 & $\left.\frac{1}{2}\right|_{3} ^{2}$ & 这党 & & $2=\frac{2}{3}$ & $\left|\begin{array}{l}3 \\
3\end{array}\right|^{3}$ & 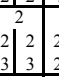 & $3_{2}^{2}$ & $\begin{array}{ll}437 \\
429 \\
69\end{array}$ & 29 & 130 & 289 & Ha & & gh & 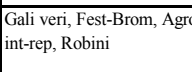 \\
\hline Poa bulbosa L. & : & & 1 & & 2 & & & : & $\therefore$ & & & -1 & 1 & 1 & 1 & & 1 & 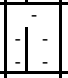 & 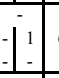 & $\left.\right|_{2} ^{16}$ & $\frac{10}{101}$ & 13 & 23 & 36 & Ha & & h & 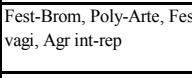 \\
\hline Poa compressa L. & : & 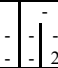 & 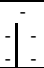 & & : & 1 & & : & $\because$ & 12 & $\begin{array}{lll} & 1 \\
3 & 0\end{array}$ & a & $1:$ & 1: & : : & & $a_{3}$ & : & : & $j_{6}^{1}$ & 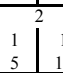 & 6 & ${ }^{11}$ & 24 & $\mathrm{Ha}$ & & ${ }_{\mathrm{h}}$ & 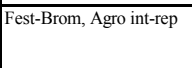 \\
\hline Doa pratensisis. $\mathrm{L}$. & : & & & & 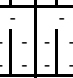 & & & . & 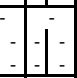 & & is & E & 1 & $1:$ & 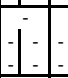 & & 1 & : & E & $\prod_{1}^{0}-2$ & 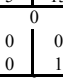 & 1 & 1 & 1 & Ha & & 8 & \\
\hline $\begin{array}{l}\text { Polygonatumim hirt tur } \\
\text { (Bosc ex Poir.) }\end{array}$ & 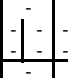 & 1 & 1 & & a: $: 1$ & : : & $: 1$ & $\therefore: 1$ & $1:$ & : & $1:$ & 1: & 1 & $1:$ & $:$ & & : : : & $1:$ & : & $\left|\begin{array}{lll}0 \\
1\end{array}\right|$ & 等 & 1 & 1 & 2 & $N_{s s}$ & & 8 & Pacr- Fase \\
\hline 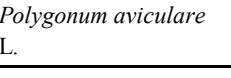 & is & : & I: & : & i: & A: & $\therefore$ & 1 & $\therefore$ & : & $=$ & 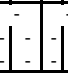 & : & : & : & & 1 & $1:$ & $A:$ & $\mid$\begin{tabular}{|l}
0 \\
1 \\
1
\end{tabular} & 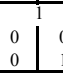 & \begin{tabular}{|l|l}
0 \\
\end{tabular} & 2 & 2 & $A_{p}$ & & $t$ & \\
\hline
\end{tabular}




\begin{tabular}{|c|c|c|c|c|c|c|c|c|c|c|c|c|c|c|c|c|c|c|c|c|c|c|c|c|c|c|c|c|}
\hline & & & & & & & & & & & & & & & & & & & & 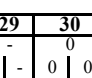 & $\begin{array}{lll}310 \\
0 & 010 & 0\end{array}$ & & & & & 36 & & 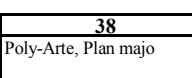 \\
\hline $\begin{array}{l}\text { Boreau } \\
\text { Polygonum bellardii }\end{array}$ & : & & : & -1 & & & 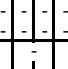 & 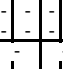 & -1 & & & & . & & : : & & & : & 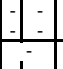 & $\begin{array}{lll}0 & 0 \\
0 & 1 \\
0 & 1\end{array}$ & $\begin{array}{lll}0 & 0 \\
0 & 0 & 1 \\
0 & 0 & 0\end{array}$ & \begin{tabular}{l|l}
0 & 3 \\
3 & 3 \\
3
\end{tabular} & $3^{3}$ & & Ap & & & Fest tagi, A Attemi \\
\hline All. s.str. & it & & & & & & & & & & & & 1 & & & & & & 1 & 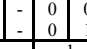 & & & & & $\left.\right|^{H a}$ & & & \\
\hline $\begin{array}{l}\text { Pellogon } \\
\text { Besser }\end{array}$ & $: 1$ & & & & & & & 1 & & & & & & & & & $: 1$ & & 1 & $\begin{array}{l}0 \\
0 \\
0\end{array}$ & 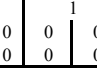 & $\begin{array}{l}0 \\
0 \\
\end{array}$ & 1 & 1 & $A_{p}$ & & t & 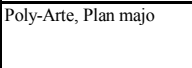 \\
\hline $\begin{array}{l}\text { Pologyonum patulum } \\
\text { M.Bieb. }\end{array}$ & 1 & & 1 & ii & $\therefore$ & & & 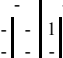 & & & & & 1 & & & & $\because:$ & it & it & $\left|\begin{array}{l}3 \\
2 \\
2\end{array}\right|$ & $\left.\begin{array}{l}1 \\
1 \\
1 \\
2\end{array}\right|^{2}$ & 1 & 8 & 9 & Ha & & t & \\
\hline Potentilla argentea $\mathrm{L}$. & 1 & & 1 & 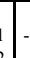 & 1 & & & & & & & :1: & & & & & $i:$ & i & ; & 15. & 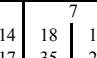 & \begin{tabular}{c|c}
16 & 25 \\
\end{tabular} & 69 & 102 & Ha & & $\mathrm{h}$ & 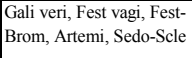 \\
\hline $\begin{array}{l}\text { Potentilla astracha- } \\
\text { nica Jacq. }\end{array}$ & 1 & & i: & $\therefore$ & it & 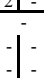 & 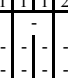 & 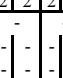 & & $\cdot 1$ & & $=1$ & 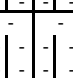 & I & 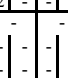 & i: & $\therefore$ & : & 1 & $\begin{array}{lll}1 & 1 \\
0 & 1 \\
0 & 1\end{array}$ & 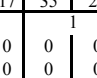 & \begin{tabular}{l|l}
0 & 1 \\
0 & 1
\end{tabular} & 1 & 1 & $N_{s}$ & & $\mathrm{~h}$ & Fest-Brom \\
\hline Potentilla heptaphylla & $1^{2}$ & & : & {$\left[\begin{array}{lll}1 & \end{array}\right.$} & $\therefore$ & & & & & & & & 1 & & & & & & 1 & 年: & $\frac{1}{1}: 1=$ & 2 & 1 & 2 & ${ }_{N s}$ & & $\mathrm{~h}$ & Fest-Brom \\
\hline \begin{tabular}{|l} 
Potentilla laciniosa \\
Kit. ex Nestl.
\end{tabular} & 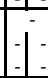 & & i & : & $\left|\begin{array}{ll}1 \\
1\end{array}\right|$ & & . & 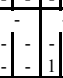 & 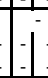 & & & 1 & 2 & 14 & & $2]$ & : : & 1 & : & \begin{tabular}{|ll}
8 \\
8 \\
4 \\
\end{tabular} & \begin{tabular}{|c|c|}
2 & 13 \\
1 & 13 \\
\end{tabular} & \begin{tabular}{l|l}
2 & 11 \\
1
\end{tabular} & ${ }_{16}^{16}$ & ${ }^{21}$ & $N_{s}$ & & ${ }^{\mathrm{h}}$ & Fest-Brom \\
\hline $\begin{array}{l}\text { Potentilla neglecta } \\
\text { Baumg. }\end{array}$ & 1 & iil & L & 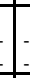 & il & 1 & & & & & & is & 1 & $1:$ & & & : & i & il: & $\begin{array}{l}3 \\
7]_{2}^{0} \\
2\end{array}$ & \begin{tabular}{|l|l|l|l|}
0 & 3 \\
2 & 7 & 1 \\
\end{tabular} & $\begin{array}{l}0 \\
2 \\
\end{array}$ & 13 & 13 & $H a$ & & ${ }^{\mathrm{h}}$ & \\
\hline \begin{tabular}{|l} 
Potentilla recta $\mathrm{L}$. \\
.
\end{tabular} & 1 & & $\mathrm{I}^{2}$ & i & i. & & & 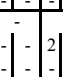 & & & & & 2. & & & & 2 & 1 & $1:=$ & $15: 1$ & 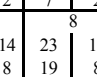 & 17 & 57 & 75 & ${ }_{\text {ss }}$ & & $\mathrm{h}$ & Fest-Broum \\
\hline $\begin{array}{l}\text { Prinusus divaricata } \\
\text { Lecte. }\end{array}$ & : & : & L & 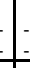 & $: 1$ & & & & i: & 1 & & $1:=1$ & : : & & -1 & : : & 1 & $: 1$ & : & $\%$ & $\begin{array}{lll}0 & 0 \\
2 & 0 & 0\end{array}$ & 0 & ${ }^{2}$ & ${ }^{4}$ & Ke & 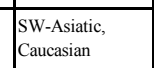 & ${ }_{\mathrm{nf}}$ & Robin \\
\hline Prunus domessica L. & 1 & & 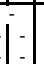 & 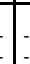 & . & & & 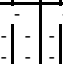 & . & & & & $\begin{array}{llll} & & & \end{array}$ & & & & 5 & & 5 & $\mid$ & $\begin{array}{lll}0 & 0.0 \\
0 & 0 & 0\end{array}$ & 0 & 3 & 3 & $E_{g}$ & 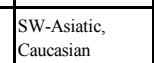 & $\mathrm{mf}$ & Robiin \\
\hline $\begin{array}{l}\text { Prunus cerasiffera } \\
\text { Ehkr. }\end{array}$ & $: 1$ & & 1 & $\therefore$ & $\mid: 1$ & il: & 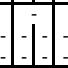 & : & 1 & & & : & : & $1:$ & 1 & : & : : & $: 1$ & $1:$ & il: & 年 & 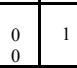 & 1 & 1 & ${ }_{\mathrm{Ke}}$ & 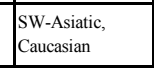 & ${ }_{\mathrm{nf}}$ & Robin \\
\hline $\begin{array}{l}\text { Prunum stepposa } \\
\text { Kotov }\end{array}$ & i & $\begin{array}{l}2 \\
3 \\
\end{array}$ & i & : & 1 & & 1 & 2 & : & & i: & $\mathrm{ij}_{2}$ & $1: 12$ & $a^{2} 2_{2}^{2}$ & it & A & $1 \frac{2}{2}$ & 1 & $\frac{1}{2}$ & {$\left[\begin{array}{l}2 \\
3\end{array}\right.$} & $\begin{array}{ll}6 \\
6\end{array}$ & 15 & ${ }_{33}$ & ${ }^{2} 2$ & ${ }_{N s}$ & & nf & Ramernum \\
\hline Pyrus communis L. & 1 & & & & $\therefore$ & & & i: & & & & & & & & & & & i i $i_{2}$ & $\begin{array}{lll}1 & 0 \\
0 & 0 \\
1 & 0 \\
4\end{array}$ & 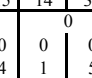 & 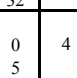 & ${ }^{5}$ & ${ }^{6}$ & Ae & & $\mathrm{mf}$ & Robin, [Quer-Fage] \\
\hline \begin{tabular}{|l|}
$\begin{array}{l}\text { Ranunculus } \\
\text { oxyspermus Willd. }\end{array}$ \\
\end{tabular} & : & & 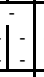 &. & & & . & & & & & & -1 & -1 & & -1 & & & : & $\begin{array}{ll}1 & 1 \\
0 & 0 \\
0\end{array}$ & Lis r r a & 1 & 1 & 1 & $\mathrm{Ns}_{\mathrm{s}}$ & & hg & Fats-Brom \\
\hline polvanthemos $\mathrm{L}$. & $: 1$ & & : & 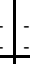 & $\therefore$ & 1 & : & i: & 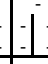 & & & $A:$ & : & $1: 1$ & $: 1$ & A: & $1:$ & $: 1$ & ii & 00 & 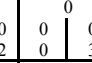 & \begin{tabular}{l|l}
0 & 2 \\
3 & \\
\end{tabular} & ${ }^{2}$ & 3 & $N_{s}$ & & hg & \\
\hline $\begin{array}{l}\text { Ramunculus scythicus } \\
\text { Klokov }\end{array}$ & - & & 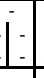 & 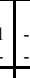 & .1 & & 2 & 2 & - & 1 & . & 2 & 21 & $\left|\frac{2}{2}\right|^{2}$ & 1 & $=1$ & $\therefore$ & 1 & 1 & $5 \begin{array}{l}5 \\
2 \\
2 \\
3 \\
3\end{array}$ & $9 \begin{array}{l}3 \\
4 \\
4\end{array}$ & $\begin{array}{c}15 \\
6 \\
\end{array}$ & 21 & ${ }^{37}$ & $n^{N s}$ & & $\mathrm{gh}^{\mathrm{gh}}$ & 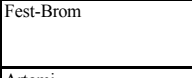 \\
\hline $\begin{array}{l}\text { Rapistrum perenne (L. } \\
\text { All. }\end{array}$ & : : & & 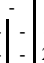 & i & 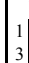 & & & is & & i & & & i: & i & 3 & 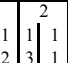 & & & j. & 年 & 2012 & 22 & 70 & 104 & $\mathrm{Ke}$ & Mecilier & $\mathrm{h}$ & Antemi \\
\hline Resedda lutea $\mathrm{L}$. & -1 & & & & & & 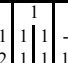 & ل & & & & & & 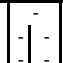 & & & & & i & $77^{5}$ & 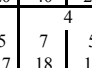 & 5 & 50 & 53 & Ke & Mecilier & & 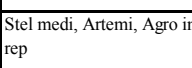 \\
\hline Rhamnus cathartica $\mathrm{L}$ & |: & & & 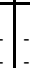 & & & & i & & & if & 1 & H & il & -1 & ilit & ii & 烈 & i: & $\left.a_{5}^{2}\right]_{9}^{2}$ & 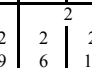 & 9 & 20 & 24 & $N_{s}$ & & nf & \\
\hline $\begin{array}{l}\text { Robinia pseudoacacia } \\
\text { L. }\end{array}$ & 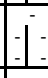 & & & & & & & & & & & & & & & & $i:$ & & i & $\left.{ }_{3}^{1}\right]_{3}^{2}$ & $\left|\frac{1}{4}\right|^{2}$ & $\begin{array}{c}4 \\
\end{array}$ & ${ }_{10}^{10}$ & 16 & Ke & North Ameriean & $\mathrm{mf}$ & Robin \\
\hline Rosa canina $\mathrm{L}$. & 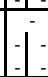 & & & & & & & & & & & & & & & & & & & & & 3 & ${ }^{6}$ & ${ }^{6}$ & $N_{s s}$ & & nf & \\
\hline
\end{tabular}




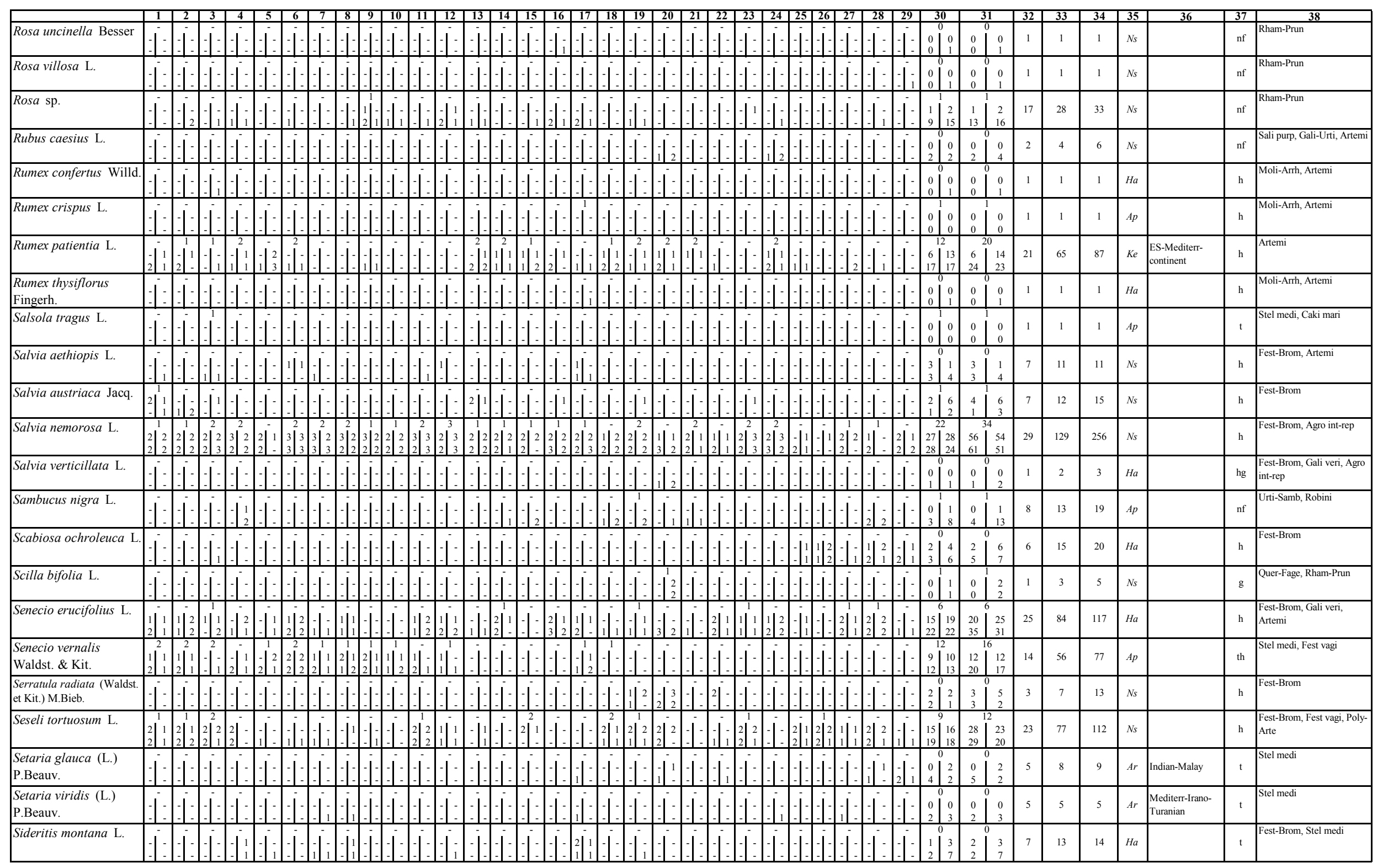




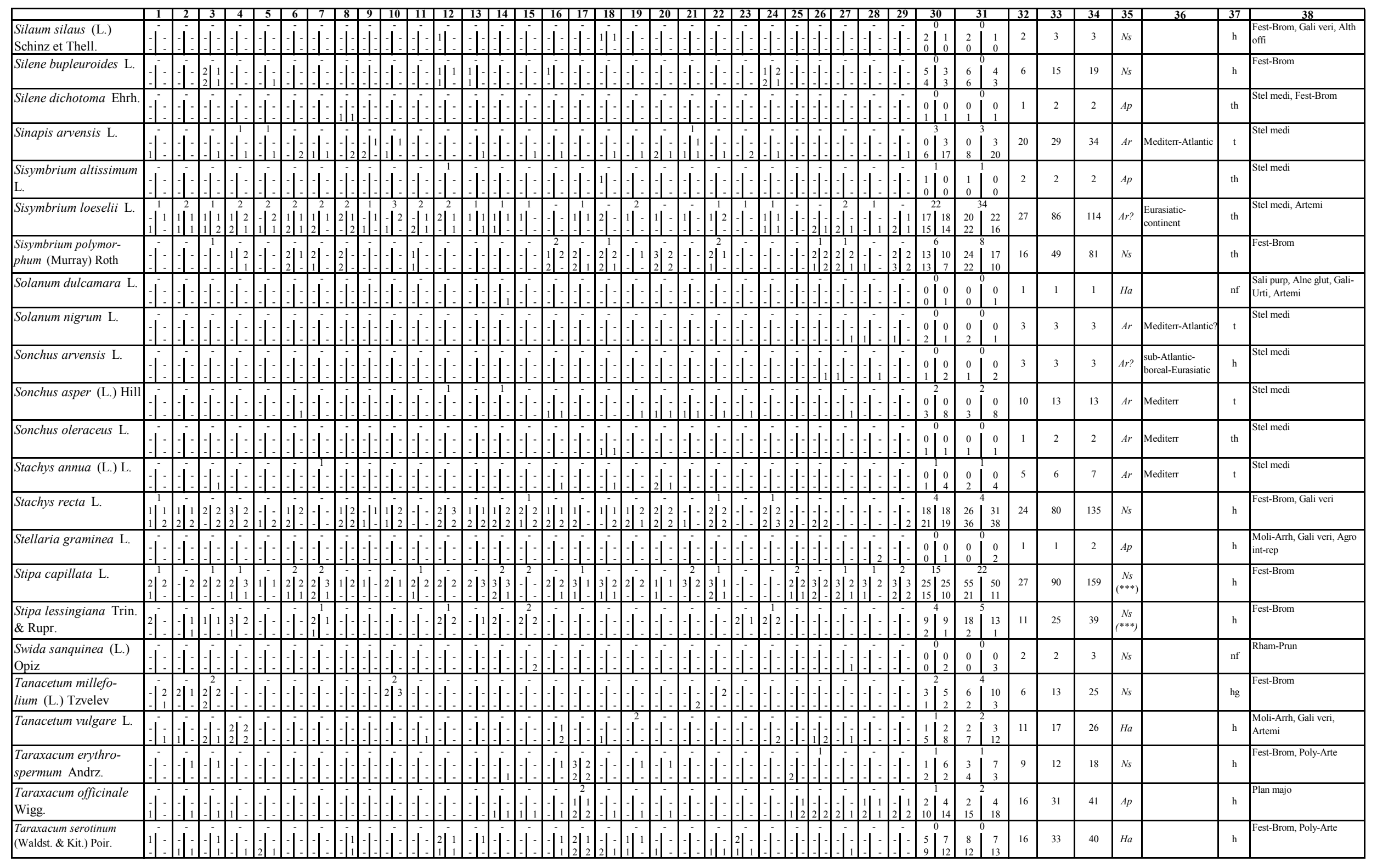




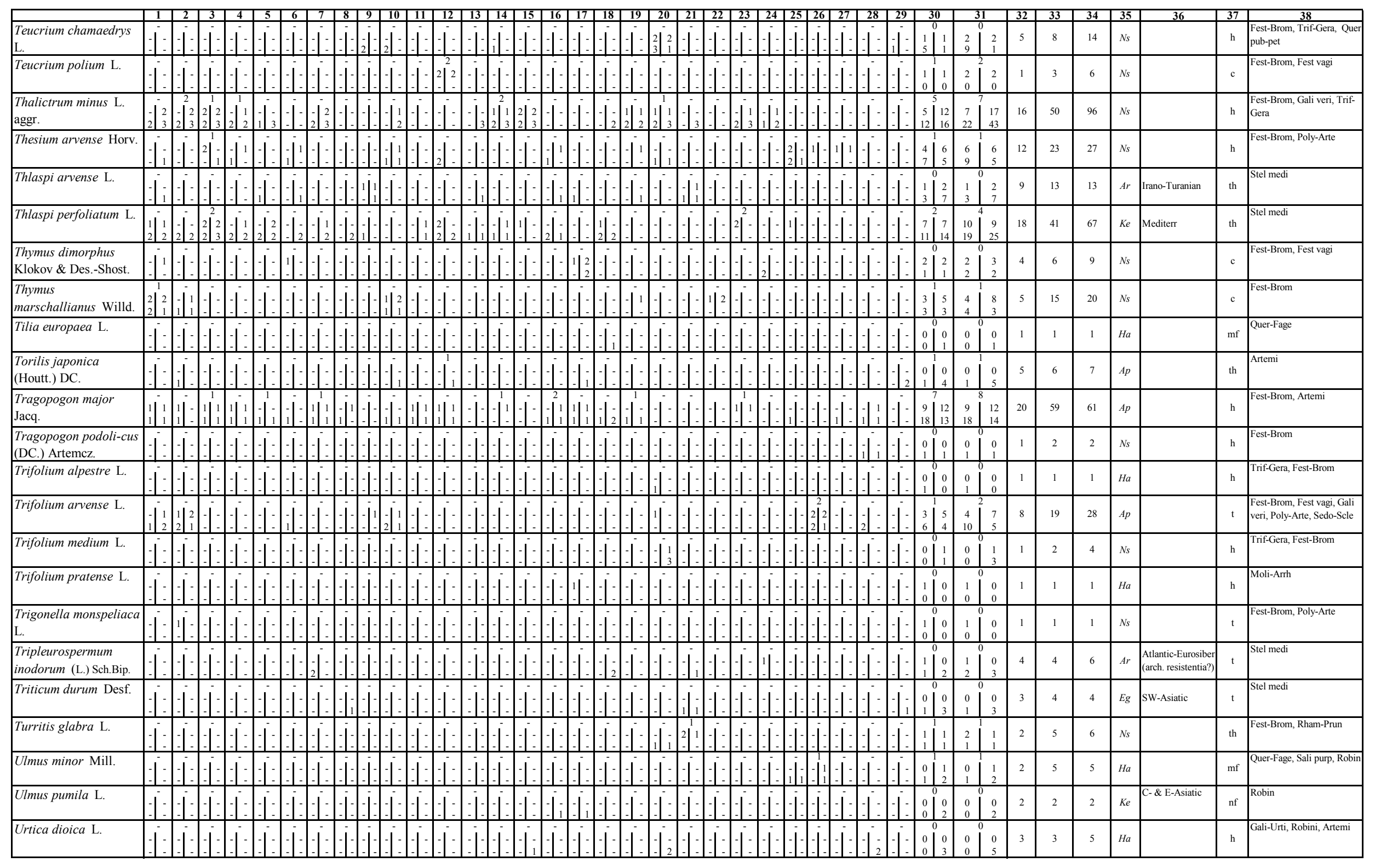




\begin{tabular}{|c|c|c|c|c|c|c|c|c|c|c|c|c|c|c|c|c|c|c|c|c|c|c|c|c|c|c|c|c|}
\hline Urtica urens L. & $1-1$ & & & .5 & & & & & & & & & & & & & & & & 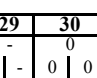 & & & 33 & & $\mid \frac{35}{45}$ & $\begin{array}{l}36 \\
\text { Metiter }\end{array}$ & & 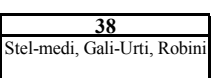 \\
\hline & [.] & & & & & & : & & & & & & & & & & & & $=1$ & $\left.=]_{0}^{0}\right]_{1}^{0}$ & $\begin{array}{lll} & 0 \\
1 & 0 & 0 \\
0\end{array}$ & & 1 & & & & & \\
\hline teriana tuber & & & & & & & 1 & & & & & & & & & & & & & $\left|\begin{array}{ll}0 \\
0 \\
0\end{array}\right|$ & $\because 1$ & 1 & 1 & 1 & $\mathrm{Ns}_{\mathrm{s}}$ & & $\mathrm{g}$ & 然 \\
\hline Valeriana officinalis $\mathrm{L}$ & |: 1 & 1 & $\therefore$ & - & & & $1: 1$ & & & & & & & & & & & & & 尚 & $\begin{array}{lll}2 & 3 \\
0 & 3\end{array}$ & ${ }^{3}$ & 6 & ${ }_{10}$ & $N_{s}$ & & 8 & 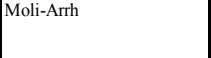 \\
\hline $\begin{array}{l}\begin{array}{l}\text { Valerianella carinata } \\
\text { Loisel. }\end{array} \\
\text { a }\end{array}$ & $:$ & 1!: & : & 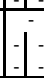 & & & 1 & ins & 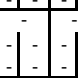 & & & & : & & & A & & & 1 & $\left(\begin{array}{lll}2 & 2 \\
2 & 1 \\
0 & 1 \\
0\end{array}\right.$ & 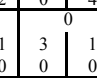 & 2 & 3 & 4 & Ha & & $\mathrm{t}$ & 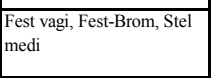 \\
\hline 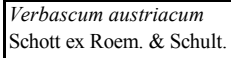 & 2 & : & | & 1 & & & 1 & $z_{2}-2$ & $=1$ & & & & 11. & & & : & !. & & 4 & 品 & 186 & ${ }_{18}$ & 42 & 60 & ${ }_{N s}$ & & $\mathrm{~h}$ & ment \\
\hline Tausch & :1! & 1 & It & J & & & $1: 1$ & 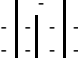 & & & & & & & & & & & H: & 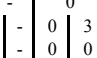 & $\mid \begin{array}{ll}0 & 0 \\
0 & 3 \\
0\end{array}$ & ${ }^{3}$ & ${ }^{3}$ & 3 & $N_{s}$ & & h & Ferbibiom \\
\hline & $2=2$ & & |. & t & & 1 & $1-1$ & & & & & & & & & & & & 2 & 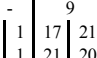 & $=2112$ & 26 & 88 & ${ }_{128}$ & Ha & & $\mathrm{h}$ & 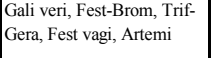 \\
\hline Verbascum phlomoides & ii & 1 & 1 & it & 1 & a & il & $\frac{1}{1} 1$ & & & & & : & : & & i & $: \mathrm{i}:$ & & $\because$ & $\mid \begin{array}{c}6 \\
0 . \\
0\end{array}$ & $\left.4\right|^{4} \mid \begin{array}{l}2 \\
11\end{array}$ & \begin{tabular}{c|c}
4 \\
$\begin{array}{c}1 \\
1\end{array}$
\end{tabular} & 30 & 33 & Ha & & $\mathrm{h}$ & Aremin \\
\hline $\begin{array}{l}\text { Verbascum phoeniceun } \\
\text { L. }\end{array}$ & $: 1_{2}^{2}$ & ii & 1 & 1 & T. & 1 & 1 & : & 1 & & & & $\begin{array}{l}1 \\
2 \\
-12 \\
2\end{array}$ & $i$ & & 2 & : & & 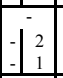 & \begin{tabular}{|l|l|l|l|l|}
2 \\
9 \\
9
\end{tabular} & \begin{tabular}{ll|l} 
& 3 \\
0 & 14 & 1 \\
8 & 14 \\
\end{tabular} & ${ }_{14}$ & ${ }_{35}$ & 57 & $N_{s}$ & & $\mathrm{~h}$ & Fest-Brom, 1 \\
\hline eronica arvensis $\mathrm{L}$. & j. & j & 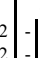 & . & 型 & & il & $-\left.i_{3}^{1}\right|^{2}$ & & & & & $\left.2^{2}\right|^{2}$ & i & & & & 1 & 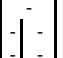 & {$\left[\begin{array}{lll}4 \\
3 \\
3\end{array}\right.$} & 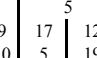 & 18 & 35 & 58 & ${ }_{A r}$ & 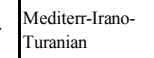 & th & 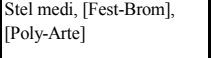 \\
\hline Veronica austriaca $\mathrm{L}$. & 1 & . & 1 & 1 & 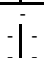 & & 1 & & . & & & & & & & & - & & | & $1^{2} 10$ & : & 13 & 18 & ${ }^{23}$ & $N_{s}$ & & 。 & Festriom \\
\hline Yeronical longifolia L. & 1 & : & 1 & 1 & : & 1 & 1 & : & : & & & & $\therefore$ & : : & & $A$ & & . & : & ilio & \begin{tabular}{l|l|l} 
& 0 & 0 \\
0 & 1 & 1 \\
1
\end{tabular} & 1 & ${ }^{2}$ & 2 & $N_{s}$ & & $\mathrm{~h}$ & Moli-Arith \\
\hline eronica polita $\mathrm{Fr}$. & 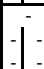 & i & $i$ & 1 & 1 & & 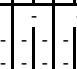 & : & $\%$ & & & & i): & : & -1 & 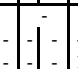 & i: & : & : & \% & $\begin{array}{llll}0 & 0 \\
0 & 0 & 0 & 0\end{array}$ & 2 & 2 & 2 & $A r$ & $\begin{array}{l}\text { Meditern-rano- } \\
\text { Turanianon }\end{array}$ & $\mathrm{t}$ & Sitel medi \\
\hline Veronica praceox All. & 1 & & 1 & 1 & i & & i & & & & & & 1 & : & & : & $: 1$ & & : & $\left|\begin{array}{l}7 \\
4\end{array}\right|_{1}^{5}$ & \begin{tabular}{|l|l|l}
9 & 9 \\
6 & 1 \\
\end{tabular} & 12 & 18 & ${ }^{26}$ & $H a$ & & th & 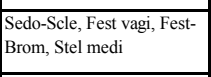 \\
\hline Veronica prostrata L. & 11 & 2 & 2 & 1 & 1 & & 1 & : & : & & & & $\therefore$ & i & : & : & : & : & : & $\left.\begin{array}{|l|l}4 \\
3 \\
3\end{array}\right]_{14}^{4}$ & $\begin{array}{ll}4 \\
4 \\
4\end{array}$ & ${ }^{4}$ & 13 & 19 & $N_{s}$ & & . & \\
\hline 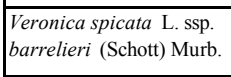 & $\mid \begin{array}{lll}2 \\
2\end{array}$ & 2 & & 1 & 1 & & 1 & . & & & & & 1 & 1 & & & & 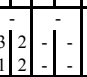 & 1 & $\left.\mid \begin{array}{ll}7 \\
5\end{array}\right]_{8}^{9}$ & $\begin{array}{lll} \\
9 \\
9\end{array}$ & ${ }^{10}$ & 35 & 56 & $s_{s}$ & & ${ }^{\mathrm{h}}$ & ert-Brom \\
\hline eronica spuria $\mathrm{L}$. & 1 & 1 & -1 & : & - & & 1 & : & 1 & & & & |' & 21 & $\mathrm{~T}$ & : & $\therefore$ & $1:$ & 1 & {$\left[\begin{array}{l}4 \\
3\end{array}\right]_{2}^{4}$} & $\begin{array}{lll}4 & 5 \\
4 & 0 \\
4 & 7 \\
3\end{array}$ & 5 & 13 & 19 & $N_{s}$ & & ${ }_{\mathrm{h}}$ & Fest-Brom, TrifGera \\
\hline Veronica teucrum L. & -1 & 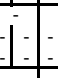 & & : & & 1 & i & . & & & & & 1 & & & & & & i & $\begin{array}{l}3 \\
4 \\
4\end{array}$ & \begin{tabular}{ll|l} 
& 4 \\
4 & 6 & 0 \\
\end{tabular} & ${ }^{10}$ & ${ }_{18}$ & 22 & $N_{s}$ & & $\mathrm{ch}$ & Fest-Brom, Moli-Arnh \\
\hline Veronica triphyllos L. & 1 & 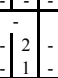 & 1 & 1 & . & & 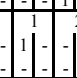 & : 1 & 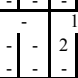 & & & $2=$ & 1 & I: & & : & & & : & {$\left[\begin{array}{l}6 \\
2\end{array}\right]_{1}^{3}$} & $\begin{array}{l}34 \\
1045 \\
3\end{array}$ & ${ }_{10}$ & 15 & 23 & Ar & 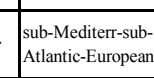 & $t$ & Sielinmed \\
\hline eronici verna L. & 步 & & & d. & & & 1 & & & & & & & & & & & & $1:$ & s. & \begin{tabular}{l|l}
810 \\
4
\end{tabular} & ${ }_{16}$ & 25 & 38 & Ha & & 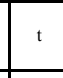 & $\begin{array}{l}\text { Fistr-Bom, Fe } \\
\text { Ante } \\
\end{array}$ \\
\hline Reicichardustiffolia & 1 & & & t & & & -1 & & & & & & & -1 & & & & & 1 & i. & \begin{tabular}{ll|l} 
& 0 \\
0 & 0 & 1 \\
1 & 1 & 1 \\
1
\end{tabular} & 1 & 3 & ${ }^{3}$ & Ke? & Mcaliter? & i & Sict meti \\
\hline Yicia lathrovid & 1 & & & & & & & & & & & & & & & & & & & 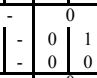 & $\begin{array}{llll}0 & 1 \\
0 & 0 & 0 \\
0\end{array}$ & 1 & 1 & 1 & $N_{s}$ & & th & Fats-Brom, \\
\hline Ficia sativa L. & 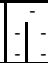 & : & & & & & 1 & & & & & & & & & & & & & 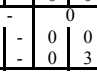 & \begin{tabular}{|l|l|l|l}
0 & 0 \\
0 & 0 & 0 \\
0 & 3 & 3 \\
\end{tabular} & \begin{tabular}{l|l}
0 \\
\end{tabular} & 3 & 3 & $E_{g}$ & Meditier (?) & 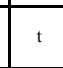 & \\
\hline
\end{tabular}




\begin{tabular}{|c|c|c|c|c|c|c|c|c|c|c|c|c|c|c|c|c|c|c|c|c|c|c|c|c|c|c|c|c|c|c|c|c|c|c|c|c|c|c|}
\hline & 1 & 2 & 3 & 4 & 5 & 6 & 7 & 8 & 49 & 10 & 11 & 12 & \begin{tabular}{|l|l|}
13 & 14 \\
\end{tabular} & \begin{tabular}{l|l|}
4 & 15 \\
& -15 \\
\end{tabular} & 16 & \begin{tabular}{|c|}
17 \\
\end{tabular} & 18 & 19 & 20 & \begin{tabular}{|l|}
21 \\
\end{tabular} & $|22|$ & $23 \mid$ & \begin{tabular}{|l|}
24 \\
\end{tabular} & \begin{tabular}{|l|l}
25 & 2 \\
\end{tabular} & 26 & 27 & 28 & 29 & 30 & & & 32 & 33 & 34 & 35 & 36 & 37 & \\
\hline Vicia tenuifolia Roth & $\left.\mid \begin{array}{l}1 \\
2\end{array}\right]:$ & $i$ & $\therefore$ & i & $\therefore$ & $-i_{2}^{2}$ & & & $-1-1$ & \begin{tabular}{ll|}
-1 & -1 \\
& -1
\end{tabular} & $-1-1$ & $\begin{array}{ll}-1 \\
-1 \\
-1\end{array}$ & 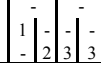 & \begin{tabular}{|c|c|c|}
- & - \\
& 2 \\
\end{tabular} & $\mid \begin{array}{cc}-1 & - \\
\end{array}$ & \begin{tabular}{|c|c|} 
& - \\
3 & -1 \\
\end{tabular} & $\begin{array}{ccc}-1 & - \\
2 & - & \end{array}$ & $-1-1$ & $\mid$\begin{tabular}{r|r|}
- & 1 \\
2 & 3 \\
\end{tabular} & $\mid$\begin{tabular}{l|l}
2 & 3 \\
3 & 3 \\
3 & 3
\end{tabular} & $-1-1$ & $-\begin{array}{c}-1 \\
-1\end{array}$ & \begin{tabular}{|l|l|l|}
1 & - \\
2 & - & \\
\end{tabular} & $\begin{array}{lll}1 & - \\
2 & - & -\end{array}$ & & $i-$ & \begin{tabular}{ll|}
-1 & -1 \\
& -1
\end{tabular} & $-1-1$ & \begin{tabular}{|c|c|}
5 & 1 \\
5 & 4 \\
11 & 8 \\
\end{tabular} & $\begin{array}{c}7 \\
23\end{array}$ & $\begin{array}{r}8 \\
20\end{array}$ & 14 & 29 & 60 & $\mathrm{Ha}$ & & gh & Trif-Gera \\
\hline $\begin{array}{l}\text { Vicia tetrasperma }(\text { L.) } \\
\text { Schrob. }\end{array}$ & 12 & $\therefore:$ & $1:$ & $1=$ & $\therefore:$ & $\therefore:$ & $1=$ & -1 & $\because-1$ & $1:-1$ & $-1:$ & $-1:$ & 10 & $-1:-1$ & $1:-1$ & $-1:$ & $\therefore:$ & $-1:$ & $\therefore:$ & $-1:$ & $1:$ & $\therefore:$ & $\because: 1$ & 1 & & $\therefore$ & $-1:$ & $\because:-1$ & \begin{tabular}{|l|l|}
0 & 0 \\
0 & 1
\end{tabular} & $\begin{array}{l}0 \\
0\end{array}$ & $\begin{array}{l}0 \\
2\end{array}$ & 1 & 1 & 2 & $A r$ & Mediterr & $\mathrm{t}$ & Stel medi \\
\hline Vicia villosa Roth & $\left.\begin{array}{ll}1 \\
2\end{array}\right]$ & 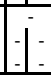 & -1. & 1 & 1. & $-i_{1}^{2}$ & $-1-$ & E & $-1-1$ & $-1-1$ & 1 & $-1-1$ & 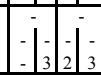 & \begin{tabular}{l|l|l|} 
& 2 & 2 \\
3 & 2 \\
\end{tabular} & \begin{tabular}{|c|c|}
- & 1 \\
2 & 3 \\
\end{tabular} & -1. & -1. & 1 & $-1-1$ & \begin{tabular}{|l|l|}
1 & 2 \\
2 & 2 \\
\end{tabular} & -1 & $-1-1$ & \begin{tabular}{|l|l|} 
& 1 \\
3 & 2 \\
\end{tabular} & - & . & $i$ & & $-\begin{array}{l}1 \\
2 \\
\end{array}$ & \begin{tabular}{|l|l|}
4 & 7 \\
9 & 9 \\
\end{tabular} & $\begin{array}{r}5 \\
20 \\
20\end{array}$ & $\begin{array}{l}11 \\
20\end{array}$ & 12 & 32 & 60 & $A r$ & Mediterr & th & Stel medi \\
\hline \begin{tabular}{|l|} 
Vinca herbacea \\
Waldst. et Kit.
\end{tabular} & 1 & \begin{tabular}{|l|l}
1 & 1 \\
2 & 2 \\
\end{tabular} & 1 & $1=$ & 1 & 1 & $\therefore$ i & -1 & $: 1$ & $1:$ & 10 & $: 1:$ & 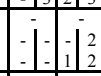 & \begin{tabular}{|l|l|l|}
2 & 2 \\
2 & 3 & 3 \\
2 & 2 \\
\end{tabular} & -1 & $: 1$ & $: 2$ & $2:-$ & \begin{tabular}{|l|l|} 
& 2 \\
& 2 \\
\end{tabular} & \begin{tabular}{|l|l|} 
& 2 \\
2 & 2 \\
\end{tabular} & -1 & -1 & $: 1:$ & -1 & . & 1 & 1 & $: 1=$ & \begin{tabular}{|l|l|}
3 & 5 \\
5 & 6 \\
\end{tabular} & $\begin{array}{r}7 \\
9 \\
\end{array}$ & $\begin{array}{l}10 \\
11 \\
\end{array}$ & 7 & 20 & 39 & Ns & & $\mathrm{g}$ & Fest-Brom, Trif-Gera \\
\hline $\begin{array}{l}\text { Vincetoxicum } \\
\text { hirundinaria Medik. }\end{array}$ & $1:$ & $1:$ & $2^{2}$ & $1=$ & 1 & 1 & -1 & $:-$ & $\therefore$ & 1 & & $\therefore:$ & -1. & $-1-1$ & -1 & $-1-1$ & $-1-1$ & 1 & \begin{tabular}{|l|l|}
-1 & 2 \\
2 & 2 \\
2 & 2 \\
\end{tabular} & $-1-1$ & 1 & 1 & $-1-1$ & -1 & 1 & -1 & 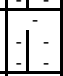 & 1 & \begin{tabular}{|l|l|} 
& 0 \\
0 & 2 \\
1 & 1 \\
\end{tabular} & $\begin{array}{l}0 \\
2 \\
\end{array}$ & $\begin{array}{l}4 \\
2 \\
\end{array}$ & 2 & 4 & 8 & Ns & & $\mathrm{g}$ & Trif-Gera, Fest-Brom \\
\hline Viola arvensis Murray & $i$ & 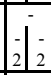 & $1-$ & $i$ & -1. & 1 & -1. & & \begin{tabular}{|c|} 
\\
$i$ \\
1 \\
\end{tabular} & $\begin{array}{ll} \\
1\end{array}$ & & $1-1$ & \begin{tabular}{ll|l} 
& -1 \\
\end{tabular} & $\begin{array}{r}-1 \\
1 \\
\end{array}$ & -1 & $\begin{array}{r}-i \\
1\end{array}$ & $-1-1$ & -1 & $\begin{array}{r}-1 \\
3\end{array}$ & \begin{tabular}{c|}
2 \\
-12 \\
2 \\
\end{tabular} & 1 & -1 & \begin{tabular}{|c|c|} 
& - \\
2 & - \\
\end{tabular} & -1 & & 1 & 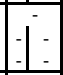 & 1 & \begin{tabular}{|l|l|} 
& 2 \\
1 & 1 \\
6 & 7 \\
\end{tabular} & $\begin{array}{r}1 \\
10 \\
\end{array}$ & $\begin{array}{c}2 \\
11 \\
\end{array}$ & 13 & 17 & 27 & $A r$ & anthropog? & th & Stel medi \\
\hline $\begin{array}{l}\text { Viola hirta } \mathrm{L} \text {. } \\
\text {. }\end{array}$ & -1 & $i=$ & $\bar{i}$ & -1 & $i_{i}$ & 1 & & & $-1-1$ & $-1:$ & $\because$ & $\mid \begin{array}{ll}- & - \\
1 & 1\end{array}$ & i & -1 & -1 & 1 & -12 & $\left.2\right|^{2}-1$ & il i & 1 & -1 & $\left|\begin{array}{lll}1 & 1 \\
2 & 2\end{array}\right|$ & $\because:-1$ & $\because$ & . & 1 & -1 & 1 & \begin{tabular}{|l|l|}
2 & 2 \\
& 4 \\
5 & 13
\end{tabular} & $\begin{array}{l}3 \\
6\end{array}$ & $\begin{array}{l}4 \\
18\end{array}$ & 13 & 26 & 34 & Ns & & $\mathrm{g}$ & Trif-Gera, Fest-Brom \\
\hline \begin{tabular}{|l} 
Viola kitaibeliana \\
Schult.
\end{tabular} & \begin{tabular}{|l|l} 
& 1 \\
2 & 2 \\
2 & 1 \\
2
\end{tabular} & $\left.\begin{array}{ll}2 \\
2 \\
2\end{array}\right|_{1} ^{2}$ & \begin{tabular}{|c|c|c|}
2 & 2 \\
2 & 2 \\
2 & 2
\end{tabular} & & \begin{tabular}{|l|l|l} 
& 1 \\
2 & 2 \\
2 & 1
\end{tabular} & \begin{tabular}{|l|l|l|} 
& 2 \\
2 & 1 \\
2 & 1 \\
2 &
\end{tabular} & $\mid$\begin{tabular}{l|l}
2 & 1 \\
2 & 1
\end{tabular} & & $\left|\begin{array}{cc}2 & 2 \\
2 & 2 \\
1\end{array}\right|$ & 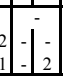 & \begin{tabular}{|l|l|l|l|l|l|l|l}
2 & 2 \\
2 & 2
\end{tabular} & $\mid \begin{array}{lll}2 & 1 \\
2 & 1\end{array}$ & $\begin{array}{l}2 \\
1\end{array} \mid$ & 2 & \begin{tabular}{|l|l|l}
2 & 2 \\
2 & 2
\end{tabular} & & $\left|\begin{array}{l}2 \\
1\end{array}\right|$ & 2 & \begin{tabular}{|}
2 \\
1
\end{tabular} \mid & il & 1 & 2 & \begin{tabular}{|l|l|l|} 
& 2 \\
2 & 2 \\
3 & 2 \\
\end{tabular} & 2 & & 1 & 1 & $1-$ & \begin{tabular}{|l|l|}
20 & 16 \\
17 & 14 \\
\end{tabular} & \begin{tabular}{l|l}
6 & 39 \\
4 & 30 \\
\end{tabular} & $\begin{array}{l}27 \\
20\end{array}$ & 23 & 79 & 137 & Ha & & $\mathrm{t}$ & Fest-Brom, Poly-Arte \\
\hline \begin{tabular}{|l|} 
Xanthium albinum \\
(Widder) H.Scholz \\
\end{tabular} & 1 & 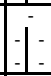 & $i$ & il & $i$ & 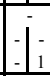 & 1 & : & il: & il: & $\begin{array}{lll}1 & 1 \\
\end{array}$ & -1 & -1 & $i$ & 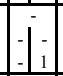 & \begin{tabular}{|c|c|} 
& - \\
2 & 1 \\
\end{tabular} & 1 & -1 & 1 & 1 & 1 & -1 & 1 & iَ & 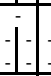 & 1 & 1 & -1 & \begin{tabular}{|l|l|} 
& 0 \\
0 & 0 \\
5 & 5 \\
5
\end{tabular} & $\begin{array}{l}0 \\
6 \\
\end{array}$ & $\begin{array}{l}0 \\
5 \\
\end{array}$ & 7 & 10 & 11 & $\mathrm{Ke}$ & North American & $\mathrm{t}$ & Stel medi, [Bident] \\
\hline $\begin{array}{l}\text { Xeranthemuт апnиит } \\
\text { L. } \\
\end{array}$ & $i$ & il & i & $\begin{array}{l}1 \\
1\end{array}$ & 1 & $i$ & $\begin{array}{ll}-1 \\
3\end{array}$ & i. & i & : & \begin{tabular}{|l|l}
1 & 1 \\
1 & 1 \\
1 & -
\end{tabular} & 1 & -1 & 1 & $-1-1$ & \begin{tabular}{l|l|} 
& 1 \\
1 & 2 \\
\end{tabular} & $-1-1$ & $\therefore 1$ & il: & $i$ & 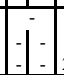 & i. & 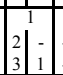 & -1. & i. & $\therefore$ & 1 & $1-$ & \begin{tabular}{|l|l|l|} 
& 2 \\
10 & 5 \\
\end{tabular} & $\begin{array}{c}6 \\
15\end{array}$ & $\begin{array}{l}2 \\
7\end{array}$ & 10 & 23 & 32 & $\mathrm{Ha}$ & & $\mathrm{t}$ & Fest-Brom, Poly-Arte \\
\hline The number of $\mathrm{T}$ species & 56 & 61 & \begin{tabular}{|l|}
-1 \\
70 \\
\end{tabular} & $\frac{112}{43}$ & $\begin{array}{l}-1- \\
27 \\
\end{array}$ & \begin{tabular}{|l|l|}
11 \\
41 \\
\end{tabular} & 40 & $\frac{1}{29}$ & 39 & \begin{tabular}{|l|}
-1 \\
30 \\
\end{tabular} & 35 & 39 & \begin{tabular}{|l|l|}
27 & 38 \\
\end{tabular} & 43 & 20 & \begin{tabular}{|l|}
12 \\
30 \\
\end{tabular} & \begin{tabular}{|l|}
-1 \\
43 \\
\end{tabular} & \begin{tabular}{|l|}
-1 \\
65 \\
\end{tabular} & 23 & \begin{tabular}{|l|}
-1 \\
49 \\
\end{tabular} & $\frac{1}{43}$ & 41 & 34 & \begin{tabular}{|l|l|}
16 & 3 \\
16 & \\
\end{tabular} & 31 & $\begin{aligned} 1-1 \\
27 \\
\end{aligned}$ & $\frac{1}{22}$ & 18 & & & & & & & & & & \\
\hline The number of Ss species & 75 & 72 & 71 & \begin{tabular}{|l|}
66 \\
\end{tabular} & 35 & 55 & \begin{tabular}{|l|}
60 \\
\end{tabular} & 44 & \begin{tabular}{|l|l|}
4 & 46 \\
\end{tabular} & \begin{tabular}{|l|}
48 \\
\end{tabular} & 53 & 59 & \begin{tabular}{|l|l|}
57 & 63 \\
\end{tabular} & 72 & \begin{tabular}{|l|}
44 \\
\end{tabular} & \begin{tabular}{|l|}
77 \\
\end{tabular} & \begin{tabular}{|l|l|}
76 \\
\end{tabular} & \begin{tabular}{|l|}
66 \\
\end{tabular} & 73 & \begin{tabular}{|l|}
56 \\
\end{tabular} & \begin{tabular}{|l|}
47 \\
\end{tabular} & 47 & \begin{tabular}{|l|l}
58 \\
\end{tabular} & \begin{tabular}{|l|l}
34 & 5 \\
\end{tabular} & \begin{tabular}{l|l}
50 & -1
\end{tabular} & 30 & 42 & 28 & & & & & & & & & & \\
\hline The number of Sn species & 100 & 101 & 104 & \begin{tabular}{|l|}
86 \\
\end{tabular} & \begin{tabular}{|l|}
56 \\
\end{tabular} & \begin{tabular}{|l|}
57 \\
\end{tabular} & \begin{tabular}{|l|}
66 \\
\end{tabular} & 59 & 51 & 64 & \begin{tabular}{|l|}
69 \\
\end{tabular} & 65 & \begin{tabular}{|l|l|}
64 & 66 \\
\end{tabular} & \begin{tabular}{|l|}
69 \\
\end{tabular} & \begin{tabular}{l|l}
80 \\
\end{tabular} & 68 & \begin{tabular}{|l|}
67 \\
\end{tabular} & \begin{tabular}{|l|}
78 \\
\end{tabular} & 81 & 63 & \begin{tabular}{|l|}
62 \\
\end{tabular} & 58 & \begin{tabular}{l|l}
54 \\
\end{tabular} & \begin{tabular}{|l|l}
41 & 4 \\
\end{tabular} & 48 & \begin{tabular}{|l|}
39 \\
\end{tabular} & 47 & \begin{tabular}{|l|}
51 \\
\end{tabular} & & & & & & & & & & \\
\hline The number of Bs species & \begin{tabular}{|l|l|}
104 \\
\end{tabular} & 107 & 80 & 93 & 74 & 85 & \begin{tabular}{|l|}
99 \\
\end{tabular} & 46 & \begin{tabular}{|l|l|}
568 \\
\end{tabular} & 78 & 59 & 59 & \begin{tabular}{|l|l|}
61 & 80 \\
\end{tabular} & \begin{tabular}{|l|l|}
0 & 68 \\
\end{tabular} & \begin{tabular}{|c|}
69 \\
\end{tabular} & 118 & \begin{tabular}{|l|}
85 \\
\end{tabular} & \begin{tabular}{|l|l|}
49 \\
\end{tabular} & 110 & \begin{tabular}{|l|}
57 \\
\end{tabular} & 54 & \begin{tabular}{|l|l|}
65 \\
\end{tabular} & \begin{tabular}{|l|l|}
82 \\
\end{tabular} & \begin{tabular}{|l|l}
57 & 5 \\
\end{tabular} & \begin{tabular}{l|l}
58 & -1 \\
\end{tabular} & \begin{tabular}{|l|}
64 \\
\end{tabular} & 67 & 73 & & & & & & & & & & \\
\hline The number of $\mathrm{Bn}$ species & \begin{tabular}{|l|}
104 \\
\end{tabular} & 112 & 103 & \begin{tabular}{|l|}
98 \\
\end{tabular} & 78 & \begin{tabular}{|l|}
83 \\
\end{tabular} & \begin{tabular}{|l|}
80 \\
\end{tabular} & 60 & 55 & 69 & 64 & 65 & \begin{tabular}{|l|l|}
72 & 95 \\
\end{tabular} & \begin{tabular}{|l|l|}
5 & 94 \\
\end{tabular} & \begin{tabular}{|l|}
79 \\
\end{tabular} & \begin{tabular}{|l|}
85 \\
\end{tabular} & \begin{tabular}{|l|}
105 \\
\end{tabular} & \begin{tabular}{|l|}
84 \\
\end{tabular} & \begin{tabular}{|l|}
100 \\
\end{tabular} & \begin{tabular}{|l|}
54 \\
\end{tabular} & \begin{tabular}{|l|}
59 \\
\end{tabular} & \begin{tabular}{|l|}
74 \\
\end{tabular} & \begin{tabular}{|l|}
73 \\
\end{tabular} & \begin{tabular}{|l|l}
53 & 6 \\
\end{tabular} & \begin{tabular}{l|l}
68 & -10 \\
\end{tabular} & \begin{tabular}{|l|}
79 \\
\end{tabular} & 74 & 73 & & & & & & & & & & \\
\hline The total number of species & 162 & 169 & 163 & 150 & 116 & 118 & 136 & 91 & \begin{tabular}{|l|l|} 
& 97 \\
\end{tabular} & 115 & 105 & 122 & \begin{tabular}{|l|l|}
122 & 147 \\
\end{tabular} & \begin{tabular}{|l|l|}
7 & 133 \\
\end{tabular} & 126 & 150 & \begin{tabular}{|l|}
165 \\
\end{tabular} & 134 & 171 & 101 & 109 & \begin{tabular}{|l|}
114 \\
\end{tabular} & \begin{tabular}{|l|}
116 \\
\end{tabular} & \begin{tabular}{|l|l|}
89 & 9 \\
\end{tabular} & \begin{tabular}{l|l}
98 & -1 \\
\end{tabular} & 110 & 106 & 103 & & & & & & & & & & \\
\hline $\begin{array}{l}\text { Other kurgans from this } \\
\text { zone: }\end{array}$ & & & & & & & & & & & & & & & & & & & & & & & & & & & & & & & & & & & & & & \\
\hline Allium guttatum Steven & & & & & & & & & & & & & & & & & & & & & & & & & & & & & & & & & & & & & & \\
\hline
\end{tabular}

